\title{
WestVirginiaUniversity
}

THE RESEARCH REPOSITORY @ WVU

Graduate Theses, Dissertations, and Problem Reports

2020

\section{Application of Decline Curve Analysis to Unconventional Reservoirs}

\author{
Majed Hisham Kabbani \\ mhk0007@mix.wvu.edu
}

Follow this and additional works at: https://researchrepository.wvu.edu/etd

Part of the Other Engineering Commons

\section{Recommended Citation}

Kabbani, Majed Hisham, "Application of Decline Curve Analysis to Unconventional Reservoirs" (2020). Graduate Theses, Dissertations, and Problem Reports. 7718.

https://researchrepository.wvu.edu/etd/7718

This Problem/Project Report is protected by copyright and/or related rights. It has been brought to you by the The Research Repository @WVU with permission from the rights-holder(s). You are free to use this Problem/Project Report in any way that is permitted by the copyright and related rights legislation that applies to your use. For other uses you must obtain permission from the rights-holder(s) directly, unless additional rights are indicated by a Creative Commons license in the record and/ or on the work itself. This Problem/Project Report has been accepted for inclusion in WVU Graduate Theses, Dissertations, and Problem Reports collection by an authorized administrator of The Research Repository @ WVU. For more information, please contact researchrepository@mail.wvu.edu. 


\title{
Application of Decline Curve Analysis to Unconventional Reservoirs
}

\section{Majed H. Kabbani}

\author{
Report submitted to the \\ Benjamin M. Statler College of Engineering and Mineral Resources at \\ West Virginia University \\ in partial fulfillment of the requirements for the degree \\ of \\ Master of Science in \\ Petroleum and Natural Gas Engineering
}

Kashy Aminian, Chair Ph.D.

Samuel Ameri, Professor

H. Ilkin Bilgesu, Ph.D.

Department of Petroleum and Natural Gas Engineering,

Morgantown, West Virginia

2020

Keywords: Decline Curve Analysis

Copyright 2020 Majed H. Kabban 


\title{
ABSTRACT \\ Application of Decline Curve Analysis to Unconventional Reservoirs
}

\author{
Majed H. Kabbani
}

Supply and demand are the main key factors for oil and gas industry. Moreover, natural gas was not important until early $18^{\text {th }}$ century after the World War II. The first shale gas well was successfully drilled $27 \mathrm{ft}$ deep in 1821. The well was drilled in the Devonian Dunkirk shale. Later in the 1980s producers began investigating into the more difficult tight gas sands in order to keep up with the growing market. A decade later in 1990s investors were looking at coalbed methane. Today, as demand is increasing globally, the oil and gas industry is moving toward shale.

Decline-curve analysis (DCA), is considered one of the most commonly used approaches to estimate the decline production rate and forecast future production rate for oil and gas wells. Accurate production forecast is very crucial for reserve estimation and development planning. However, shale production analysis utilizing traditional decline curve methods will not have an accurate production profile due to the nature of the reservoir properties and production behavior.

In this report, a comparison of new empirical equations has been studied specifically for shale production decline analysis. The recently developed methods are the Power Law Exponential Decline (PLE), Stretched Exponential Decline Model (SEPD) and Duong Model. The new decline curve approaches can predict future production rates for shale gas wells under transient flow. Duong model is considered simple and practical to use for predicting future forecast. Therefore, decline analysis was performed with Duong's method using actual production data for five (5) Marcellus Shale wells and the results were compared accordingly. 


\section{ACKNOWLEDGMENT}

I would like to express my appreciation to my research advisor Dr. Kashy Aminian for his support and guidance during my graduate program. His support, help and professional assistance made me completed my studies, which concluded in the achievement of M.S degree.

My Sincere appreciation and thanks to Professor Sam Ameri, Department Chair, for his continues support, motivation and guidance throughout my studies at West Virginia University. I would like also to express my deepest appreciation to the professors of the Petroleum and Natural Gas Engineering Department for the knowledge and experience provided to me.

Finally, my sincere gratitude and thanks to my family and friends for their continues support, love and efforts they have given to me. A special appreciation to Saudi Aramco for sponsoring my graduate study and supporting me throughout the program. 


\section{Table of Contents}

Abstract

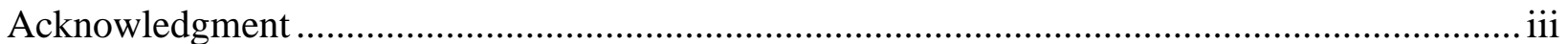

Table of Content …............................................................................................................ iv

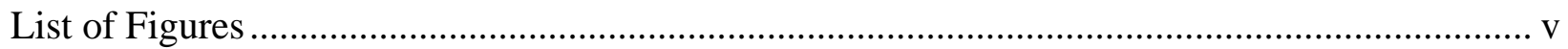

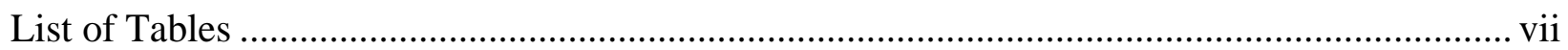

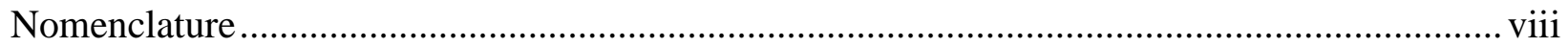

Chapter 1: Intoduction and Problem Statement ............................................................... 1

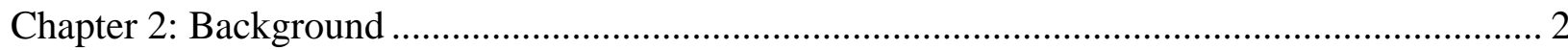

$2.1 \quad$ ARP's Model.....................................................................

2.2 Power Law Exponential Decline (PLE) ......................................4

2.3 Stretched Exponential Decline Model (SEPD) .................................................5

2.4 Duong Model.......................................................6

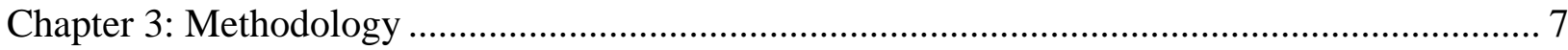

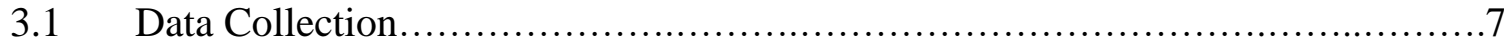

3.2 Data Analysis............................................................

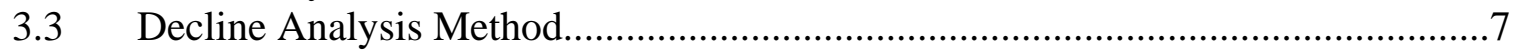

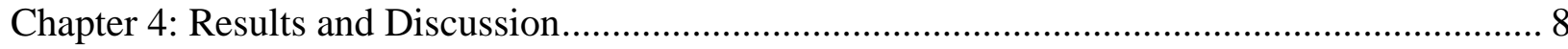

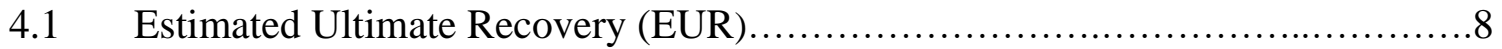

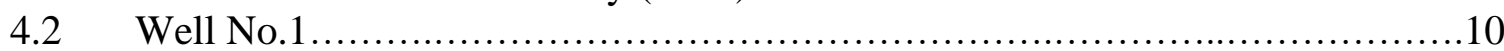

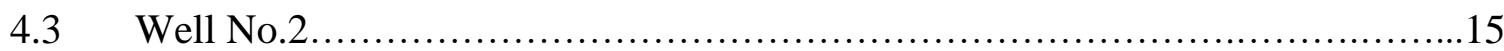

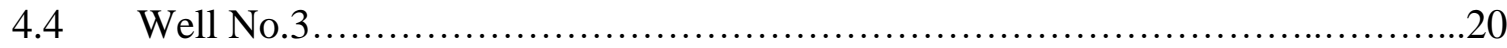

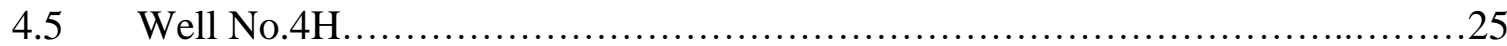

4.6 Well No.6H...................................................................

4.7 ARP's Model Analysis Well No.1 …........................................ 35

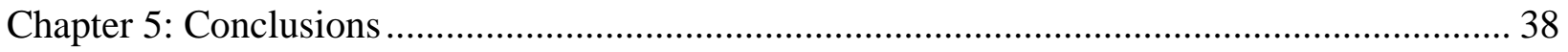

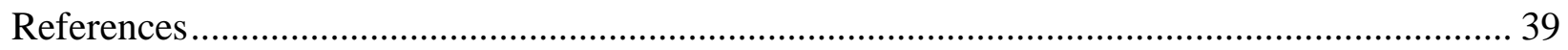

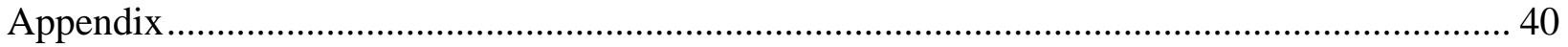




\section{List of Figures}

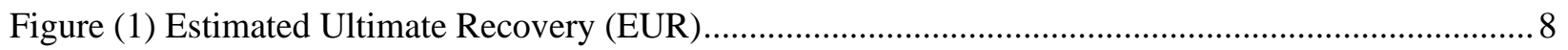

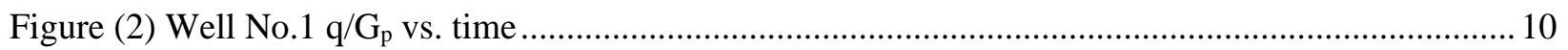

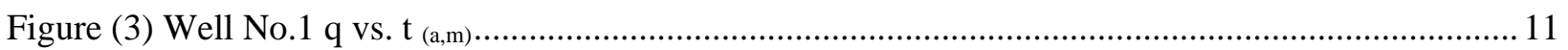

Figure (4) History Matching Well No.1 Gas Flow Rates with Duong Model .......................................... 13

Figure (5) Comparison of the Predicted and Actual Gas Flow Rates for Well No.1 ................................ 13

Figure (6) Predicted Gas Flow Rates for Well No.1 by Duong Model..................................................... 14

Figure (7) Comparison of Well No.1 Cumulative Production and Duong Model .................................... 14

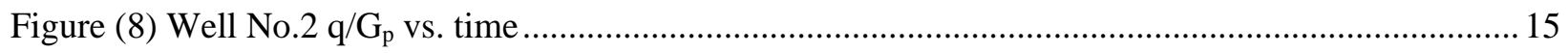

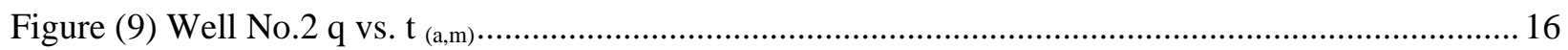

Figure (10) History Matching Well No.2 Gas Flow Rates with Duong Model ........................................ 18

Figure (11) Comparison of the Predicted and Actual Gas Flow Rates for Well No.2 ............................. 18

Figure (12) Predicted Gas Flow Rates for Well No.2 by Duong Model.................................................. 19

Figure (13) Comparison of Well No.2 Cumulative Production and Duong Model .................................. 19

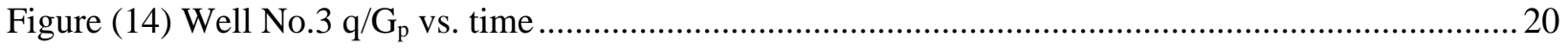

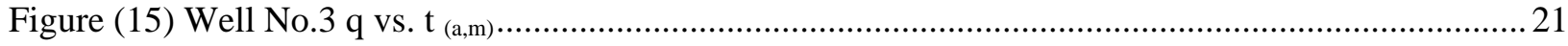

Figure (16) History Matching Well No.3 Gas Flow Rates with Duong Model ...................................... 23

Figure (17) Comparison of the Predicted and Actual Gas Flow Rates for Well No.3 …..........................23

Figure (18) Predicted Gas Flow Rates for Well No.3 by Duong Model...................................................24

Figure (19) Comparison of Well No.3 Cumulative Production and Duong Model ................................... 24

Figure (20) Well No.4H q/G

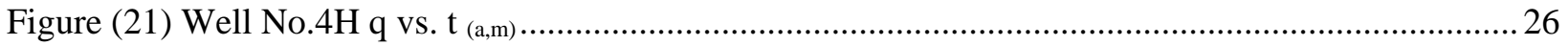

Figure (22) History Matching Well No.4H Gas Flow Rates with Duong Model .................................... 28

Figure (23) Comparison of the Predicted and Actual Gas Flow Rates for Well No.4H ......................... 28

Figure (24) Predicted Gas Flow Rates for Well No.4H by Duong Model ...............................................29

Figure (25) Comparison of Well No.4H Cumulative Production and Duong Model ...............................29

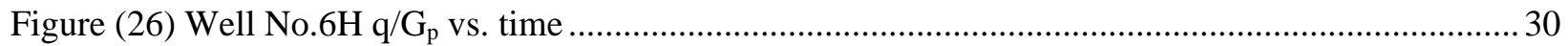

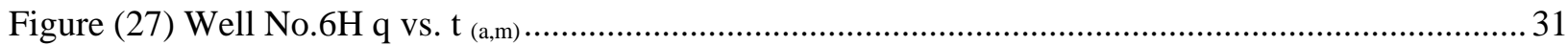

Figure (28) History Matching Well No.6H Gas Flow Rates with Duong Model .................................... 33

Figure (29) Comparison of the Predicted and Actual Gas Flow Rates for Well No.6H .......................... 33

Figure (30) Predicted Gas Flow Rates for Well No.6H by Duong Model .................................................. 34

Figure (31) Comparison of Well No.6H Cumulative Production and Duong Model ................................. 34 


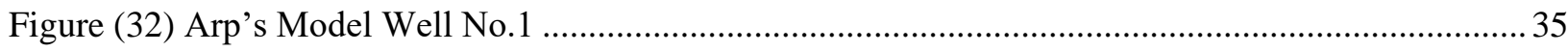

Figure (33) History Matching: Well No. 1 (ARP's Model vs. Duong Models) ....................................... 36

Figure (34) Comparison of the Predicted Gas Flow Rates for Well No.1 by Arps's and Duong Models .. 37 


\section{List of Tables}

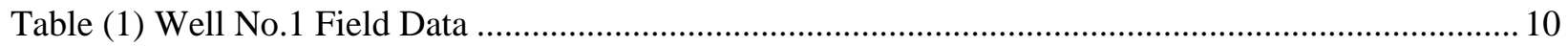

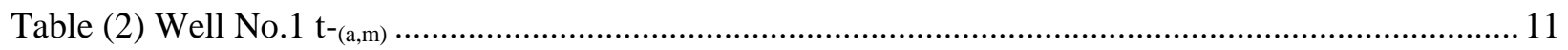

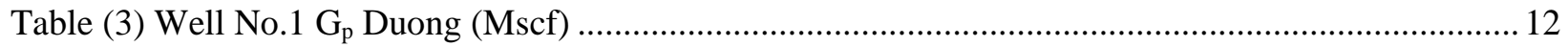

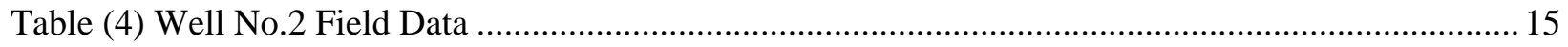

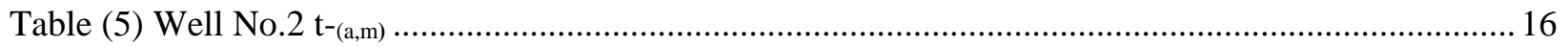

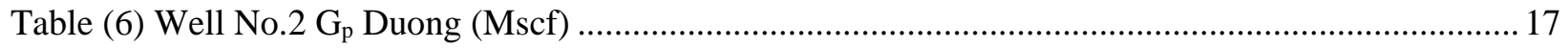

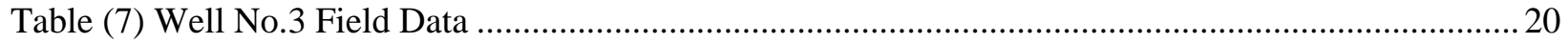

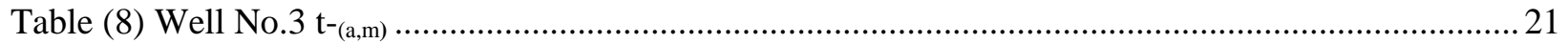

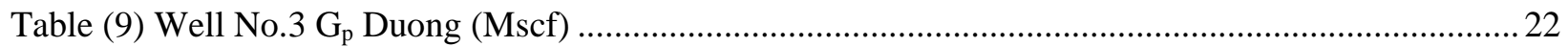

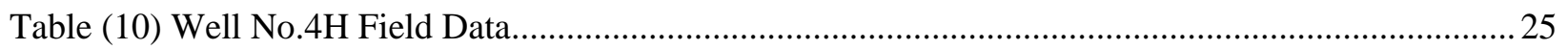

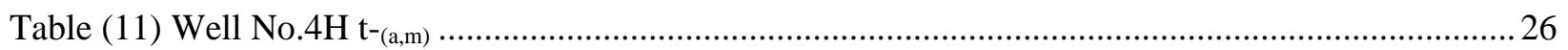

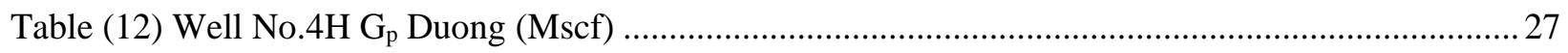

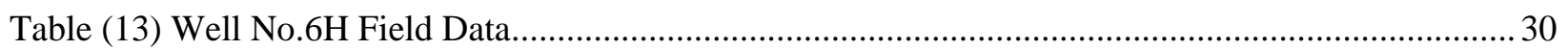

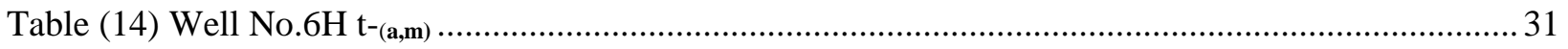

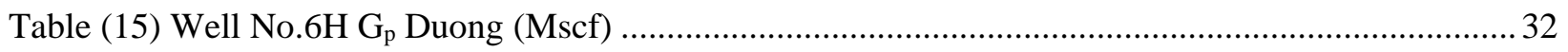




\section{Nomenclature}

EUR: Estimated Ultimate Recovery

DCA: Decline Curve Analysis

BDF: Boundary Dominated Flow

PLE: Power Law Exponential Model

SEPD: Stretched Exponential Production Decline Model

$\mathrm{di}=$ Loss Ratio, $1 /$ time

$\mathrm{qi}=$ Initial flow rate, volume/time

$\mathrm{n}=$ Derivative of Loss Ratio, dimensionless

$\mathrm{D} \infty=$ Loss ratio at $(\mathrm{t}=\infty), 1 /$ time

$\mathrm{D} 1=$ Loss ratio at $(\mathrm{t}=1), 1 /$ time

$\mathrm{q}=$ Flow rate, volume/time

$\mathrm{t}(\mathrm{a}, \mathrm{m})=$ Duong time function, dimensionless

$\mathrm{t}=$ time, years 


\section{Chapter 1: Introduction and Problem Statement}

Oil and gas companies were not interested in the investment of gas shale reservoirs since that it was not considered economical. However, due to the current advancement in technologies and the increasing of gas prices, investors are exploring all feasible options to recover and produce the gas that was once thought impossible to produce. Shale gas is considered now a key element of the energy sources in the United States. Shales have a significant amount of organic matter (kerogen) and serve as reservoir rock and source rock (El Sgher et al. 2018). The estimated consumption of natural gas in the U.S. is about 27 million cubic feet (MMcf) annually in 2017. Moreover, Natural gas has been the largest source of electrical generation since July 2015.

Drilling of a new well can be achieved vertically or horizontally. However, horizontal wells are more cost effective for commercial production in unconventional reservoirs (Shale). Production forecasts utilizing analytical and empirical approaches are not well established for horizontal wells with multi-stage hydraulic fractures due to limited field experience. Moreover, accurate production forecast is very crucial for reserve estimation and development planning. In additional, the production rate from unconventional reservoirs declines more rapidly than conventional reservoirs (El Sgher et al. 2018). There are many techniques for calculating and estimating shale gas reserves and performing production analysis. The most common approach to estimate the decline production rate and predict future production rate for oil and gas wells is Decline-curve analysis (DCA).

Arps' Decline Analysis (1945) can predict future production rates for conventional oil and gas wells that produce under boundary dominated flow (BDF). However, shale production analysis utilizing traditional decline curve methods will not have an accurate production profile due to the nature of the reservoir properties and production behavior. Therefore, the conventional DCA approach cannot be used to estimate production data from unconventional reservoirs. Recently, new empirical equations have been developed specifically for shale production decline analysis. These methods are the Power Law Exponential Decline (PLE), Stretched Exponential Decline Model (SEPD) and Duong Model. The new decline curve approaches can predict future production rates for shale gas wells under transient flow. 


\section{Chapter 2: Background}

Decline-curve analysis (DCA), is considered one of the most common technique to estimate the decline production rate and predict future production rate for oil and gas wells. Moreover, conventional oil and gas wells can be estimated with traditional Arps' Decline Analysis equation (1945) that typically produce under boundary dominated flow. However, new empirical equations have been developed specifically for shale production decline analysis. The models will be discussed in more details below.

\subsection{Arp's Models}

The first forecasting approach and most common traditional techniques was proposed by J.J. Arps in 1945. This approach was used to calculate Estimated Ultimate Recovery (EUR) for conventional oil and gas wells. Arp's presented three types of production decline rates during boundary dominated flow: Exponential, Hyperbolic and Harmonic. Moreover, Arp's derived the equations based on the value of the decline exponent constant $b$. The derivative of the loss-ratio concept was used for all three types (Arps 1945):

Loss ratio:

$$
\frac{1}{D}=-\frac{q}{d q / d t}
$$

Loss ratio derivative:

$$
n=\frac{d}{d t}=\left[-\frac{q}{\frac{d q}{d t}}\right]
$$


- The above equation 2.2.1 can be derived for the case of pseudo-steady state flow on the wells producing under constant bottomhole flowing pressure.

- Arp's derived the hyperbolic decline model, assuming constant loss ratio, $\boldsymbol{n}$ :

$$
q=q i(1+n D i t)^{\frac{-1}{n}}
$$

Where: $q=$ flow rate

$q i=$ initial flow rate

$n=$ Arps hyperbolic parameter with value between 0 and 1 for the BDF

$D i=$ initial decline rate

$\mathrm{t}=$ production time

Based on the above equations Arp's models can be:

- Exponential when $\boldsymbol{n}=\mathbf{0}$

- Harmonic when $\boldsymbol{n}=\boldsymbol{1}$

- Hyperbolic when $\boldsymbol{0} \leq \boldsymbol{n} \leq \mathbf{1}$

The value of $\boldsymbol{n}$ can indicate the decline degree of curvature and reservoir type.

Arp's models considered the value of $\boldsymbol{n}$ between 0 and 1 which can be applied for conventional reservoirs under boundary dominated flow. However, the value of $\boldsymbol{n}$ can be more than 1 for unconventional reservoirs especially for tight gas wells (Ilk. 2008). Hence, the decline curve decreases when $\boldsymbol{n}>\boldsymbol{1}$ and this can lead to invalid forecasting. Therefore, scientists have developed new equations for unconventional reservoirs with $\boldsymbol{n}$ value greater than 1 . The recently developed methods are the Power Law Exponential Decline (PLE), Stretched Exponential Decline Model (SEPD) and Duong Model. 


\subsection{Power Law Exponential Decline (PLE)}

The power law decline was developed by Ilk et al. (2008) to improve Arp's equation and better estimating the reserves for low permeability unconventional reservoirs. Moreover, PLE decline model is developed specifically for tight gas reservoirs to match early transient data and avoid over-estimation of the reserves (Seshadri, 2010).

- PLE loss-ratio model is defined by the following equation:

$$
D=D_{\infty}+n D_{i} t^{n-1}
$$

- Loss-ratio derivative:

$$
a=\frac{d}{d t}\left(\begin{array}{l}
1 \\
D
\end{array}\right)=\frac{-n(n-1) D_{i} t^{n-1}}{D^{2}}
$$

- Rate-time relation:

$$
q=q i e\left(-D_{\infty} t-\frac{D_{1}}{n} t^{n}\right)
$$

Where: $q i=$ rate intercept at $t=0$

$D_{\infty}=$ decline constant at infinite time

$D i=$ decline constant

$n=$ time exponent 


\subsection{Stretched Exponential Decline Model (SEPD)}

The stretched exponential decline model was proposed by Valko (2010) to fit transient flow regimes for the unconventional reservoirs. The model was developed with physics concepts and differential equation definition. Moreover, the new equations can handle exponent value, unlike other models.

- The below equation shows the relationship of production rate declines with time:

$$
q=q_{0} \exp \left[-\left(\frac{t}{\tau}\right)^{n}\right]
$$

Where: $q=$ time varying production rate

$q 0=$ initial production rate

$\tau=$ the characteristic time parameter

$n=$ the exponent parameter

The equation (2.4.1) looks very similar to the power law model (PLE). However, the main difference is SEPD calculation method uses two-parameters gamma functions while PLE is using a single interpretation of the parameters (Valko, 2010).

- The below expression is for cumulative production $\mathrm{Gp}$ :

$$
Q=\frac{q_{0} \tau}{n}\left\{\Gamma\left[\frac{1}{n}\right]-\Gamma\left[\frac{1}{n},\left(\frac{\tau}{n}\right)^{n}\right]\right\}
$$

- The stretched exponential decline model is more complex than other models for decline curve analysis and more difficult to apply. 


\subsection{Duong Model}

Duong has developed a new empirical model for fractured unconventional reservoirs. The preferred method of shale gas reservoirs development I s multiple-staged hydraulic fractured horizontal wells. This is due to the linear flow time of early production in tight shale reservoirs. Moreover, Duong model is considered simple and practical to use for predicting future forecast. For example, the model is capable to analyze production data and create a range of results which include the probability of reserves distribution (Duong, 2011).

- Cumulative production vs. time in log-log plot will yield a straight line regardless of fracture type (Duong, 2011).

$$
\begin{aligned}
& \frac{q}{G_{p}}=a t^{-m} \\
& q=q_{1} t(a, m)+q_{\infty}
\end{aligned}
$$

Where: $q=$ flow rate, volume/time

$$
\begin{aligned}
& a=\text { Vertical axis intercept of } \log \frac{q}{G p} \mathrm{Vs} t \\
& m=\text { slope of } \log -\log \frac{q}{G p} \mathrm{vs} t \\
& G p=\text { Cumulative gas production }
\end{aligned}
$$

Duong's model may overestimate recovery for the first ten years if the flow regime has changed during the life of the well (Vanorsdale, 2013). However, the model can predict future forecast accurately for short production history. Therefore, Joshi and Lee (2013) have modified Duong's model to accommodate the wells with longer production history. 


\section{Chapter 3: Methodology}

The main objective of this research is to apply a decline analysis method to actual production data and compare the results. Production data for five (5) Marcellus Shale wells were provided. The following procedures were followed to achieve this goal.

\subsection{Data Collection}

The data for all 5 wells were updated using the WV Geological Survey database website.

\subsection{Data Analysis}

The data were studied, and daily production rates were grouped into monthly production.

\subsection{Decline Analysis Method}

\section{Duong's Method calculation steps:}

1) Calculate $q$ and $\mathrm{Gp}$

2) Plot ( $\mathrm{q} / \mathrm{Gp}$ vs. time) in a Log-Log plot and find a \& $\mathrm{m}$ values

3) Calculate $t(a, m)$ for the given time

4) Plot q vs. $t(a, m)$ on a Cartesian graph. The straight line with the slope of qi and intercept of q $\infty$.

5) Obtain Gas rate forecasts from parts $3 \& 4$. 


\section{Chapter 4: Results and Discussion}

\subsection{Estimated Ultimate Recovery (EUR)}

- The estimated ultimate recovery using decline curve analysis were performed on all 5 wells to predict production forecast for 20 and 30 years.

- As per the chart below, well no.3 has the highest EUR production forecast while well no.2 has the lowest production among the 5 wells.

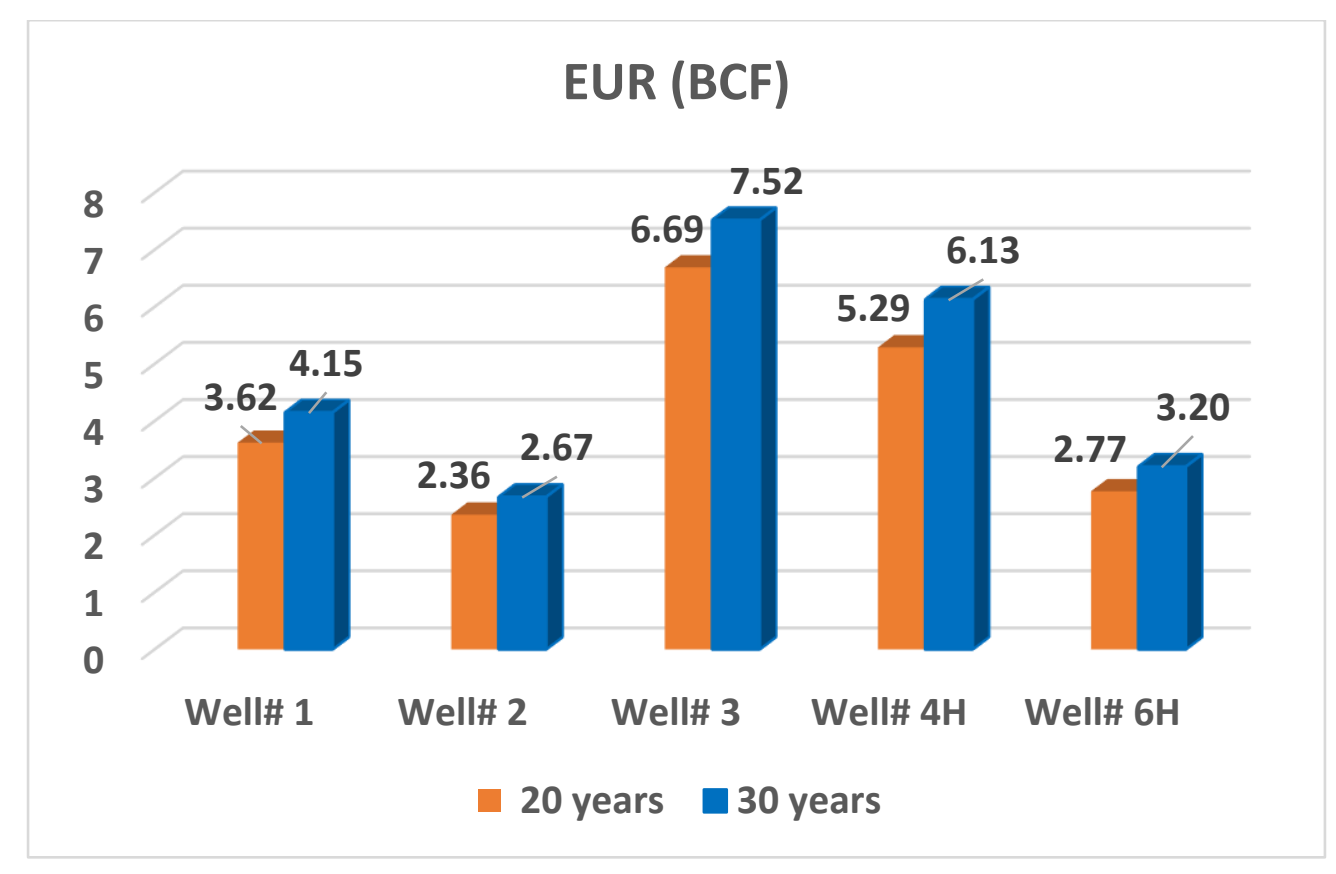

Figure 1: Estimated Ultimate Recovery (EUR) 


\section{Discussion}

Decline analysis for five (5) Marcellus Shale wells was performed with Duong's method using actual production data. Moreover, a comparison of Arp's model and Duong model was also performed for well no.1. The results were compared as illustrated below.

The analysis was performed for the wells: 1,2 and 3 using the first 48 months of production data. The later part of production was utilized for prediction and comparison as shown in the figures below. However, remaining wells, no. $4 \mathrm{H}$ and $6 \mathrm{H}$, the first 36 months was used for analysis and the remaining data was utilized for prediction and comparison. Most shale wells produce water at the beginning (frac fluid flow-back). During this early period of production, it is inclining. Therefore, the analysis was performed when the decline begins. Hence, the early production data was eliminated. In addition, for all the wells with exception of well no. 3, the decline trend is smooth. However, well no.3 shows significant fluctuations. Therefore, the data was smoothed out prior to the decline analysis to be more realistic. 


\section{Results}

- Well No.1

$\checkmark$ Field data: 111 months (Sample calculations)

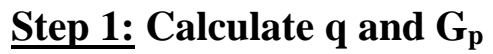

Table 1: Well No.1 Field Data

\begin{tabular}{|c|c|c|c|c|}
\hline \multicolumn{2}{|c|}{ Given Field Data } & \multicolumn{1}{c|}{} \\
\cline { 1 - 1 } \multicolumn{2}{|c|}{ Time } & $\begin{array}{c}\text { Monthly } \\
\text { Flow Rate }\end{array}$ & $\begin{array}{c}\text { Average } \\
\text { Daily } \\
\text { Flow Rate }\end{array}$ & Cumulative \\
\hline Month & Days & Mscf/Month & $\begin{array}{c}\text { q } \\
\text { (Mscf/day) }\end{array}$ & G (Mscf) \\
\hline 1 & 30 & 58056 & 1935.2 & 58056 \\
\hline 2 & 60 & 53757 & 1791.9 & 111813 \\
\hline 3 & 90 & 47962 & 1598.73333 & 159775 \\
\hline 4 & 120 & 43370 & 1445.66667 & 203145 \\
\hline 5 & 150 & 42172 & 1405.73333 & 245317 \\
\hline
\end{tabular}

Step 2: Plot ( $q / G_{p}$ vs. time) in a $\log -\log$ plot and find a \& $m$

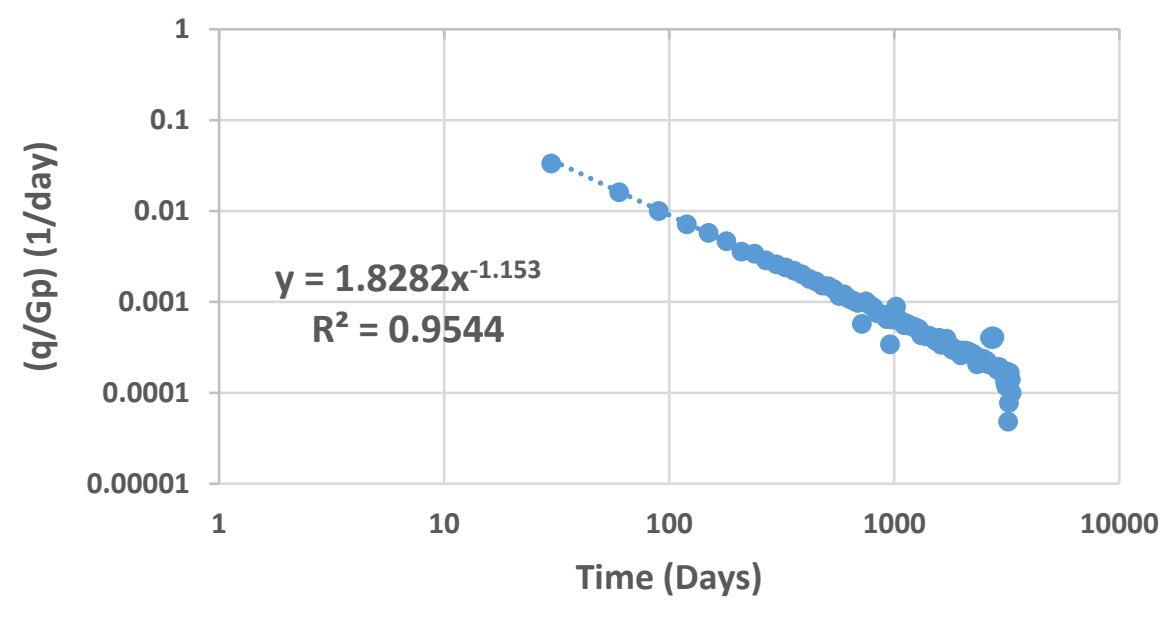

Figure 2: Well No.1 q/G $\mathbf{G}_{\mathrm{p}}$ vs. time 


\section{Step 3: Calculate t-(a,m)}

\begin{tabular}{|l|l|}
\hline$\underline{\mathbf{a}}$ & $\underline{1.828}$ \\
\hline$\underline{\underline{\mathbf{m}}}$ & $\underline{1.153}$ \\
\hline
\end{tabular}

$$
t_{(a, m)}=t^{-m_{e}}\left[\frac{a}{1-m}\left(t^{1-m}-1\right)\right]
$$

Table 2: Well No.1 t-(a,m)

\begin{tabular}{|c|c|c|c|}
\hline $\mathbf{q}$ (Mscf) & $\begin{array}{c}\mathbf{G}_{\mathbf{p}} \\
(\mathbf{M s c f})\end{array}$ & $\begin{array}{c}(\mathbf{q} / \mathbf{G p}) \\
(\mathbf{1} / \mathbf{d a y})\end{array}$ & $\underline{\mathbf{t}_{(a, m)}}$ \\
\hline 1935.2 & 58056 & 0.033333333 & 2.523615786 \\
\hline 1791.9 & 111813 & 0.016025865 & 2.318573623 \\
\hline 1598.733 & 159775 & 0.010006154 & 2.133096522 \\
\hline 1445.667 & 203145 & 0.007116428 & 1.982426625 \\
\hline 1405.733 & 245317 & 0.005730273 & 1.858580988 \\
\hline
\end{tabular}

Step 4: Plot q-vs. $\underline{\mathbf{t}}(\mathrm{a}, \mathrm{m})$ on a Cartesian graph. The straight line with the slope of qi and intercept of qo. $\quad q=q_{1} t_{(a, m)}+q_{\infty}$

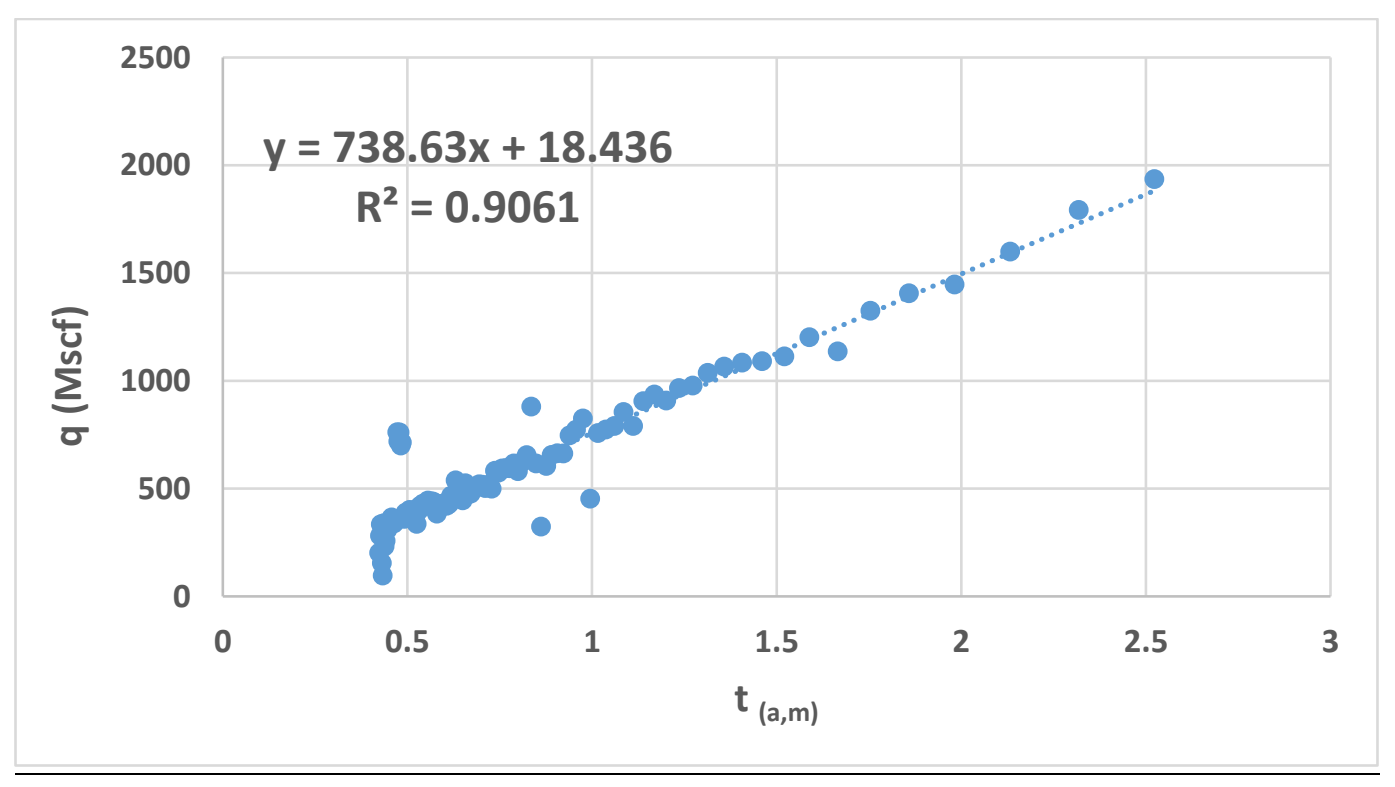

Figure 3: Well No.1 q vs. $t_{(a, m)}$ 


\begin{tabular}{|c|c|}
\hline $\mathbf{q i}$ & 738.63 \\
\hline $\mathbf{q} \infty$ & 18.44 \\
\hline
\end{tabular}

Where: $q=$ flow rate, volume/time

$$
\begin{aligned}
& a=\text { Vertical axis intercept of } \log \frac{q}{G p} \text { vs } t \\
& m=\text { slope of } \log -\log \frac{q}{G p} \text { vs } t \\
& G p=\text { Cumulative gas production }
\end{aligned}
$$

\begin{tabular}{|c|c|c|c|c|c|c|c|c|c|}
\hline \multicolumn{3}{|c|}{ Given Field Data } & & & & & & & \\
\hline \multicolumn{2}{|c|}{ Time } & \multicolumn{3}{|c|}{ Flow Rate } & \multirow[b]{2}{*}{$\begin{array}{l}\text { (q/Gp) } \\
\text { (1/day) }\end{array}$} & \multirow[b]{2}{*}{$\mathbf{t}(\mathbf{a}, \mathbf{m})$} & \multirow[b]{2}{*}{$\begin{array}{l}\text { q-Duong } \\
\text { (Mscf/d) }\end{array}$} & \multirow[b]{2}{*}{$\begin{array}{r}\text { q-Duong } \\
\text { (Mscf/m) }\end{array}$} & \multirow[b]{2}{*}{$\begin{array}{c}\text { Gp_Doung } \\
\text { (Mscf) }\end{array}$} \\
\hline Month & Days & $\begin{array}{l}\text { Monthly } \\
\text { (Mscf) }\end{array}$ & $\begin{array}{c}\text { Daily } \\
\text { (Mscf/d) }\end{array}$ & $\begin{array}{c}\text { Cum. } \\
\text { Gp } \\
\text { (Mscf) }\end{array}$ & & & & & \\
\hline 1 & 30 & 58056 & 1935.20 & 58056 & 0.033333333 & 2.5236 & 1882.46 & 56473.75 & 56473.75 \\
\hline 2 & 60 & 53757 & 1791.90 & 111813 & 0.016025865 & 2.3185 & 1731.01 & 51930.24 & 108403.99 \\
\hline 3 & 90 & 47962 & 1598.73 & 159775 & 0.010006154 & 2.1330 & 1594.01 & 47820.27 & 156224.26 \\
\hline 4 & 120 & 43370 & 1445.67 & 203145 & 0.007116428 & 1.9824 & 1482.72 & 44481.59 & 200705.86 \\
\hline 5 & 150 & 42172 & 1405.73 & 245317 & 0.005730273 & 1.8585 & 1391.24 & 41737.31 & 242443.17 \\
\hline
\end{tabular}

Step 5: Gas rate forecasts can be obtained from

Table 3: Well No.1 Gp_Doung (Mscf)

\section{Well No.1 Estimated Ultimate Recovery (EUR)}

The estimated ultimate recovery was calculated using decline curve analysis to predict production forecast for 20 and 30 years.

\begin{tabular}{|c|}
\hline EUR (BCF) for 20 years \\
\hline 3.62 \\
\hline EUR (BCF) for 30 years \\
\hline 4.15 \\
\hline
\end{tabular}




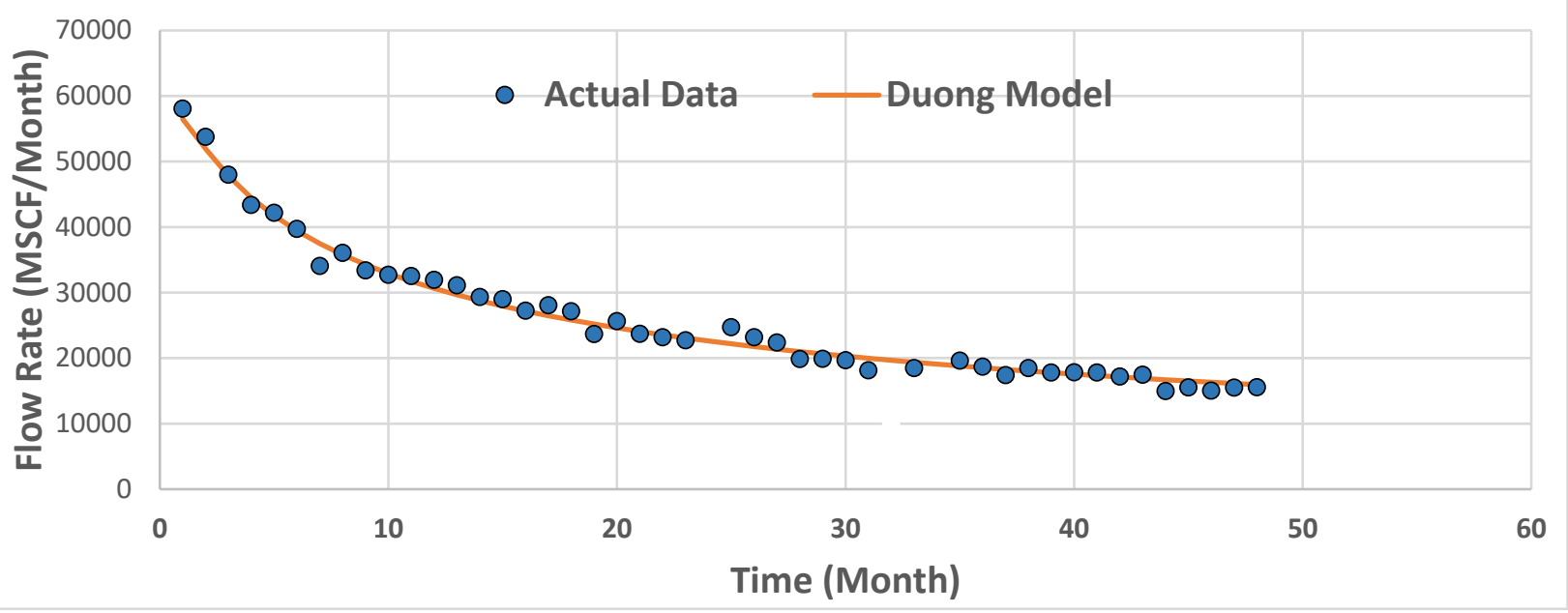

Figure 4: History Matching Well No.1 Gas Flow Rates with Duong Model

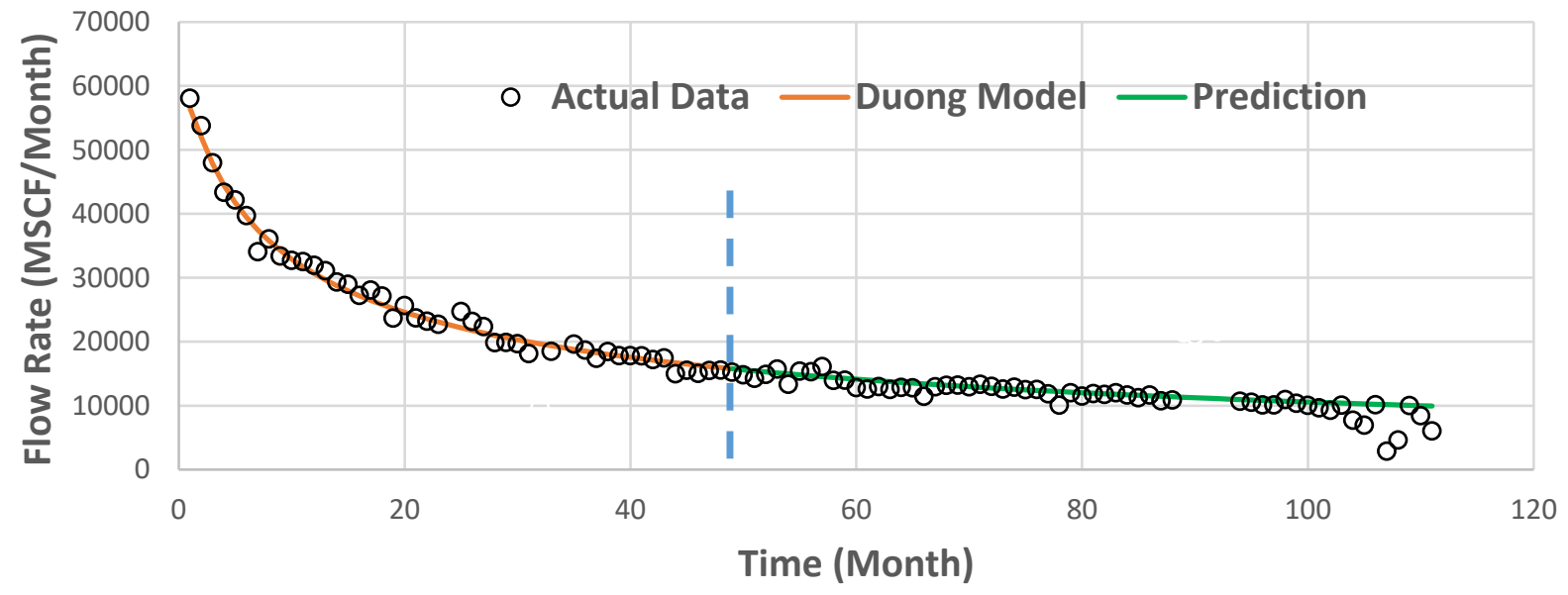

Figure 5: Comparison of the Predicted and Actual Gas Flow Rates for Well No.1 


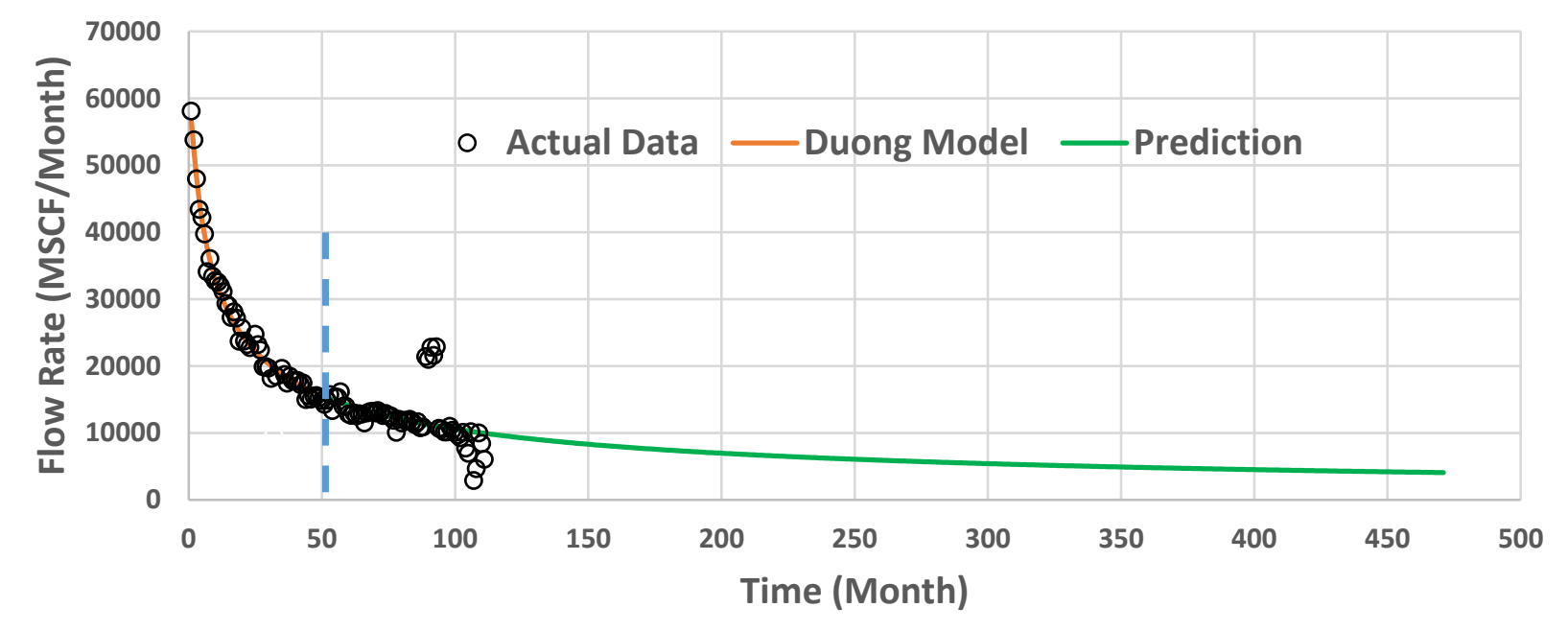

Figure 6: Predicted Gas Flow Rates for Well No.1 by Duong Model

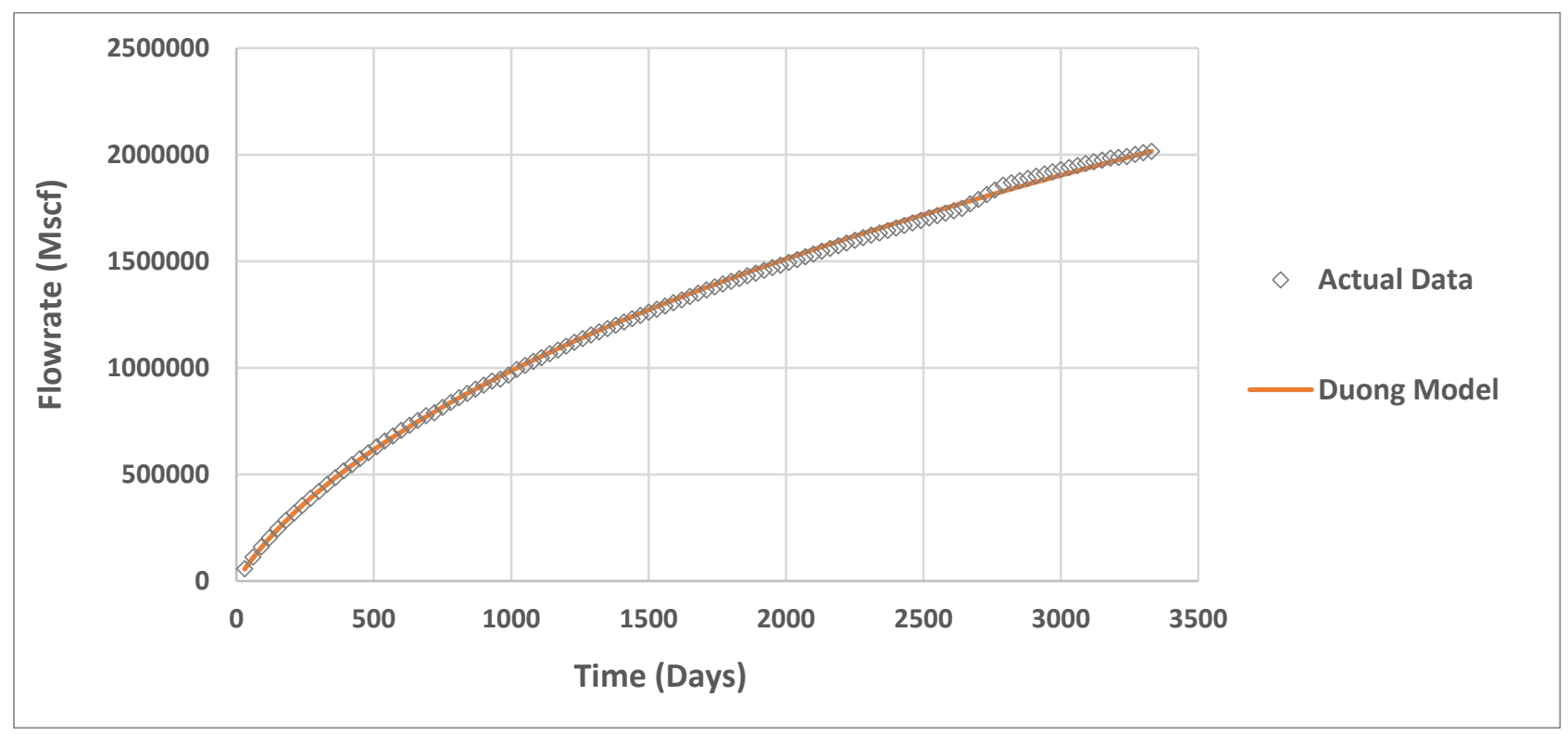

Figure 7: Comparison of Well No.1 Cumulative Production and Duong Model 
- Well No.2

$\checkmark$ Field data: 112 months (Sample calculations)

$\underline{\text { Step 1: Calculate } q \text { and } G_{p}}$

Table 4: Well No.2 Field Data

\begin{tabular}{|c|c|c|c|c|}
\hline \multicolumn{2}{|c|}{ Time } & $\begin{array}{c}\text { Monthly } \\
\text { Flow Rate }\end{array}$ & $\begin{array}{c}\text { Average } \\
\text { Daily } \\
\text { Flow Rate }\end{array}$ & Cumulative \\
\hline Month & Days & Mscf/Month & $\begin{array}{c}\text { q } \\
(\text { Mscf/day) }\end{array}$ & Gp (Mscf) \\
\hline 1 & 30 & 13662 & 455.4 & 13662 \\
\hline 2 & 60 & 44888 & 1496.26667 & 58550 \\
\hline 3 & 90 & 38747 & 1291.56667 & 97297 \\
\hline 4 & 120 & 36641 & 1221.36667 & 133938 \\
\hline 5 & 150 & 32207 & 1073.56667 & 166145 \\
\hline
\end{tabular}

Step 2: Plot ( $q / G_{p}$ vs. time) in a $\log -\log$ plot and find a \& $m$

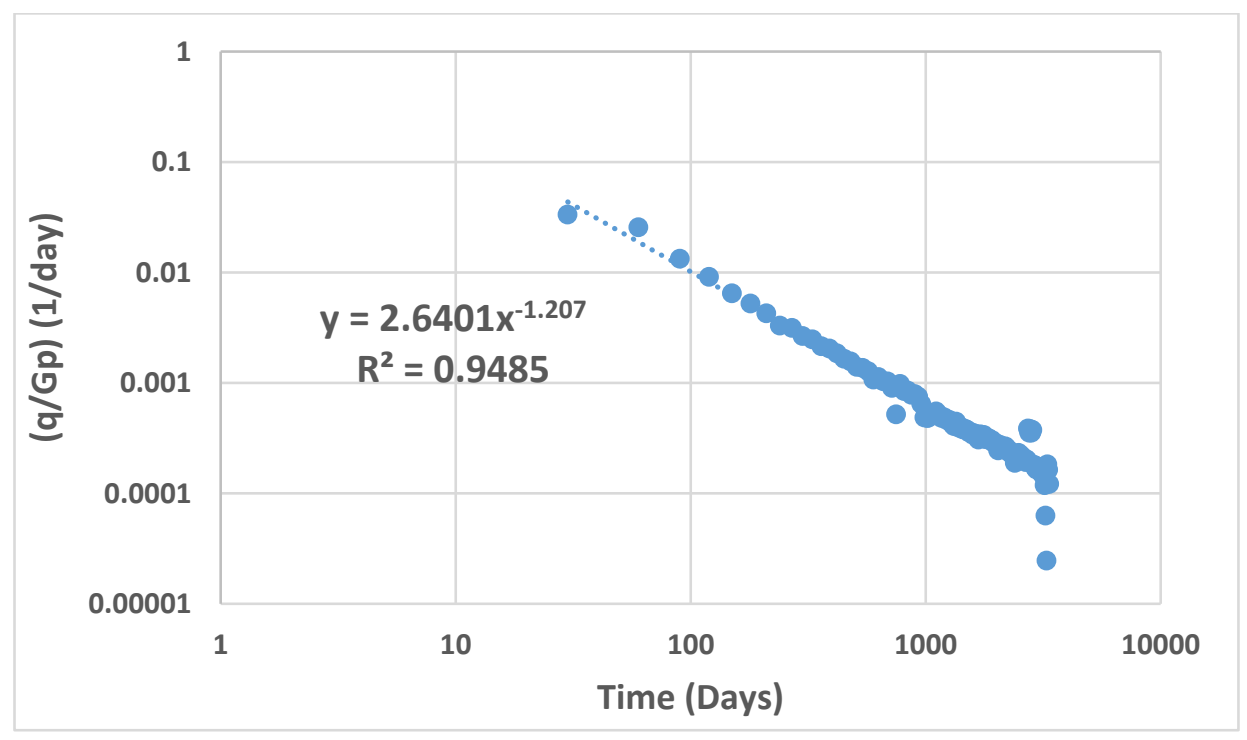

Figure 8: Well No.2 $q / G_{p}$ vs. time

\begin{tabular}{|c|c|}
\hline$\underline{\mathbf{a}}$ & $\underline{2.64}$ \\
\hline$\underline{\underline{\mathbf{m}}}$ & $\underline{1.207}$ \\
\hline
\end{tabular}


Step 3: Calculate t-(a,m)

$$
t_{(a, m)}=t^{-m_{e}} e^{\left[\frac{a}{1-m}\left(t^{1-m_{-1}}\right)\right]}
$$

Table 5: Well No.2 t-

\begin{tabular}{|c|c|c|c|}
\hline $\mathbf{q}$ (Mscf) & $\begin{array}{c}\text { Gp } \\
\text { (Mscf) }\end{array}$ & $\begin{array}{c}(\mathbf{q} / \mathbf{G p )} \\
(\mathbf{1} / \mathbf{d a y})\end{array}$ & $\mathbf{t}(\mathbf{a}, \mathbf{m})$ \\
\hline 455.4 & 13662 & 0.033333333 & 10.38851046 \\
\hline 1496.267 & 58550 & 0.025555366 & 10.45599806 \\
\hline 1291.567 & 97297 & 0.013274476 & 9.951347153 \\
\hline 1221.367 & 133938 & 0.009118896 & 9.402345861 \\
\hline 1073.567 & 166145 & 0.006461625 & 8.893545267 \\
\hline
\end{tabular}

Step 4: Plot q-vs. $\underline{\mathbf{t}}(\mathbf{a}, \mathrm{m})$ on a Cartesian graph. The straight line with the slope of qi and intercept of qळ. $\quad q=q_{1} t_{(a, m)}+q_{\infty}$

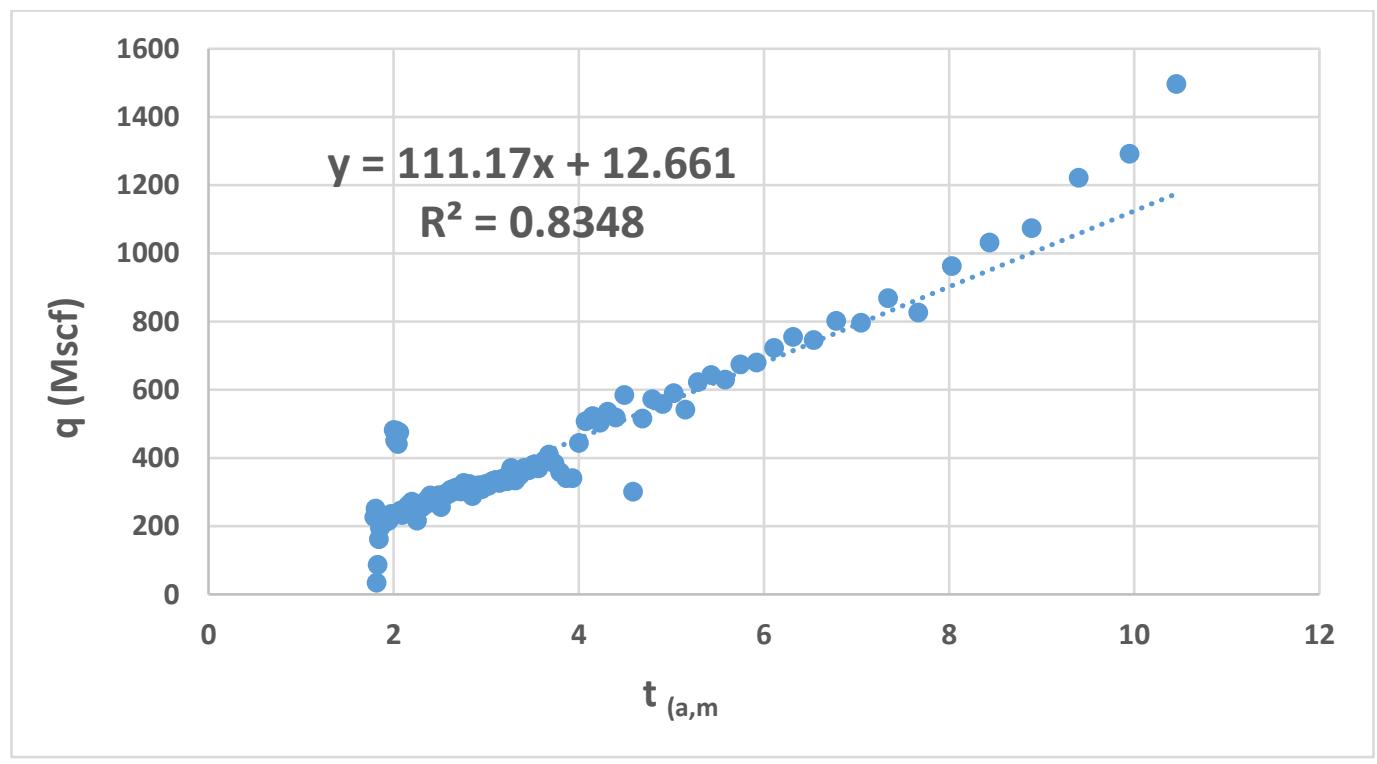

Figure 9: Well No.2 q vs. $t_{(a, m)}$ 


\begin{tabular}{|c|c|}
\hline $\mathbf{q i}$ & 111.17 \\
\hline $\mathbf{q} \infty$ & 12.66 \\
\hline
\end{tabular}

Where: $q=$ flow rate, volume/time

$$
\begin{aligned}
& a=\text { Vertical axis intercept of } \log \frac{q}{G p} \text { vs } t \\
& m=\text { slope of } \log -\log \frac{q}{G p} \text { vs } t \\
& G p=\text { Cumulative gas production }
\end{aligned}
$$

\begin{tabular}{|c|c|c|c|c|c|c|c|c|c|}
\hline \multicolumn{3}{|c|}{ Given Field Data } & & & & & & & \\
\hline \multicolumn{2}{|c|}{ Time } & \multicolumn{3}{|c|}{ Flow Rate } & \multirow[b]{2}{*}{$\begin{array}{l}(q / \mathbf{G p}) \\
(1 / \text { day })\end{array}$} & \multirow[b]{2}{*}{$\mathbf{t}(\mathbf{a}, \mathbf{m})$} & \multirow[b]{2}{*}{$\begin{array}{l}\text { q-Duong } \\
\text { (Mscf/d) }\end{array}$} & \multirow[b]{2}{*}{$\begin{array}{l}\text { q-Duong } \\
(\text { Mscf/m) }\end{array}$} & \multirow[b]{2}{*}{$\begin{array}{c}\text { Gp_Doung } \\
\text { (Mscf) }\end{array}$} \\
\hline Month & Days & $\begin{array}{c}\text { Monthly } \\
\text { (Mscf) }\end{array}$ & $\begin{array}{c}\text { Daily } \\
\text { (Mscf/d) }\end{array}$ & $\begin{array}{c}\text { Cum. } \\
\text { Gp } \\
\text { (Mscf) }\end{array}$ & & & & & \\
\hline 1 & 30 & 13662 & 455.40 & 13662 & 0.033333333 & 10.38851046 & 1167.55 & 35026.52 & 35026.52 \\
\hline 2 & 60 & 44888 & 1496.27 & 58550 & 0.025555366 & 10.45599806 & 1175.05 & 35251.60 & 70278.12 \\
\hline 3 & 90 & 38747 & 1291.57 & 97297 & 0.013274476 & 9.951347153 & 1118.95 & 33568.54 & 103846.66 \\
\hline 4 & 120 & 36641 & 1221.37 & 133938 & 0.009118896 & 9.402345861 & 1057.92 & 31737.56 & 135584.22 \\
\hline 5 & 150 & 32207 & 1073.57 & 166145 & 0.006461625 & 8.893545267 & 1001.36 & 30040.66 & 165624.88 \\
\hline
\end{tabular}

Step 5: Gas rate forecasts can be obtained from

Table 6: Well No.2 G

\section{Well No.2 Estimated Ultimate Recovery (EUR)}

The estimated ultimate recovery was calculated using decline curve analysis to predict production forecast for 20 and 30 years.

\begin{tabular}{|c|}
\hline EUR (BCF) for 20 years \\
\hline 2.36 \\
\hline EUR (BCF) for 30 years \\
\hline 2.67 \\
\hline
\end{tabular}




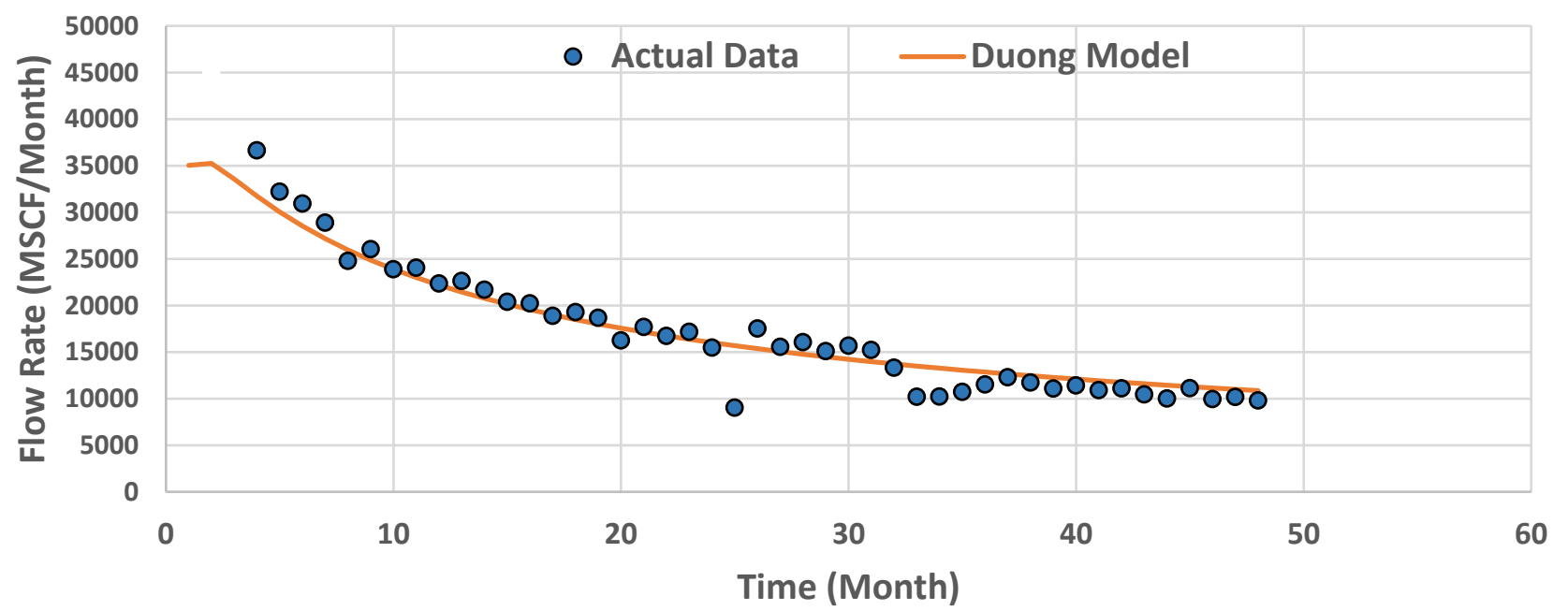

Figure 10: History Matching Well No.2 Gas Flow Rates with Duong Model

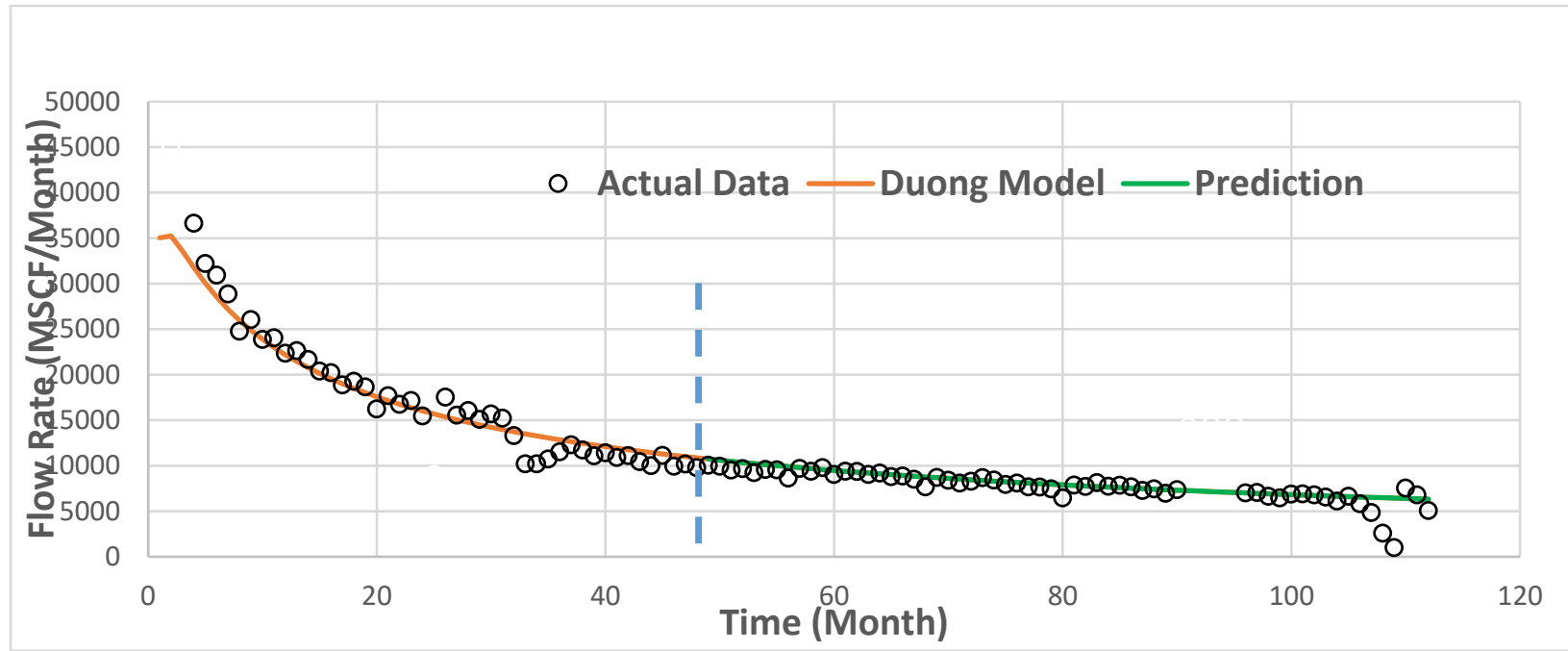

Figure 11: Comparison of the Predicted and Actual Gas Flow Rates for Well No.2 


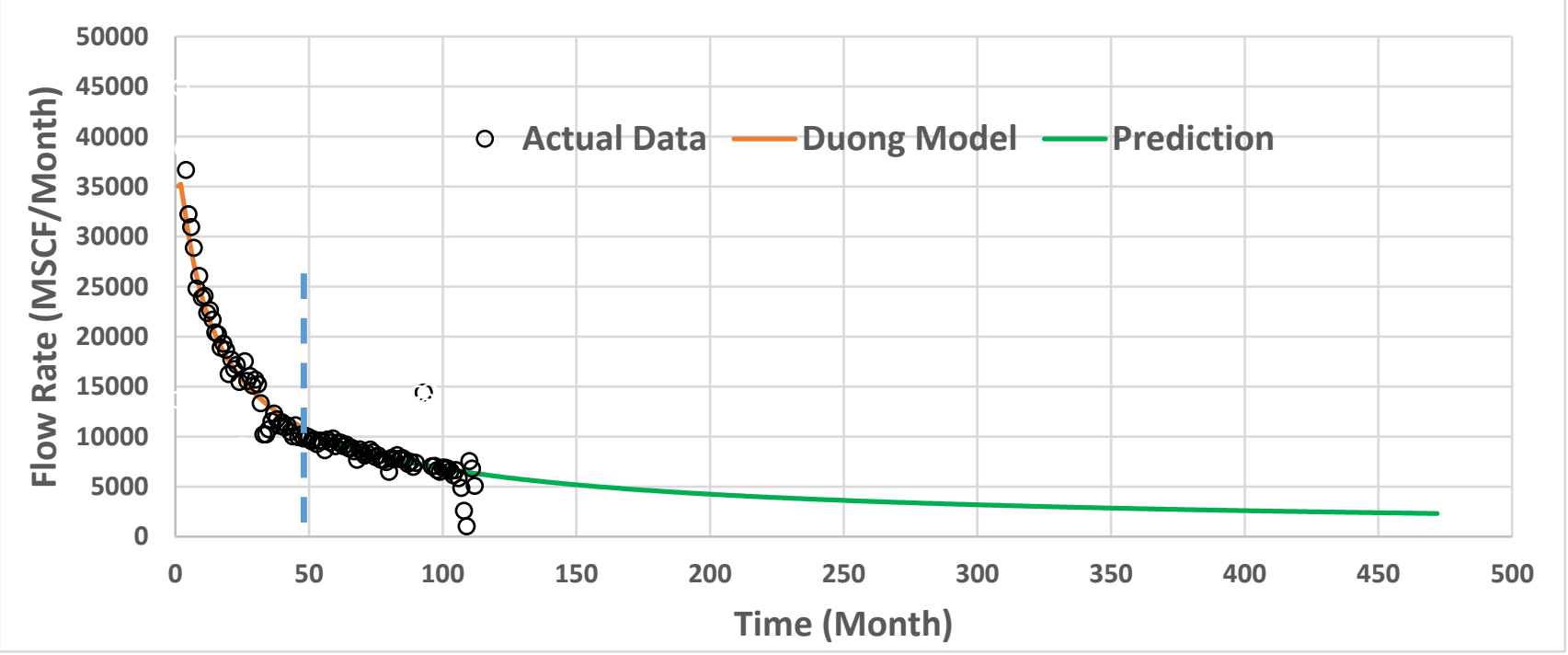

Figure 12: Predicted Gas Flow Rates for Well No.2 by Duong Model

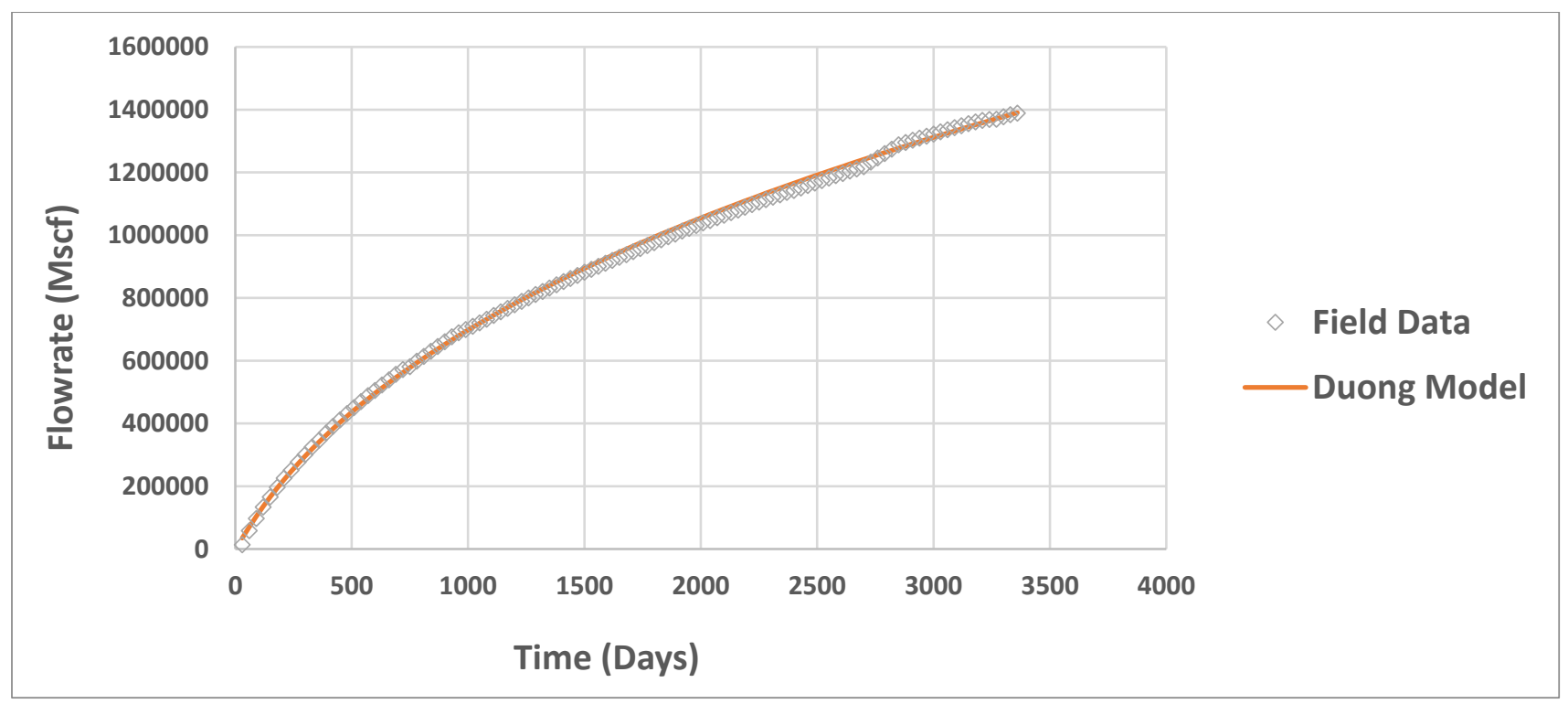

Figure 13: Comparison of Well No.2 Cumulative Production and Duong Model 
- Well No.3

$\checkmark$ Field data: 124 months (Sample calculations)

\section{Step 1: Calculate $q$ and $\mathbf{G}_{\mathbf{p}}$}

Table 7: Well No.3 Field Data

\begin{tabular}{|c|c|c|c|c|}
\hline \multicolumn{2}{|c|}{ Given Field Data } & \multicolumn{2}{c}{} \\
\cline { 1 - 3 } \multicolumn{2}{|c|}{ Time } & Monthly & $\begin{array}{c}\text { Average } \\
\text { Daily }\end{array}$ & \multirow{2}{*}{ Cumulative } \\
\hline Month & Days & Flow Rate & Flow Rate & \\
\hline 1 & 30 & 24,884 & 829.466667 & 24,884 \\
\hline 2 & 60 & 139,490 & 4649.66667 & 164374 \\
\hline 3 & 90 & 156342 & 5211.4 & 320716 \\
\hline 4 & 120 & 143525 & 4784.16667 & 464241 \\
\hline 5 & 150 & 123918 & 4130.6 & 588159 \\
\hline
\end{tabular}

Step 2: Plot ( $q / G_{p}$ vs. time) in a $\log -\log$ plot and find a \& $m$

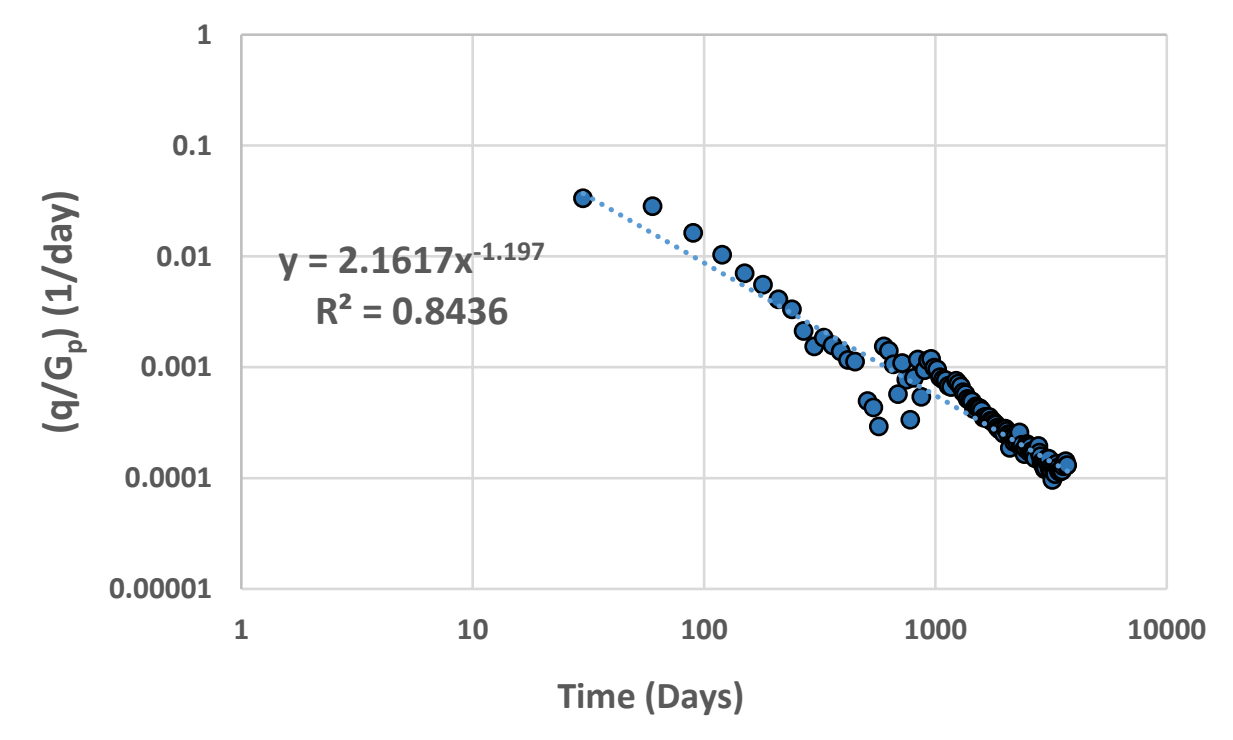

Figure 14: Well No.3 q/ $G_{p}$ vs. time

\begin{tabular}{|c|c|}
\hline$\underline{\underline{\mathbf{a}}}$ & $\underline{\underline{2.1617}}$ \\
\hline$\underline{\underline{\mathbf{m}}}$ & $\underline{\underline{1.197}}$ \\
\hline
\end{tabular}


Step 3: Calculate t-(a,m)

$$
t_{(a, m)}=t^{-m_{e}}\left[\frac{a}{1-m}\left(t^{1-m}-1\right)\right]
$$

Table 8: Well No.3 t- $\underline{\underline{(a, m)}}$

\begin{tabular}{|c|c|c|c|}
\hline $\mathbf{q}$ (Mscf) & $\mathbf{G}_{\mathbf{p}}$ (Mscf) & $\begin{array}{c}(\mathbf{q} / \mathbf{G p}) \\
(\mathbf{1} / \mathbf{d a y})\end{array}$ & $\underline{\mathbf{t}}_{(\mathbf{a}, \mathbf{m})}$ \\
\hline 829.4667 & 24,884 & 0.033333333 & 3.622038871 \\
\hline 4649.667 & 164374 & 0.028287118 & 3.234910061 \\
\hline 5211.4 & 320716 & 0.016249267 & 2.899940807 \\
\hline 4784.167 & 464241 & 0.010305351 & 2.636605944 \\
\hline 4130.6 & 588159 & 0.007022931 & 2.425791453 \\
\hline
\end{tabular}

Step 4: Plot q-vs. $\underline{t}$ (a,m) on a Cartesian graph. The straight line with the slope of qi and intercept of qळ. $\quad q=q_{1} t_{(a, m)}+q_{\infty}$

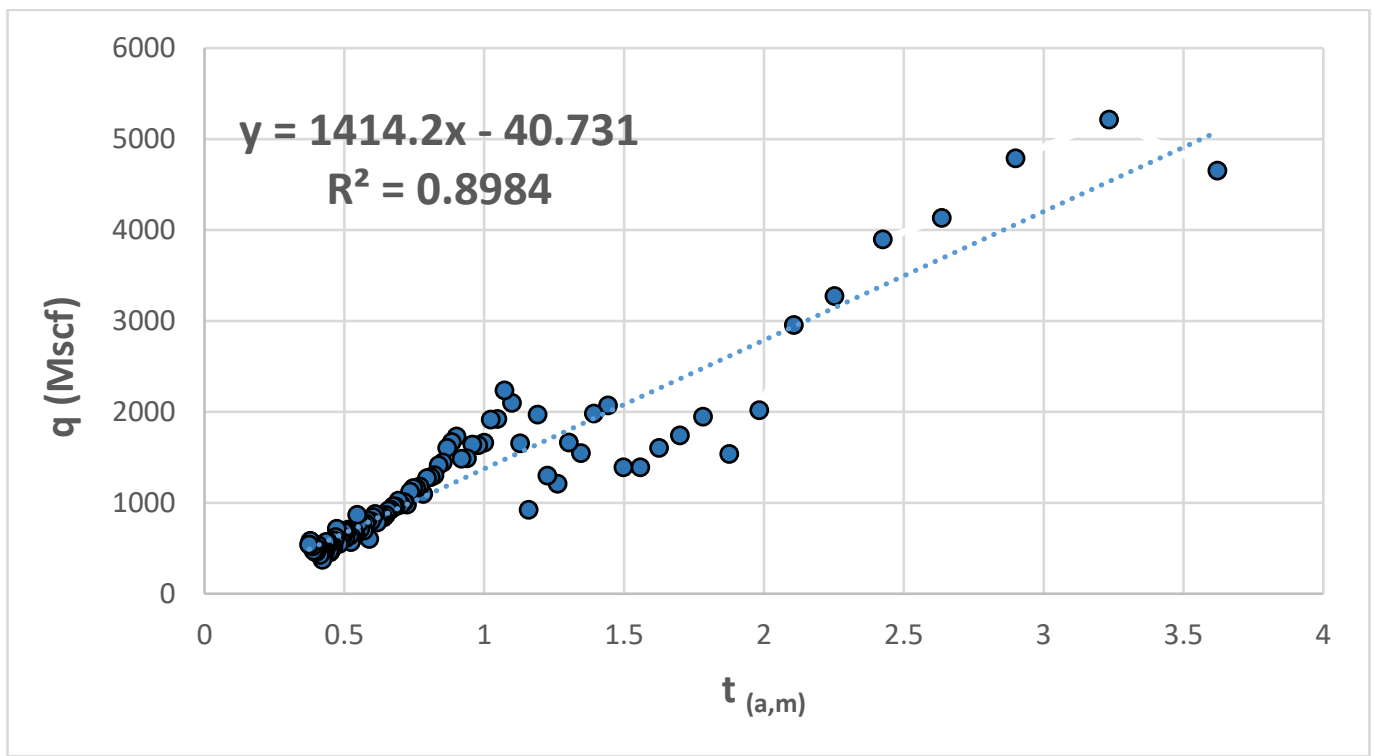

Figure 15: Well No.3 q vs. $t_{(a, m)}$

\begin{tabular}{|c|c|}
\hline $\mathbf{q i}$ & 1414 \\
\hline $\mathbf{q} \infty$ & 40.73 \\
\hline
\end{tabular}


Where: $q=$ flow rate, volume/time

$$
\begin{aligned}
& a=\text { Vertical axis intercept of } \log \frac{q}{G p} \text { vs } t \\
& m=\text { slope of } \log -\log \frac{q}{G p} \text { vs } t \\
& G p=\text { Cumulative gas production }
\end{aligned}
$$

\begin{tabular}{|c|c|c|c|c|c|c|c|c|c|}
\hline \multicolumn{3}{|c|}{ Given Field Data } & & & & & & & \\
\hline \multicolumn{2}{|c|}{ Time } & \multicolumn{3}{|c|}{ Flow Rate } & \multirow[b]{2}{*}{$\begin{array}{l}\text { (q/Gp) } \\
\text { (1/day) }\end{array}$} & \multirow[b]{2}{*}{$\mathbf{t}(\mathbf{a}, \mathbf{m})$} & \multirow[b]{2}{*}{$\begin{array}{c}\text { q- } \\
\text { Duong } \\
\text { (Mscf/d) }\end{array}$} & \multirow[b]{2}{*}{$\begin{array}{l}\text { q-Duong } \\
\text { (Mscf/m) }\end{array}$} & \multirow[b]{2}{*}{$\begin{array}{c}\text { Gp_Doung } \\
\text { (Mscf) }\end{array}$} \\
\hline Month & Days & $\begin{array}{l}\text { Monthly } \\
\text { (Mscf) }\end{array}$ & $\begin{array}{c}\text { Daily } \\
\text { (Mscf/d) }\end{array}$ & $\begin{array}{c}\text { Cum. } \\
\text { Gp } \\
\text { (Mscf) }\end{array}$ & & & & & \\
\hline 1 & 30 & 139,490 & 4649.67 & 139490 & 0.033333333 & 3.622038871 & 5163.02 & 154890.52 & 154890.52 \\
\hline 2 & 60 & 156342 & 5211.40 & 295832 & 0.017616079 & 3.234910061 & 4615.54 & 138466.19 & 293356.72 \\
\hline 3 & 90 & 143525 & 4784.17 & 439357 & 0.010889019 & 2.899940807 & 4141.83 & 124254.79 & 417611.50 \\
\hline 4 & 120 & 123918 & 4130.60 & 563275 & 0.007333185 & 2.636605944 & 3769.42 & 113082.54 & 530694.05 \\
\hline 5 & 150 & 116830 & 3894.33 & 680105 & 0.005726077 & 2.425791453 & 3471.28 & 104138.53 & 634832.58 \\
\hline
\end{tabular}

Step 5: Gas rate forecasts can be obtained from

Table 9: Well No.3 Gp_Doung (Mscf)

\section{Well No.3 Estimated Ultimate Recovery (EUR)}

The estimated ultimate recovery was calculated using decline curve analysis to predict production forecast for 20 and 30 years.

\begin{tabular}{|c|}
\hline EUR (BCF) for 20 years \\
\hline 6.69 \\
\hline EUR (BCF) for 30 years \\
\hline 7.52 \\
\hline
\end{tabular}




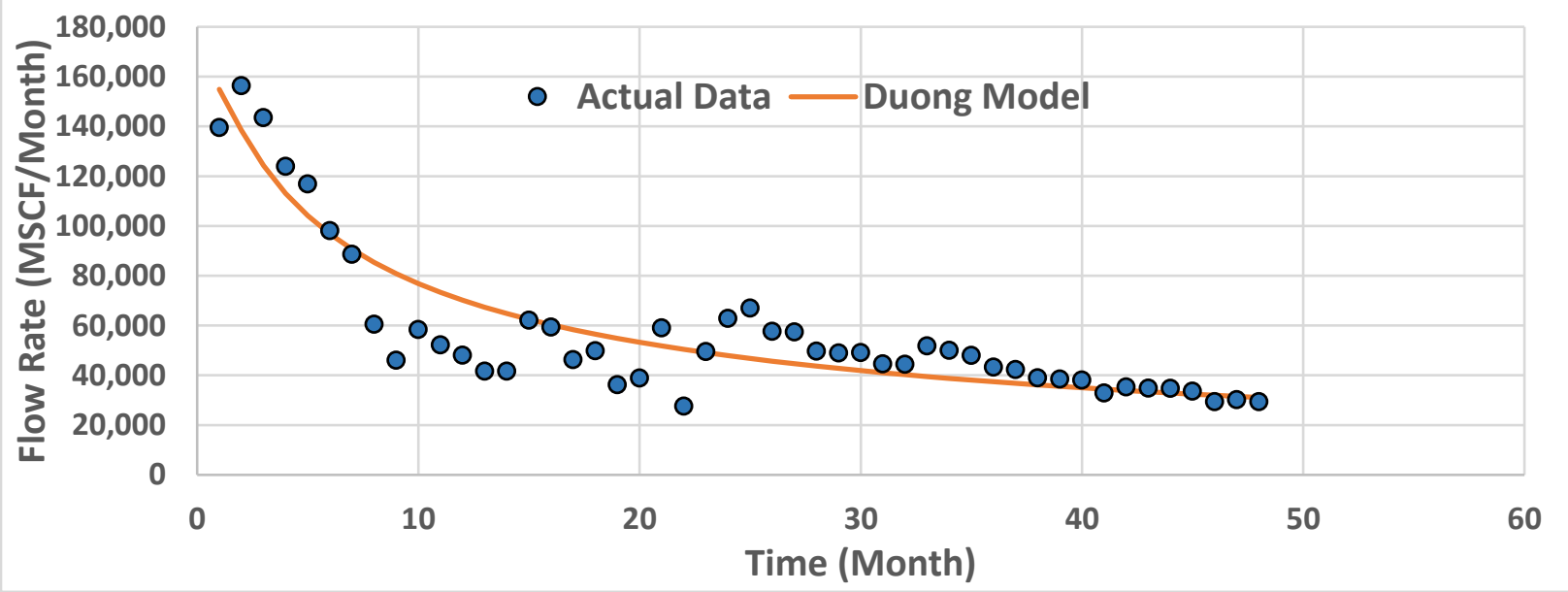

Figure 16: History Matching Well No.3 Gas Flow Rates with Duong Model

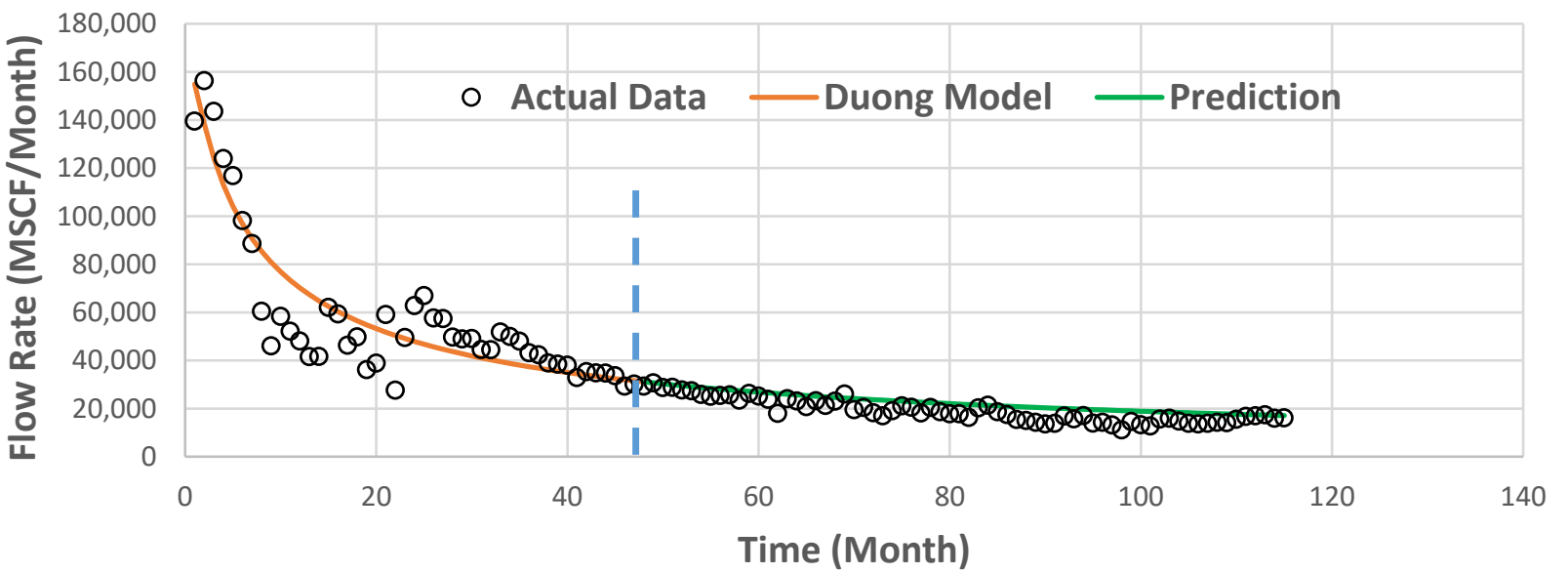

Figure 17: Comparison of the Predicted and Actual Gas Flow Rates for Well No.3 


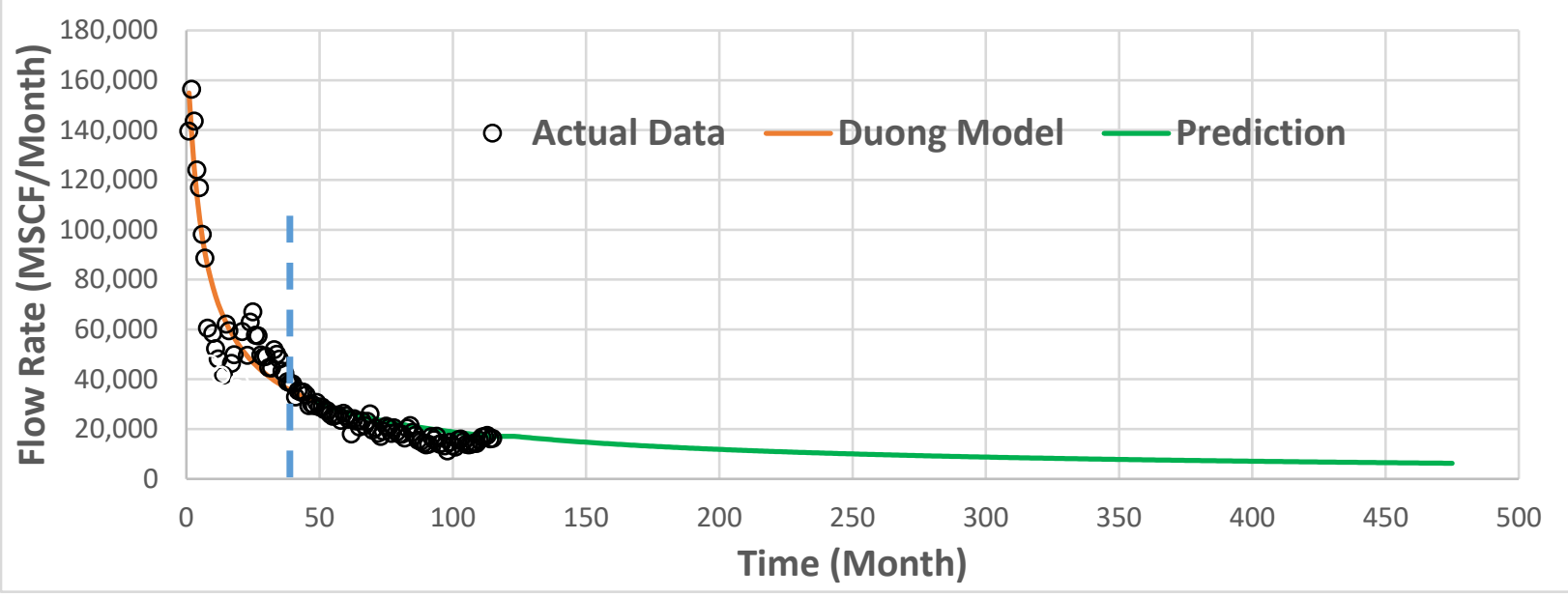

Figure 18: Predicted Gas Flow Rates for Well No.3 by Duong Model

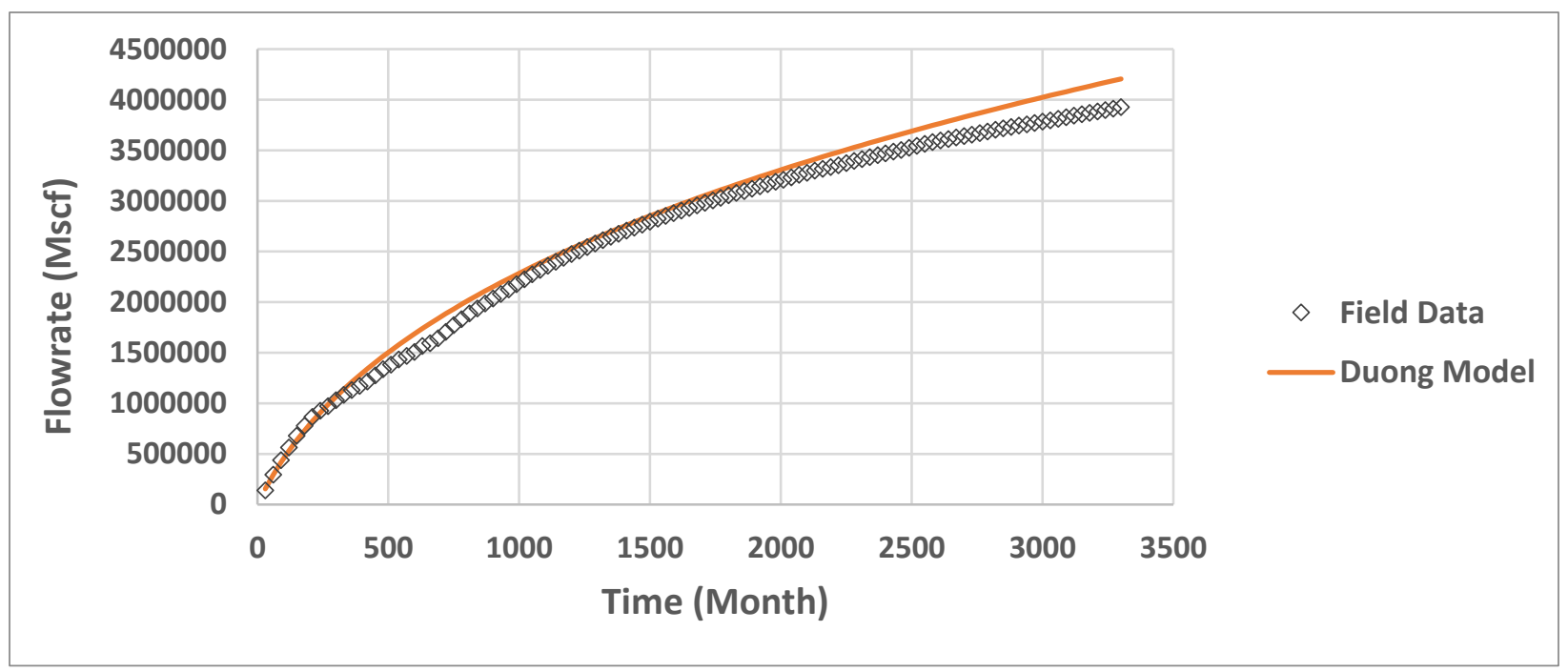

Figure 19: Well No.3 Cumulative Production vs. Duong Model 
- Well No.4H

$\checkmark$ Field data: 69 months (Sample calculations)

$\underline{\text { Step 1: Calculate } q \text { and } G_{p}}$

Table 10: Well No.4H Field Data

\begin{tabular}{|c|c|c|c|c|}
\hline \multicolumn{2}{|c|}{ Given Field Data } & \multicolumn{1}{c|}{} \\
\hline \multicolumn{2}{|c|}{ Time } & $\begin{array}{c}\text { Monthly } \\
\text { Flow Rate }\end{array}$ & $\begin{array}{c}\text { Average } \\
\text { Daily } \\
\text { Flow Rate }\end{array}$ & Cumulative \\
\hline Month & Days & Mscf/Month & $\begin{array}{c}\text { q } \\
\text { (Mscf/day) }\end{array}$ & Gp (Mscf) \\
\hline 1 & 30 & 37,032 & 1234.4 & 37032 \\
\hline 2 & 60 & 82,849 & 2761.63333 & 119881 \\
\hline 3 & 90 & 77,704 & 2590.13333 & 197585 \\
\hline 4 & 120 & 76,042 & 2534.73333 & 273627 \\
\hline 5 & 150 & 71,697 & 2389.9 & 345324 \\
\hline
\end{tabular}

Step 2: Plot ( $q / G_{p}$ vs. time) in a $\log -\log$ plot and find a \& $m$

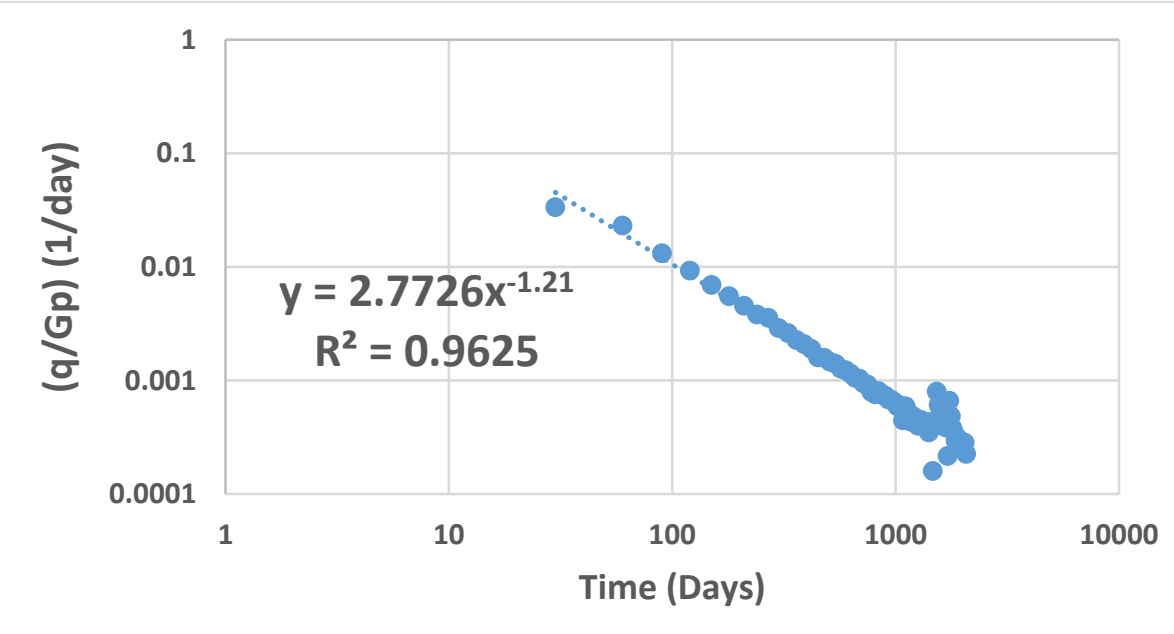

Figure 20: Well No.4H q/G $/ G_{p}$ vs. time 
Step 3: Calculate t-(a,m)

\begin{tabular}{|c|c|}
\hline$\underline{\underline{\mathbf{a}}}$ & $\underline{\underline{2.773}}$ \\
\hline$\underline{\underline{\mathbf{m}}}$ & $\underline{\underline{1.21}}$ \\
\hline
\end{tabular}

$$
t_{(a, m)}=t^{-m_{e}}\left[\frac{a}{1-m}\left(t^{1-m_{-1}}\right)\right]
$$

Table 11: Well No.4H t- $(\mathbf{a}, \mathrm{m})$

\begin{tabular}{|c|c|c|c|}
\hline $\mathbf{q}$ (Mscf) & $\begin{array}{c}\text { Gp } \\
\text { (Mscf) }\end{array}$ & $\begin{array}{c}(\mathbf{q} / \mathbf{G p}) \\
(\mathbf{1} / \mathbf{d a y})\end{array}$ & $\mathbf{t}(\mathbf{a}, \mathbf{m})$ \\
\hline 1234.4 & 37032 & 0.033333333 & 13.80195873 \\
\hline 2761.633 & 119881 & 0.023036456 & 14.32207202 \\
\hline 2590.133 & 197585 & 0.013108957 & 13.83727267 \\
\hline 2534.733 & 273627 & 0.009263462 & 13.19936211 \\
\hline 2389.9 & 345324 & 0.006920747 & 12.57055624 \\
\hline
\end{tabular}

Step 4: Plot q-vs. $\underline{\mathbf{t}}(\mathrm{a}, \mathrm{m})$ on a Cartesian graph. The straight line with the slope of qi and intercept of qळ. $\quad q=q_{1} t_{(a, m)}+q_{\infty}$

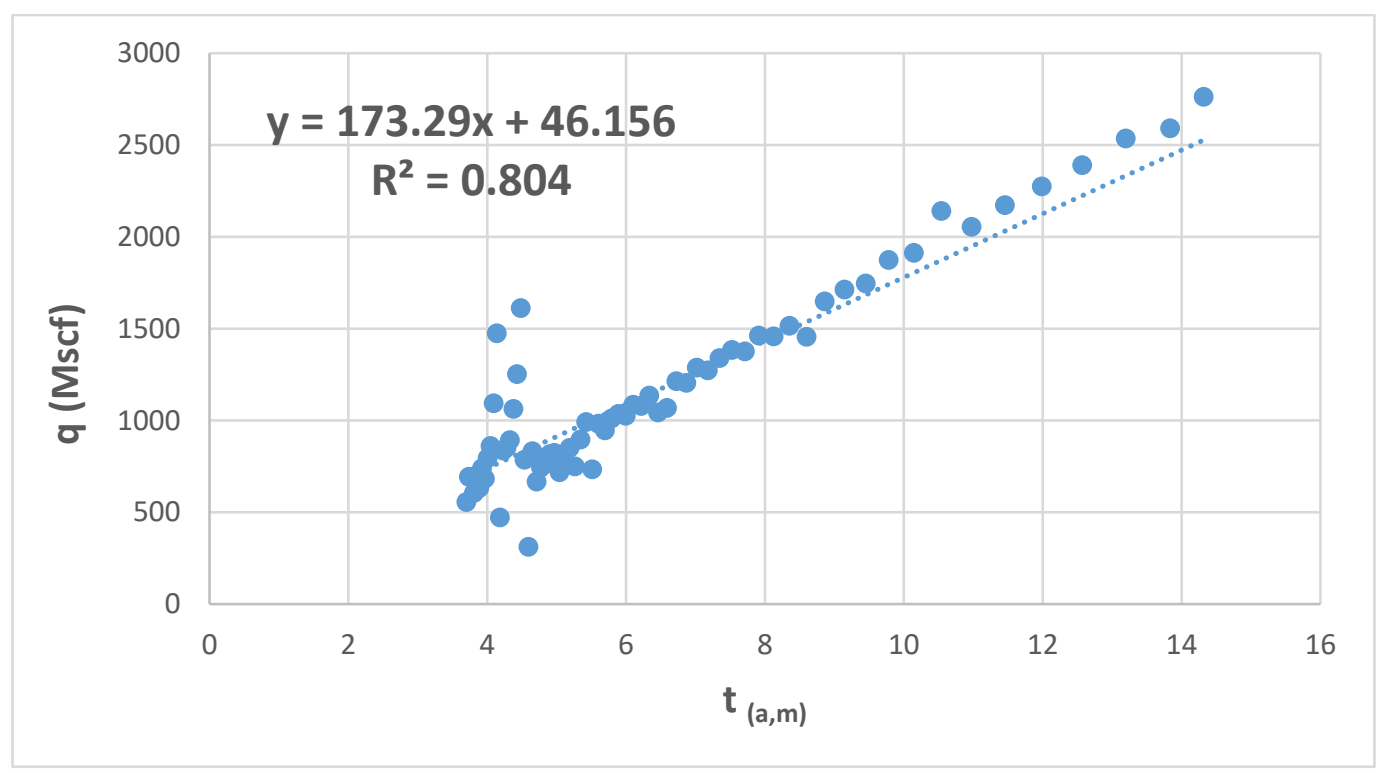

Figure 21: Well No.4H q vs. $t_{(a, m)}$ 


\begin{tabular}{|c|c|}
\hline $\mathbf{q i}$ & 173.29 \\
\hline $\mathbf{q} \infty$ & 46.156 \\
\hline
\end{tabular}

Where: $q=$ flow rate, volume/time

$$
\begin{aligned}
& a=\text { Vertical axis intercept of } \log \frac{q}{G p} \text { vs } t \\
& m=\text { slope of } \log -\log \frac{q}{G p} \text { vs } t \\
& G p=\text { Cumulative gas production }
\end{aligned}
$$

\begin{tabular}{|c|c|c|c|c|c|c|c|c|c|}
\hline \multicolumn{3}{|c|}{ Given Field Data } & & & & & & & \\
\hline \multicolumn{2}{|c|}{ Time } & \multicolumn{3}{|c|}{ Flow Rate } & \multirow[b]{2}{*}{$\begin{array}{l}\text { (q/Gp) } \\
\text { (1/day) }\end{array}$} & \multirow[b]{2}{*}{$\mathbf{t}(\mathbf{a}, \mathbf{m})$} & \multirow[b]{2}{*}{$\begin{array}{c}\text { q- } \\
\text { Duong } \\
\text { (Mscf/d) }\end{array}$} & \multirow[b]{2}{*}{$\begin{array}{l}\text { q-Duong } \\
\text { (Mscf/m) }\end{array}$} & \multirow[b]{2}{*}{$\begin{array}{c}\text { Gp_Doung } \\
\text { (Mscf) }\end{array}$} \\
\hline Month & Days & $\begin{array}{c}\text { Monthly } \\
\text { (Mscf) }\end{array}$ & $\begin{array}{c}\text { Daily } \\
\text { (Mscf/d) }\end{array}$ & $\begin{array}{c}\text { Cum. } \\
\text { Gp } \\
\text { (Mscf) }\end{array}$ & & & & & \\
\hline 1 & 30 & 37,032 & 1234.40 & 37032 & 0.033 & 13.80 & 2437.90 & 73136.92 & 73136.92 \\
\hline 2 & 60 & 82,849 & 2761.63 & 119881 & 0.023 & 14.32 & 2528.03 & 75840.84 & 148977.76 \\
\hline 3 & 90 & 77,704 & 2590.13 & 197585 & 0.013 & 13.83 & 2444.02 & 73320.51 & 222298.27 \\
\hline 4 & 120 & 76,042 & 2534.73 & 273627 & 0.009 & 13.19 & 2333.47 & 70004.20 & 292302.47 \\
\hline 5 & 150 & 71,697 & 2389.90 & 345324 & 0.006 & 12.57 & 2224.51 & 66735.23 & 359037.70 \\
\hline
\end{tabular}

Step 5: Gas rate forecasts can be obtained from

Table 12: Well No.4H Gp_Doung (Mscf)

\section{Well No.4 Estimated Ultimate Recovery (EUR)}

The estimated ultimate recovery was calculated using decline curve analysis to predict production forecast for 20 and 30 years.

\begin{tabular}{|c|}
\hline EUR (BCF) for 20 years \\
\hline 5.29 \\
\hline EUR (BCF) for 30 years \\
\hline 6.13 \\
\hline
\end{tabular}




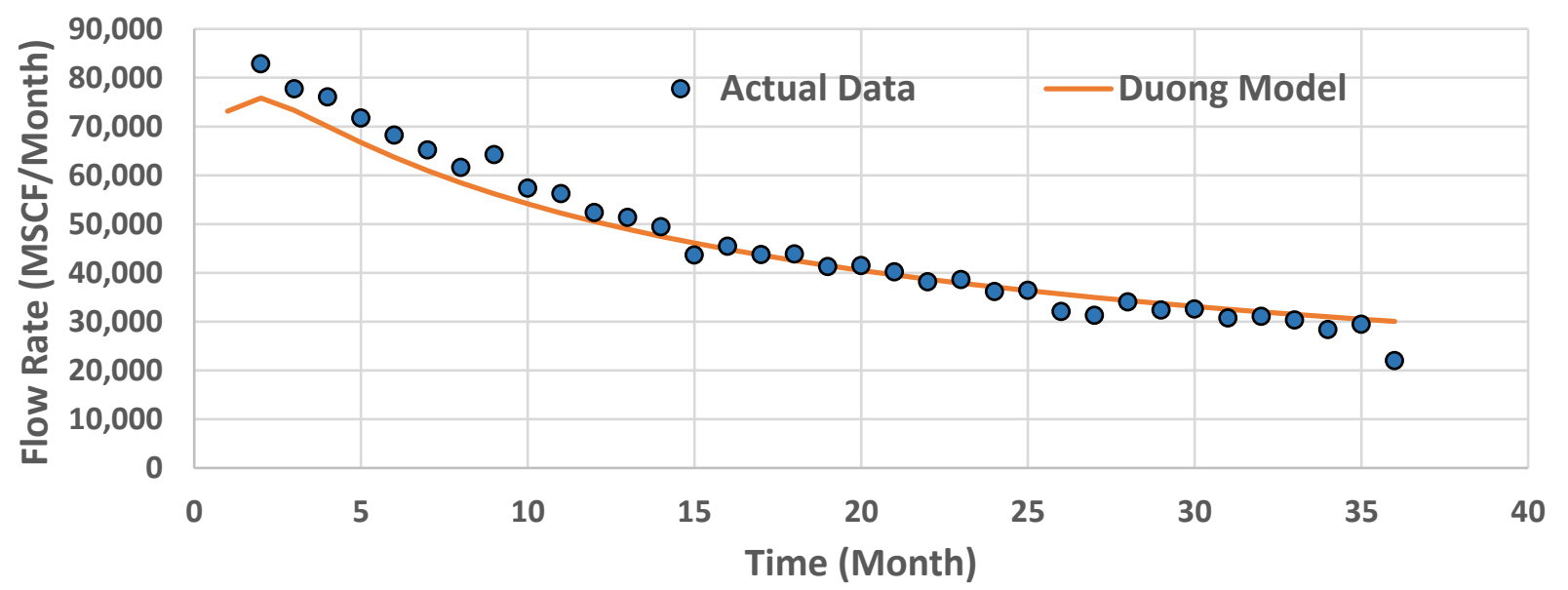

Figure 22: History Matching Well No.4H Gas Flow Rates with Duong Model

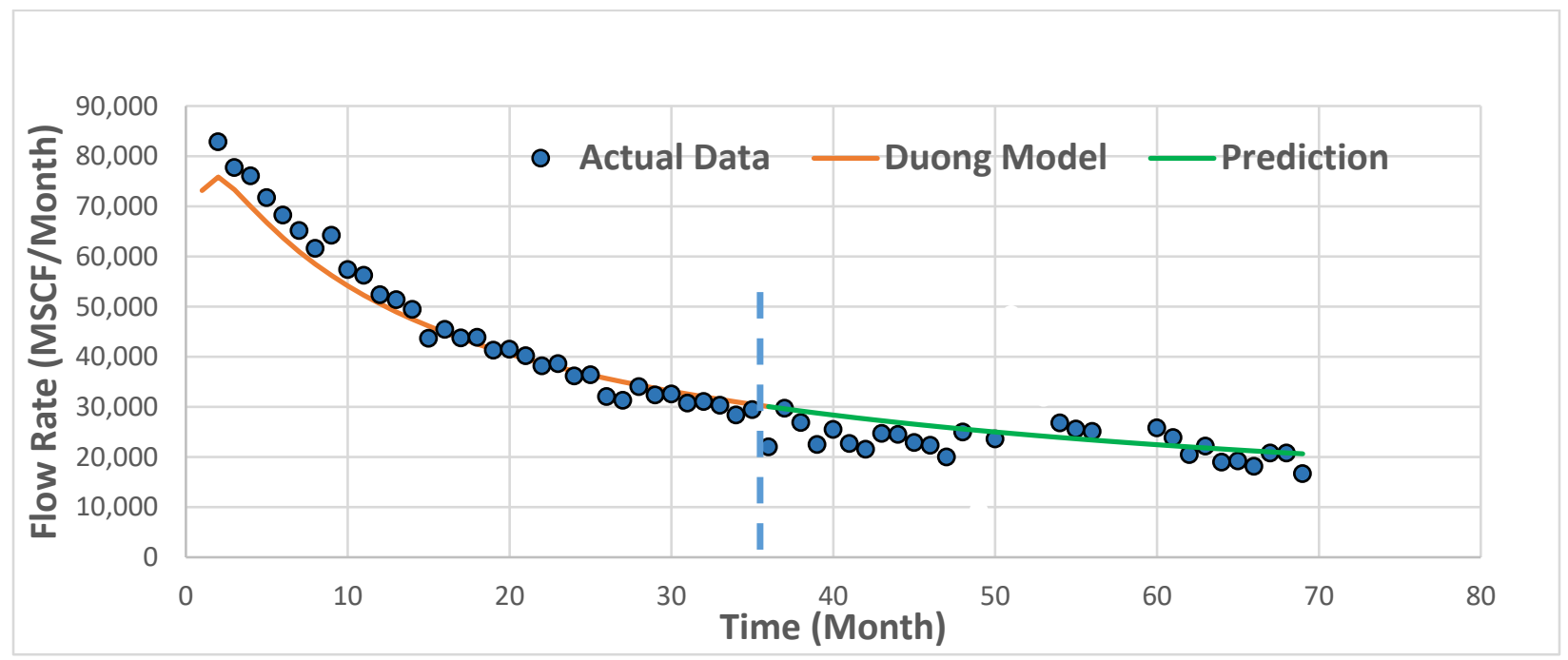

Figure 23: Comparison of the Predicted and Actual Gas Flow Rates for Well No.4H 


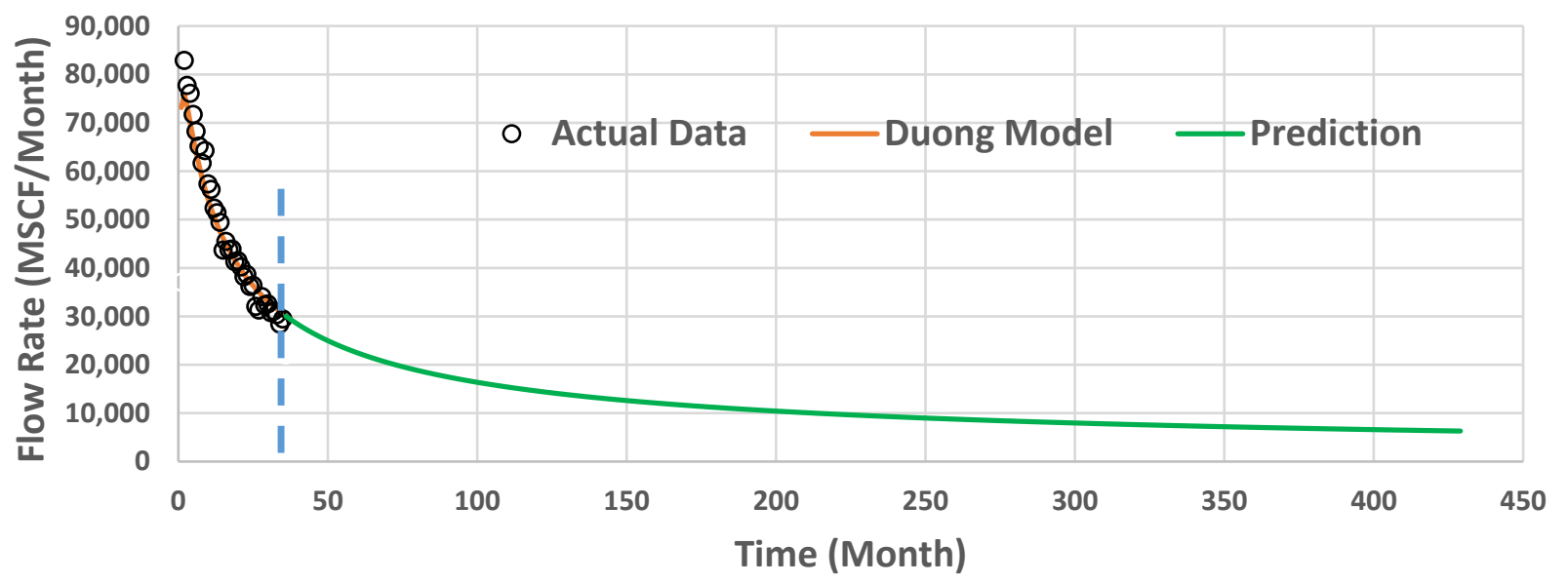

Figure 24: Predicted Gas Flow Rates for Well No.4H by Duong Model

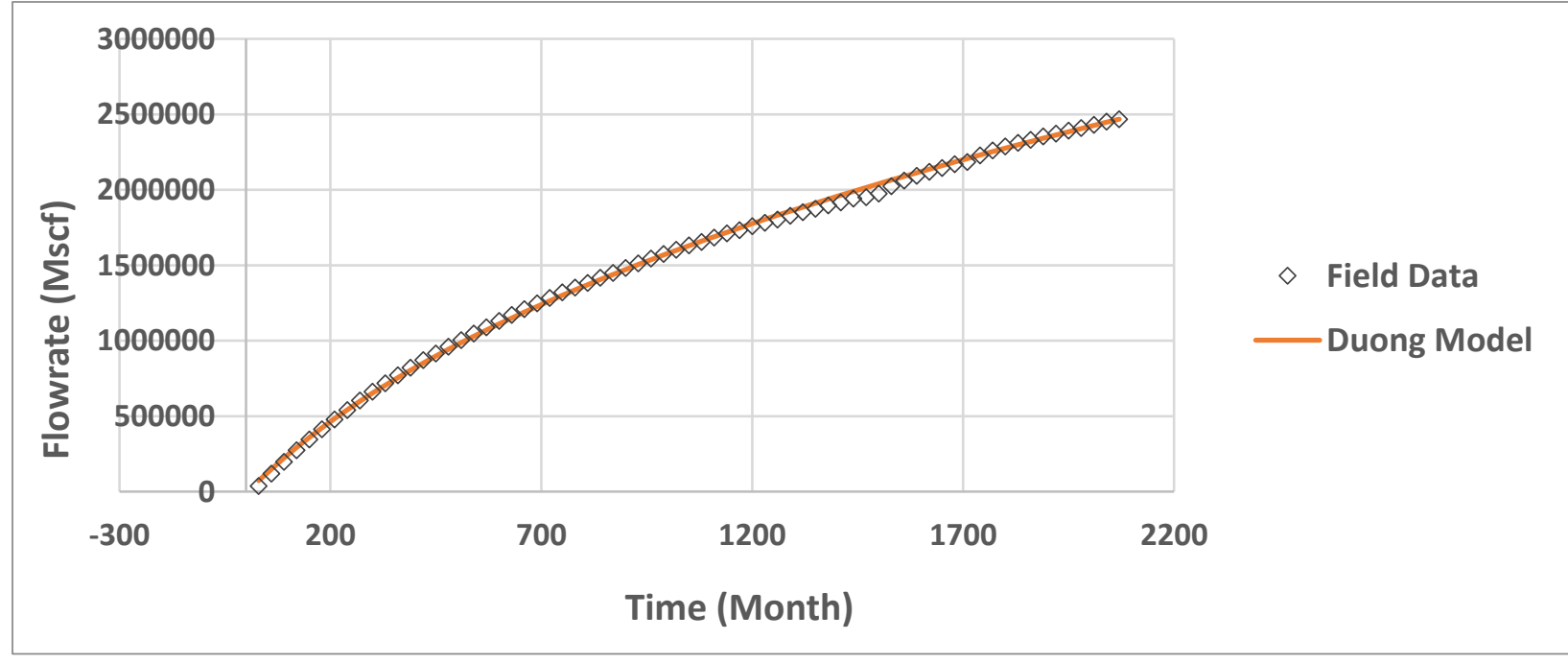

Figure 25: Comparison of Well No.4H Cumulative Production and Duong Model 
- Well No.6H

$\checkmark$ Field data: 69 months (Sample calculations)

$\underline{\text { Step 1: Calculate } q \text { and } G_{p}}$

Table 13: Well No.6H Field Data

\begin{tabular}{|c|c|c|c|c|}
\hline \multicolumn{2}{|c|}{ Given Field Data } & \multicolumn{2}{c}{} \\
\cline { 1 - 2 } \multicolumn{2}{|c|}{ Time } & Monthly & $\begin{array}{c}\text { Average } \\
\text { Daily }\end{array}$ & \multirow{2}{*}{ Cumulative } \\
\hline Month & Days & Flow Rate & Flow Rate & \\
\hline 1 & 30 & 22,174 & 739.133333 & 22174 \\
\hline 2 & 60 & 44,127 & 1470.9 & 66301 \\
\hline 3 & 90 & 42,766 & 1425.53333 & 109067 \\
\hline 4 & 120 & 42,256 & 1408.53333 & 151323 \\
\hline 5 & 150 & 39,435 & 1314.5 & 190758 \\
\hline
\end{tabular}

Step 2: Plot (q/G $\mathbf{G}_{\mathrm{p}}$ vs. time) in a $\log -\log$ plot and find a \& $\mathrm{m}$

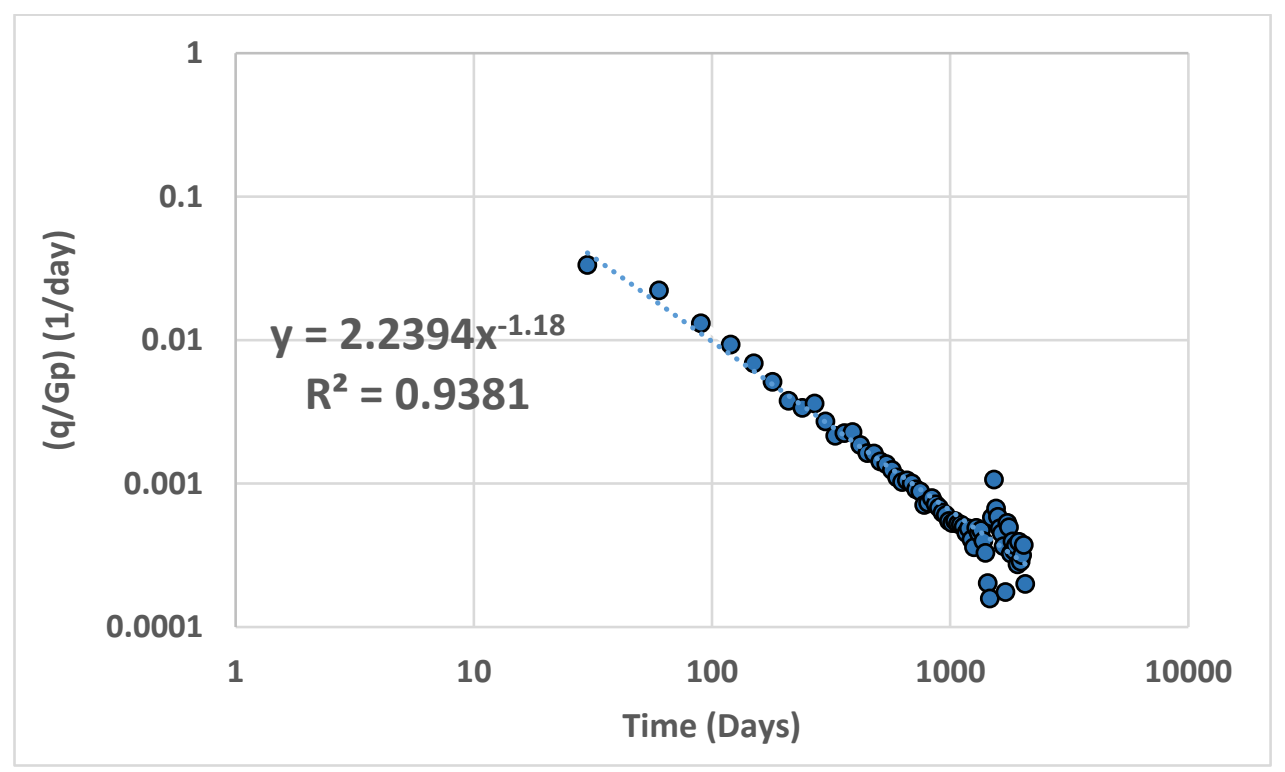

Figure 26: Well No.6H q/G $/ G_{p}$ vs. time 
Step 3: Calculate t-(a,m)

\begin{tabular}{|c|c|}
\hline$\underline{\underline{\mathbf{a}}}$ & $\underline{\underline{2.239}}$ \\
\hline$\underline{\underline{\mathbf{m}}}$ & $\underline{\underline{1.18}}$ \\
\hline
\end{tabular}

$$
t_{(a, m)}=t^{-m_{e}}\left[\frac{a}{1-m}\left(t^{\left.1-m_{-1}\right)}\right]\right.
$$

Table 14: Well No.1 t- $-(\underline{a, m})$

\begin{tabular}{|c|c|c|c|}
\hline q (Mscf) & $\begin{array}{c}\text { Gp } \\
\text { (Mscf) }\end{array}$ & $\begin{array}{c}\text { (q/Gp) } \\
(\mathbf{1} / \mathbf{d a y})\end{array}$ & $\mathbf{t}(\mathbf{a , m})$ \\
\hline 739.1333 & 22174 & 0.033333333 & 5.374966637 \\
\hline 1470.9 & 66301 & 0.022185186 & 5.232333044 \\
\hline 1425.533 & 109067 & 0.013070253 & 4.930221136 \\
\hline 1408.533 & 151323 & 0.009308125 & 4.642152034 \\
\hline 1314.5 & 190758 & 0.00689093 & 4.387398992 \\
\hline
\end{tabular}

Step 4: Plot q-vs. $\underline{t}$ (a,m) on a Cartesian graph. The straight line with the slope of qi and intercept of qळ. $\quad q=q_{1} t_{(a, m)}+q_{\infty}$

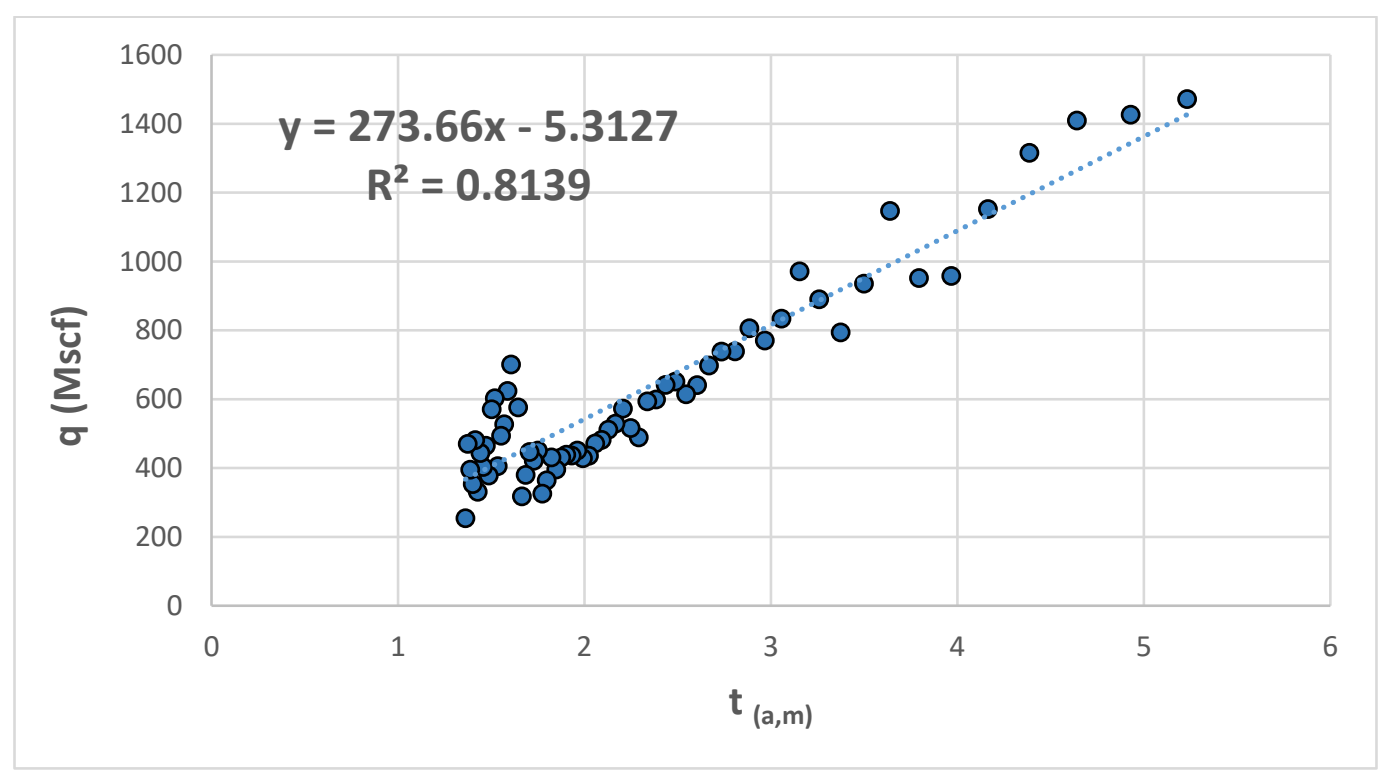

Figure 27: Well No.6H q vs. $t_{(a, m)}$ 
Where: $q=$ flow rate, volume/time

\begin{tabular}{|c|c|}
\hline $\mathbf{q i}$ & 273.66 \\
\hline $\mathbf{q} \infty$ & 5.313 \\
\hline
\end{tabular}

$a=$ Vertical axis intercept of $\log \frac{q}{G p}$ vs $t$

$m=$ slope of $\log -\log \frac{q}{G p}$ vs $t$

$G p=$ Cumulative gas production

Step 5: Gas rate forecasts can be obtained from

Table 15: Well No.6H Gp_Doung(Mscf)

\begin{tabular}{|c|c|c|c|c|c|c|c|c|c|}
\hline \multicolumn{10}{|c|}{ Given Field Data } \\
\hline \multicolumn{2}{|c|}{ Time } & \multicolumn{3}{|c|}{ Flow Rate } & \multirow[b]{2}{*}{$\begin{array}{l}(\mathbf{q} / \mathbf{G p}) \\
(\mathbf{1} / \mathbf{d a y})\end{array}$} & \multirow[b]{2}{*}{$\mathbf{t}(\mathbf{a}, \mathbf{m})$} & \multirow[b]{2}{*}{$\begin{array}{l}\text { q-Duong } \\
\text { (Mscf/d) }\end{array}$} & \multirow[b]{2}{*}{$\begin{array}{l}\text { q-Duong } \\
\text { (Mscf/m) }\end{array}$} & \multirow[b]{2}{*}{$\begin{array}{c}\text { Gp_Doung } \\
\text { (Mscf) }\end{array}$} \\
\hline Month & Days & $\begin{array}{l}\text { Monthly } \\
\text { (Mscf) }\end{array}$ & $\begin{array}{c}\text { Daily } \\
\text { (Mscf/d) }\end{array}$ & $\begin{array}{c}\text { Cum. } \\
\text { Gp } \\
\text { (Mscf) }\end{array}$ & & & & & \\
\hline 1 & 30 & 22,174 & 739.13 & 22174 & 0.033333333 & 5.374966637 & 1517.56 & 45526.89 & 45526.89 \\
\hline 2 & 60 & 44,127 & 1470.90 & 66301 & 0.022185186 & 5.232333044 & 1477.91 & 44337.20 & 89864.09 \\
\hline 3 & 90 & 42,766 & 1425.53 & 109067 & 0.013070253 & 4.930221136 & 1393.91 & 41817.31 & 131681.40 \\
\hline 4 & 120 & 42,256 & 1408.53 & 151323 & 0.009308125 & 4.642152034 & 1313.82 & 39414.56 & 171095.95 \\
\hline 5 & 150 & 39,435 & 1314.50 & 190758 & 0.00689093 & 4.387398992 & 1242.99 & 37289.69 & 208385.64 \\
\hline
\end{tabular}

\section{Well No. 6H Estimated Ultimate Recovery (EUR)}

The estimated ultimate recovery was calculated using decline curve analysis to predict production forecast for 20 and 30 years.

\begin{tabular}{|c|}
\hline EUR (BCF) for 20 years \\
\hline 2.77 \\
\hline EUR (BCF) for 30 years \\
\hline 3.20 \\
\hline
\end{tabular}




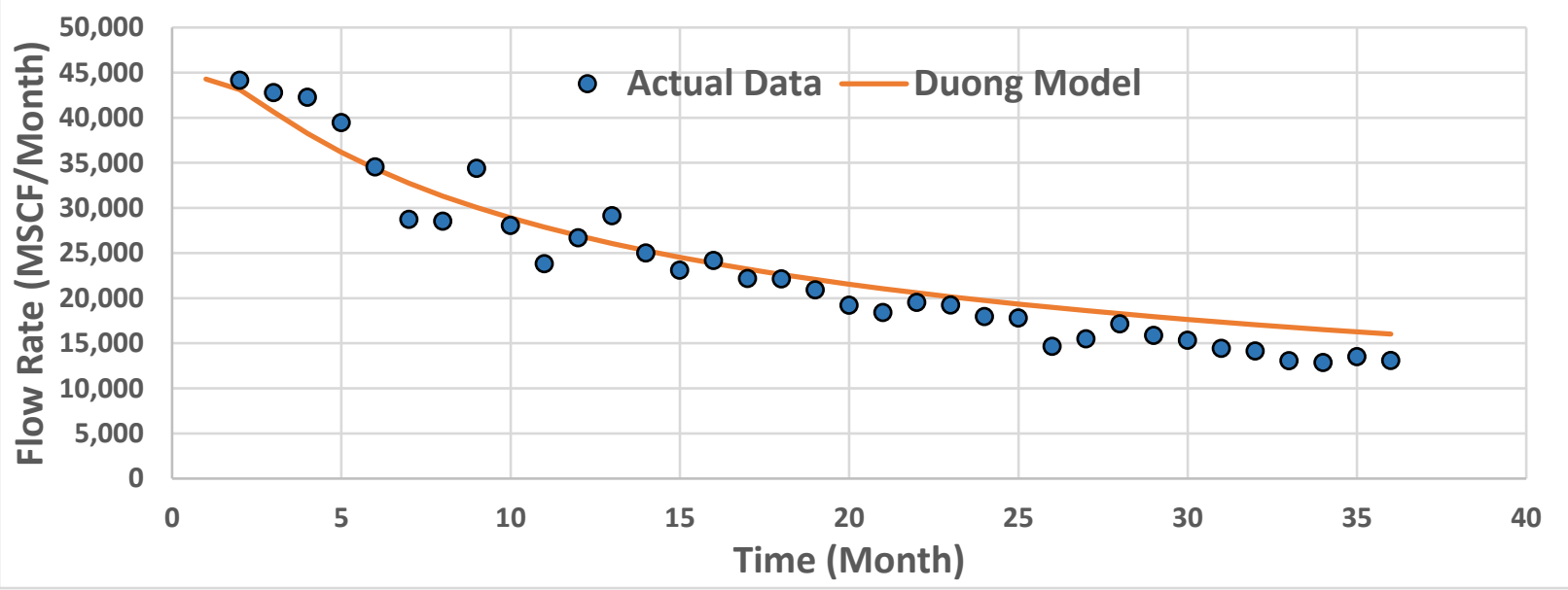

Figure 28: History Matching Well No.6H Gas Flow Rates with Duong Model

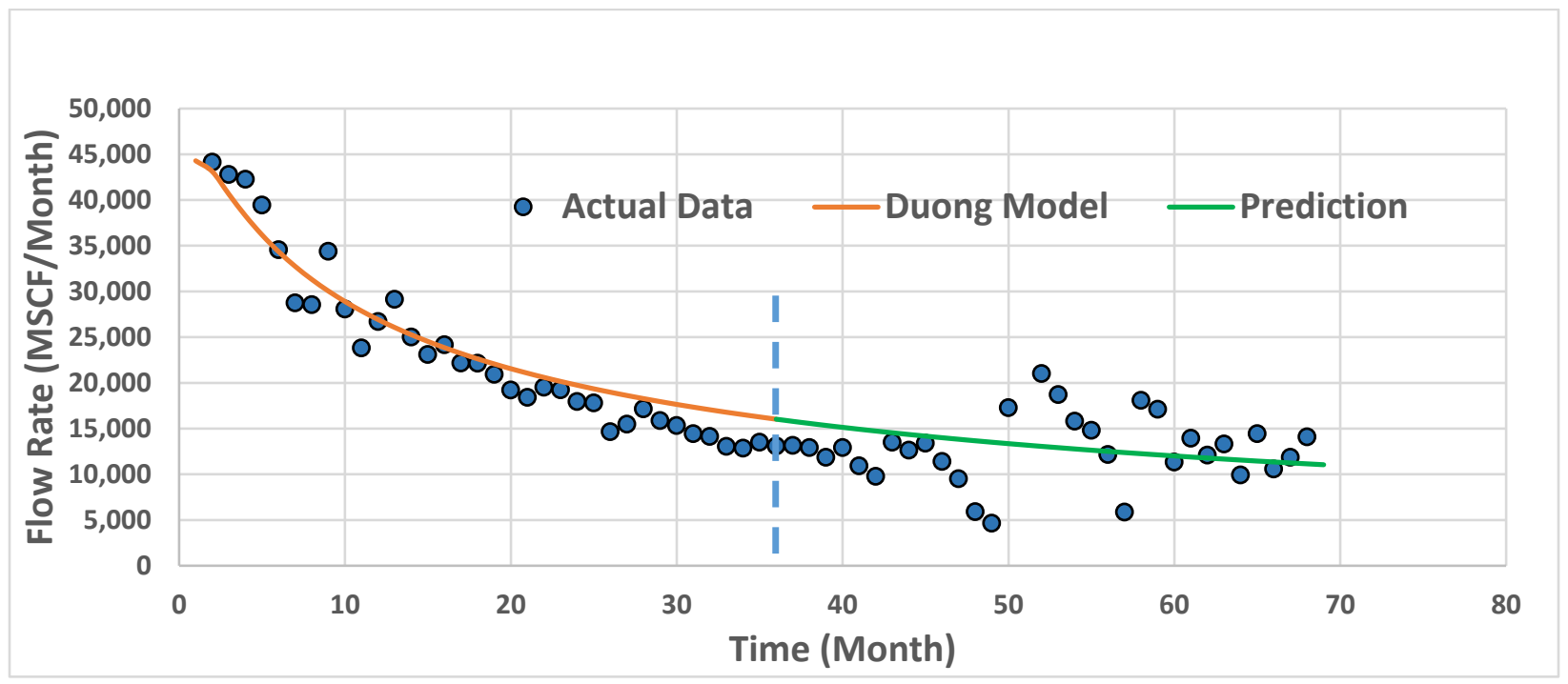

Figure 29: Comparison of the Predicted and Actual Gas Flow Rates for Well No.6H 


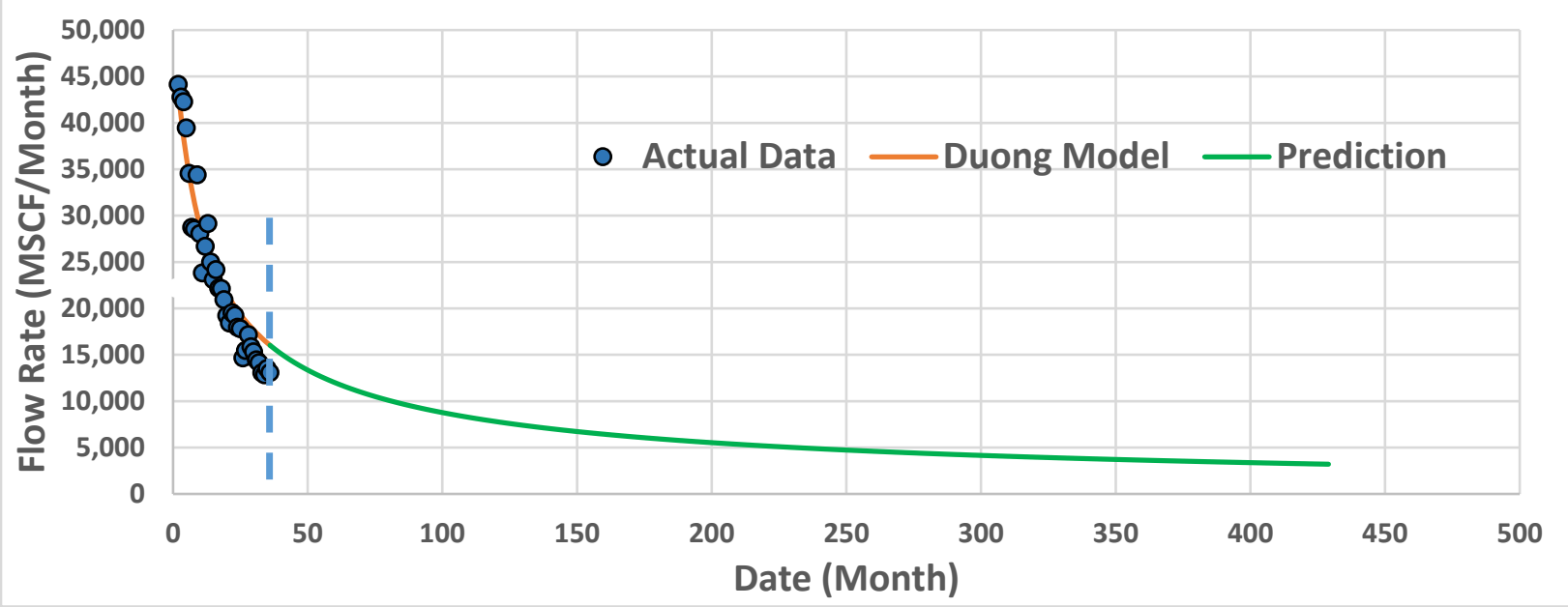

Figure 30: Predicted Gas Flow Rates for Well No.6H by Duong Model

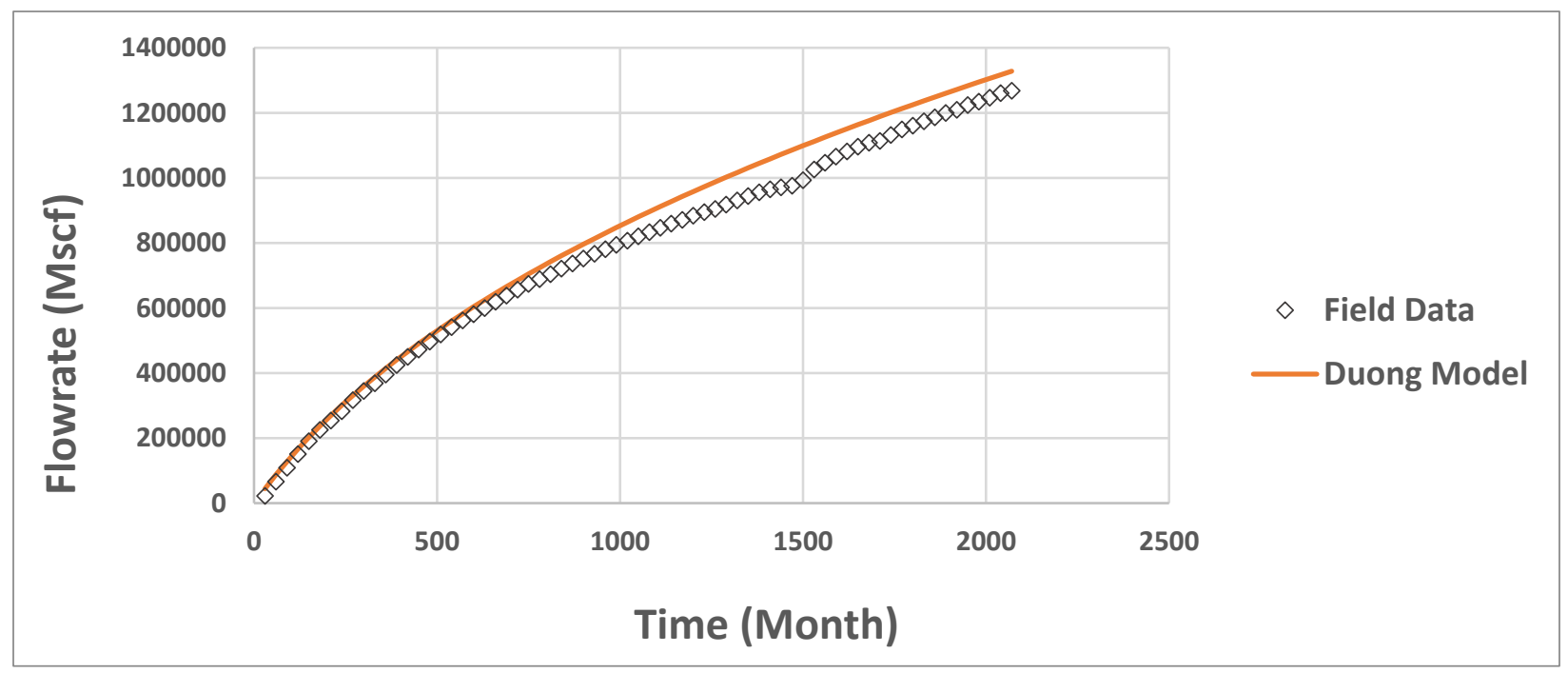

Figure 31: Comparison of Well No.6H Cumulative Production and Duong Model 
- ARP's Model Calculation \& Analysis for Well No.1

$$
\frac{1}{q} \frac{\partial q}{\partial t}=-D\left(\frac{q}{q_{i}}\right)^{b}
$$

Where: $\quad$ D: (nominal) Decline rate [t-1], month-1, year-1

b: Decline exponent, dimensionless, $0 \leq \mathrm{b} \leq 1$

qi (q0): Initial flow rate

q: flow rate at time $\mathrm{t}$

$0<\mathrm{b}<1 \longrightarrow \frac{1}{q} \frac{\partial q}{\partial t}=-D\left(\frac{q}{q_{i}}\right)^{b} \quad$ Hyperbolic Decline

$\mathrm{b}=\mathbf{0 . 5 4}$

Table 16: Well No.1 ARP's Model Calculations

\begin{tabular}{|c|c|c|c|c|c|}
\hline$b$ & 0.54 & $A v g-q$ & 877.2138211 & $A v g-G p$ & $\mathbf{6 9 3 , 0 6 6}$ \\
\hline$D i$ & $1.12 \mathrm{E}-03$ & $S S D$ & $\mathbf{5 9 1 6 8 2 . 3 4 1 4}$ & $S S D$ & $\mathbf{7 6 6 5 6 9 0 1 2 3 0}$ \\
\hline$q i$ & 1484.595661 & $S S T$ & $\mathbf{4 0 0 8 6 9 9 . 3 4 4}$ & $S S T$ & $\mathbf{3 , 9 5 8 , 6 9 8 , 1 4 1 , 6 1 6}$ \\
\hline Slope & $1.18 \mathrm{E}-05$ & $R^{2}$ & $\mathbf{8 5 . 2 4 \%}$ & $R^{2}$ & $\mathbf{9 8 . 0 6 \%}$ \\
\hline Int. & $1.94 \mathrm{E}-02$ & \multicolumn{5}{|r}{} \\
\cline { 1 - 5 }
\end{tabular}

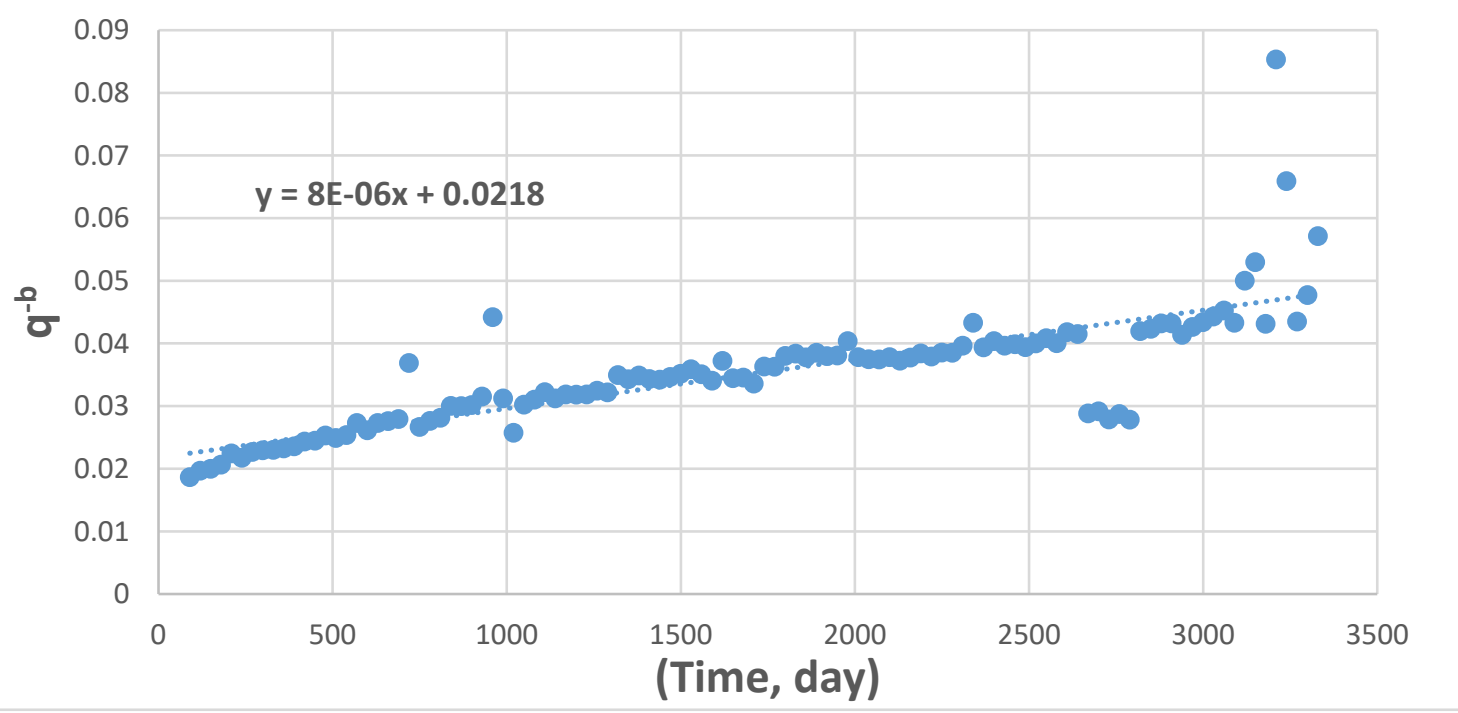

Figure 32: Arp's Model Well No.1 


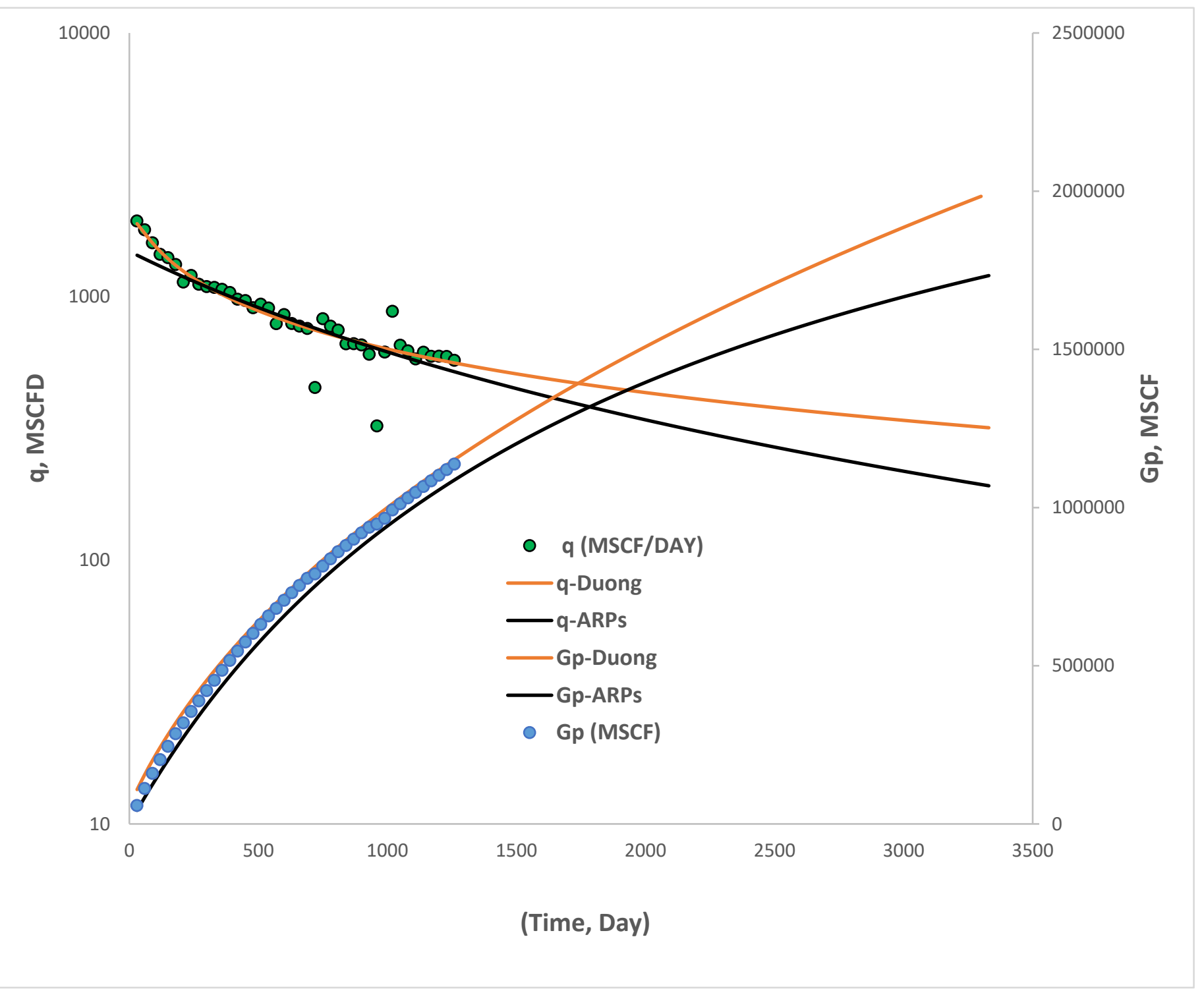

Figure 33: History Matching: Well No. 1 (ARP's Model vs. Duong Models) 


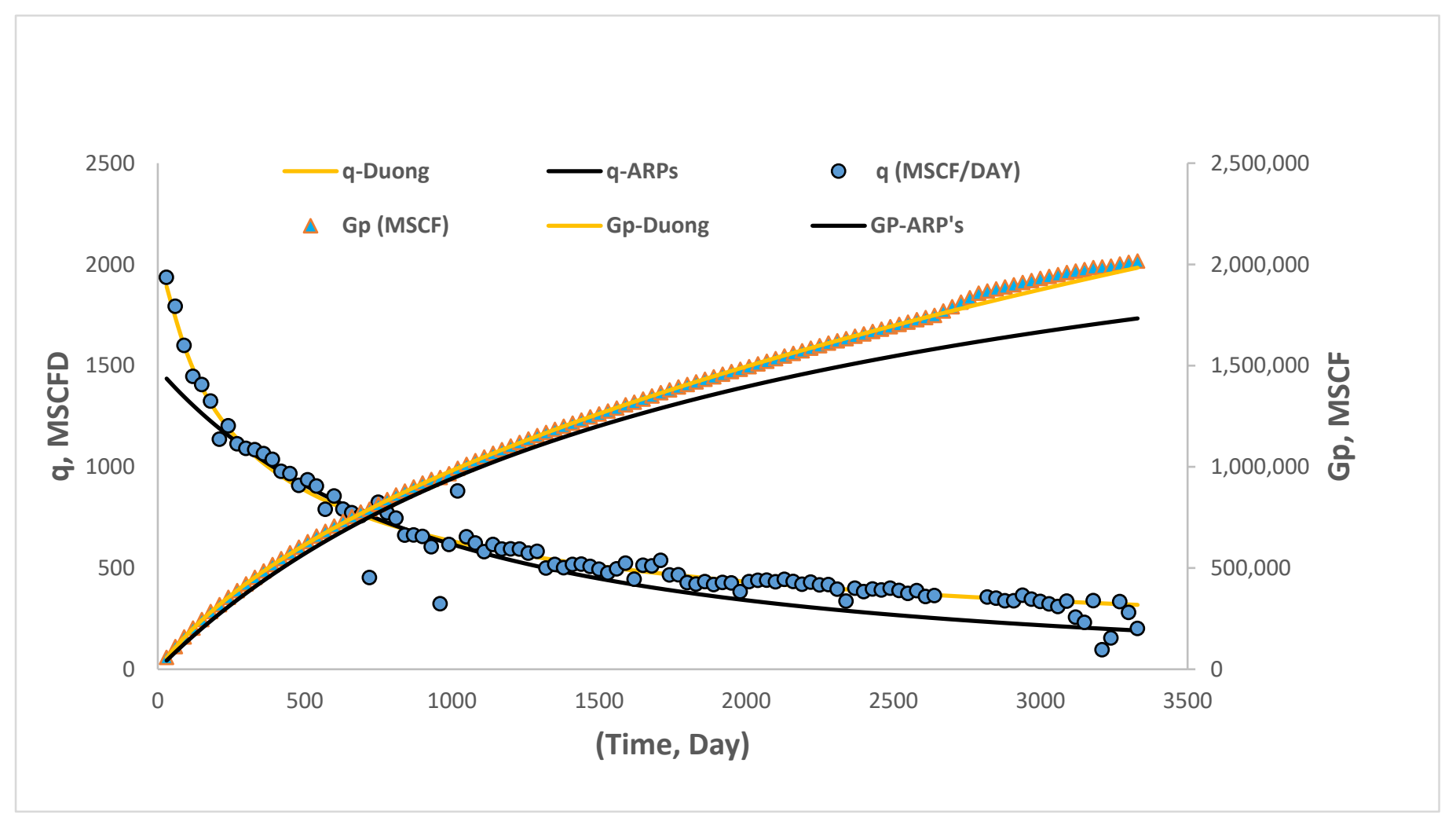

Figure 34: Comparison of the Predicted Gas Flow Rates for Well No.1 by Arp's and Duong Models 


\section{Chapter 4: Conclusions}

- Shale production analysis utilizing traditional decline curve methods will not have an accurate production forecast due to the nature of the reservoir properties and production behavior.

- Duong model is considered simple and practical to use for predicting future forecast.

- Well no.3 has the highest EUR production forecast while well no.2 has the lowest production among the 5 wells.

- The new decline curve approaches can predict future production rates for shale gas wells under transient flow.

- Arp's models considered the value of $\boldsymbol{n}$ between 0 and 1 which can be applied for conventional reservoirs under boundary dominated flow only.

- PLE decline model is developed specifically for tight gas reservoirs to match early transient data.

- The stretched exponential decline model is more complex than other models for decline curve analysis and more difficult to apply. 


\section{References}

1. Arps, J. J. (1945). Analysis of decline curves. Transactions of the AIME, 160(01), 228247.

2. Duong, A. N. (2011, June 1). Rate-Decline Analysis for Fracture-Dominated Shale Reservoirs. SPE. doi:10.2118/137748-PA

3. El sgher, M., Aminian, K., \& Ameri, S. (2018, January 23). The Impact of Stress on Propped Fracture Conductivity and Gas Recovery in Marcellus Shale. Society of Petroleum Engineers. doi:10.2118/189899-MS

4. El Sgher, M., Aminian, K., \& Ameri, S. (2018, April 22). Geomechanical Impact on Gas Recovery from Marcellus Shale. Society of Petroleum Engineers. doi:10.2118/190054-MS

5. Ilk, D. et. al. 2008. Exponential vs. Hyperbolic Decline in Tight Gas Sands: Understanding the Origin and Implications for Reserve Estimates Using Arps' Decline Curves. Paper 116731-MS presented at the SPE Annual Technical Conference and Exhibition, Denver, Colorado, USA, 21-24 September 2008. DOI: 10.2118/116731-MS.

6. Joshi, K., \& Lee, W. J. (2013, February 4). Comparison of Various Deterministic Forecasting Techniques in Shale Gas Reservoirs. Society of Petroleum Engineers. doi:10.2118/163870-MS

7. Seshadri, Jagan Nathan, and Louis Mattar. "Comparison of power law and modified hyperbolic decline methods." In Canadian Unconventional Resources and International Petroleum Conference. Society of Petroleum Engineers, 2010.

8. Valko, P. P., \& Lee, W. J. (2010, January 1). A Better Way to Forecast Production from Unconventional GasWells. Society of Petroleum Engineers. doi:10.2118/134231-MS

9. Vanorsdale, C., 2013. Production Decline Analysis Lessons from Classic Shale Gas Wells. Paper SPE 166205 Presented at the 2013 SPE Annual Technical Conference and Exhibition, New Orleans, LA, USA, 30 September 2 October. SPE-177300-MS 31 


\section{Appendix}

Well No. 1 Calculations (Duong Model)

\begin{tabular}{|c|c|c|c|c|c|c|c|c|c|}
\hline \multicolumn{2}{|c|}{ Time } & \multirow{2}{*}{$\begin{array}{l}\text { Flow Rate } \\
\text { Mscf/Month }\end{array}$} & \multirow[b]{2}{*}{$\underset{(M s c f / d)}{q}$} & \multirow[b]{2}{*}{$\begin{array}{c}\text { Gp } \\
\text { (Mscf) }\end{array}$} & \multirow[b]{2}{*}{$\begin{array}{l}\text { (q/Gp) } \\
\text { (1/day) }\end{array}$} & \multirow[b]{2}{*}{$t(a, m)$} & \multirow[b]{2}{*}{$\begin{array}{l}\text { q-Duong } \\
\text { (Mscf/d) }\end{array}$} & \multirow[b]{2}{*}{$\begin{array}{l}\text { q-Duong } \\
\text { (Mscf/m) }\end{array}$} & \multirow[b]{2}{*}{$\begin{array}{c}\text { Gp_Doung } \\
\text { (Mscf) }\end{array}$} \\
\hline Month & Days & & & & & & & & \\
\hline 1 & 30 & 58056 & 1935.20 & 58056 & 0.0333 & 2.524 & 1882 & 56474 & 56474 \\
\hline 2 & 60 & 53757 & 1791.90 & 111813 & 0.0160 & 2.319 & 1731 & 51930 & 108404 \\
\hline 3 & 90 & 47962 & 1598.73 & 159775 & 0.0100 & 2.133 & 1594 & 47820 & 156224 \\
\hline 4 & 120 & 43370 & 1445.67 & 203145 & 0.0071 & 1.982 & 1483 & 44482 & 200706 \\
\hline 5 & 150 & 42172 & 1405.73 & 245317 & 0.0057 & 1.859 & 1391 & 41737 & 242443 \\
\hline 6 & 180 & 39718 & 1323.93 & 285035 & 0.0046 & 1.755 & 1315 & 39435 & 281878 \\
\hline 7 & 210 & 34057 & 1135.23 & 319092 & 0.0036 & 1.666 & 1249 & 37468 & 319347 \\
\hline 8 & 240 & 36048 & 1201.60 & 355140 & 0.0034 & 1.589 & 1192 & 35761 & 355108 \\
\hline 9 & 270 & 33385 & 1112.83 & 388525 & 0.0029 & 1.521 & 1142 & 34261 & 389369 \\
\hline 10 & 300 & 32712 & 1090.40 & 421237 & 0.0026 & 1.461 & 1098 & 32928 & 422296 \\
\hline 11 & 330 & 32507 & 1083.57 & 453744 & 0.0024 & 1.407 & 1058 & 31732 & 454028 \\
\hline 12 & 360 & 31938 & 1064.60 & 485682 & 0.0022 & 1.358 & 1022 & 30652 & 484680 \\
\hline 13 & 390 & 31091 & 1036.37 & 516773 & 0.0020 & 1.314 & 989 & 29669 & 514349 \\
\hline 14 & 420 & 29312 & 977.07 & 546085 & 0.0018 & 1.273 & 959 & 28770 & 543119 \\
\hline 15 & 450 & 28978 & 965.93 & 575063 & 0.0017 & 1.236 & 931 & 27942 & 571062 \\
\hline 16 & 480 & 27224 & 907.47 & 602287 & 0.0015 & 1.202 & 906 & 27178 & 598239 \\
\hline 17 & 510 & 28059 & 935.30 & 630346 & 0.0015 & 1.170 & 882 & 26468 & 624708 \\
\hline 18 & 540 & 27138 & 904.60 & 657484 & 0.0014 & 1.140 & 860 & 25808 & 650516 \\
\hline 19 & 570 & 23676 & 789.20 & 681160 & 0.0012 & 1.112 & 840 & 25190 & 675706 \\
\hline 20 & 600 & 25641 & 854.70 & 706801 & 0.0012 & 1.086 & 820 & 24612 & 700317 \\
\hline 21 & 630 & 23698 & 789.93 & 730499 & 0.0011 & 1.061 & 802 & 24068 & 724385 \\
\hline 22 & 660 & 23181 & 772.70 & 753680 & 0.0010 & 1.038 & 785 & 23555 & 747941 \\
\hline 23 & 690 & 22711 & 757.03 & 776391 & 0.0010 & 1.016 & 769 & 23072 & 771012 \\
\hline 24 & 720 & 13557 & 451.90 & 789948 & 0.0006 & 0.996 & 754 & 22614 & 793626 \\
\hline 25 & 750 & 24718 & 823.93 & 814666 & 0.0010 & 0.976 & 739 & 22179 & 815805 \\
\hline 26 & 780 & 23170 & 772.33 & 837836 & 0.0009 & 0.957 & 726 & 21767 & 837572 \\
\hline 27 & 810 & 22370 & 745.67 & 860206 & 0.0009 & 0.940 & 712 & 21374 & 858946 \\
\hline 28 & 840 & 19851 & 661.70 & 880057 & 0.0008 & 0.923 & 700 & 21000 & 879946 \\
\hline 29 & 870 & 19879 & 662.63 & 899936 & 0.0007 & 0.907 & 688 & 20643 & 900588 \\
\hline 30 & 900 & 19667 & 655.57 & 919603 & 0.0007 & 0.891 & 677 & 20301 & 920889 \\
\hline 31 & 930 & 18131 & 604.37 & 937734 & 0.0006 & 0.876 & 666 & 19974 & 940864 \\
\hline 32 & 960 & 9692 & 323.07 & 947426 & 0.0003 & 0.862 & 655 & 19661 & 960525 \\
\hline
\end{tabular}




\begin{tabular}{|c|c|c|c|c|c|c|c|c|c|}
\hline 33 & 990 & 18468 & 615.60 & 965894 & 0.0006 & 0.849 & 645 & 19361 & 979885 \\
\hline 34 & 1020 & 26389 & 879.63 & 992283 & 0.0009 & 0.836 & 636 & 19072 & 998957 \\
\hline 35 & 1050 & 19614 & 653.80 & 1011897 & 0.0006 & 0.823 & 626 & 18794 & 1017751 \\
\hline 36 & 1080 & 18682 & 622.73 & 1030579 & 0.0006 & 0.811 & 618 & 18527 & 1036278 \\
\hline 37 & 1110 & 17402 & 580.07 & 1047981 & 0.0006 & 0.800 & 609 & 18270 & 1054548 \\
\hline 38 & 1140 & 18456 & 615.20 & 1066437 & 0.0006 & 0.788 & 601 & 18021 & 1072570 \\
\hline 39 & 1170 & 17796 & 593.20 & 1084233 & 0.0005 & 0.778 & 593 & 17782 & 1090352 \\
\hline 40 & 1200 & 17819 & 593.97 & 1102052 & 0.0005 & 0.767 & 585 & 17551 & 1107902 \\
\hline 41 & 1230 & 17786 & 592.87 & 1119838 & 0.0005 & 0.757 & 578 & 17327 & 1125229 \\
\hline 42 & 1260 & 17191 & 573.03 & 1137029 & 0.0005 & 0.747 & 570 & 17111 & 1142340 \\
\hline 43 & 1290 & 17449 & 581.63 & 1154478 & 0.0005 & 0.738 & 563 & 16901 & 1159241 \\
\hline 44 & 1320 & 14965 & 498.83 & 1169443 & 0.0004 & 0.729 & 557 & 16698 & 1175939 \\
\hline 45 & 1350 & 15517 & 517.23 & 1184960 & 0.0004 & 0.720 & 550 & 16502 & 1192441 \\
\hline 46 & 1380 & 15034 & 501.13 & 1199994 & 0.0004 & 0.711 & 544 & 16311 & 1208752 \\
\hline 47 & 1410 & 15497 & 516.57 & 1215491 & 0.0004 & 0.703 & 538 & 16126 & 1224878 \\
\hline 48 & 1440 & 15564 & 518.80 & 1231055 & 0.0004 & 0.695 & 532 & 15946 & 1240825 \\
\hline 49 & 1470 & 15228 & 507.60 & 1246283 & 0.0004 & 0.687 & 526 & 15772 & 1256597 \\
\hline 50 & 1500 & 14806 & 493.53 & 1261089 & 0.0004 & 0.679 & 520 & 15602 & 1272199 \\
\hline 51 & 1530 & 14252 & 475.07 & 1275341 & 0.0004 & 0.672 & 515 & 15437 & 1287636 \\
\hline 52 & 1560 & 14863 & 495.43 & 1290204 & 0.0004 & 0.664 & 509 & 15277 & 1302912 \\
\hline 53 & 1590 & 15708 & 523.60 & 1305912 & 0.0004 & 0.657 & 504 & 15120 & 1318033 \\
\hline 54 & 1620 & 13326 & 444.20 & 1319238 & 0.0003 & 0.651 & 499 & 14968 & 1333001 \\
\hline 55 & 1650 & 15377 & 512.57 & 1334615 & 0.0004 & 0.644 & 494 & 14820 & 1347820 \\
\hline 56 & 1680 & 15286 & 509.53 & 1349901 & 0.0004 & 0.637 & 489 & 14675 & 1362495 \\
\hline 57 & 1710 & 16111 & 537.03 & 1366012 & 0.0004 & 0.631 & 484 & 14534 & 1377030 \\
\hline 58 & 1740 & 13951 & 465.03 & 1379963 & 0.0003 & 0.625 & 480 & 14397 & 1391426 \\
\hline 59 & 1770 & 13975 & 465.83 & 1393938 & 0.0003 & 0.619 & 475 & 14262 & 1405688 \\
\hline 60 & 1800 & 12817 & 427.23 & 1406755 & 0.0003 & 0.613 & 471 & 14131 & 1419819 \\
\hline 61 & 1830 & 12595 & 419.83 & 1419350 & 0.0003 & 0.607 & 467 & 14003 & 1433823 \\
\hline 62 & 1860 & 12957 & 431.90 & 1432307 & 0.0003 & 0.601 & 463 & 13878 & 1447701 \\
\hline 63 & 1890 & 12512 & 417.07 & 1444819 & 0.0003 & 0.596 & 459 & 13756 & 1461457 \\
\hline 64 & 1920 & 12833 & 427.77 & 1457652 & 0.0003 & 0.590 & 455 & 13636 & 1475093 \\
\hline 65 & 1950 & 12768 & 425.60 & 1470420 & 0.0003 & 0.585 & 451 & 13520 & 1488612 \\
\hline 66 & 1980 & 11468 & 382.27 & 1481888 & 0.0003 & 0.580 & 447 & 13405 & 1502018 \\
\hline 67 & 2010 & 12951 & 431.70 & 1494839 & 0.0003 & 0.575 & 443 & 13293 & 1515311 \\
\hline 68 & 2040 & 13154 & 438.47 & 1507993 & 0.0003 & 0.570 & 439 & 13184 & 1528495 \\
\hline 69 & 2070 & 13186 & 439.53 & 1521179 & 0.0003 & 0.565 & 436 & 13077 & 1541571 \\
\hline 70 & 2100 & 12946 & 431.53 & 1534125 & 0.0003 & 0.560 & 432 & 12972 & 1554543 \\
\hline 71 & 2130 & 13311 & 443.70 & 1547436 & 0.0003 & 0.556 & 429 & 12869 & 1567412 \\
\hline 72 & 2160 & 12993 & 433.10 & 1560429 & 0.0003 & 0.551 & 426 & 12768 & 1580179 \\
\hline
\end{tabular}




\begin{tabular}{|c|c|c|c|c|c|c|c|c|c|}
\hline 73 & 2190 & 12568 & 418.93 & 1572997 & 0.0003 & 0.547 & 422 & 12669 & 1592848 \\
\hline 74 & 2220 & 12885 & 429.50 & 1585882 & 0.0003 & 0.542 & 419 & 12572 & 1605421 \\
\hline 75 & 2250 & 12484 & 416.13 & 1598366 & 0.0003 & 0.538 & 416 & 12477 & 1617898 \\
\hline 76 & 2280 & 12511 & 417.03 & 1610877 & 0.0003 & 0.534 & 413 & 12384 & 1630282 \\
\hline 77 & 2310 & 11852 & 395.07 & 1622729 & 0.0002 & 0.530 & 410 & 12293 & 1642574 \\
\hline 78 & 2340 & 10063 & 335.43 & 1632792 & 0.0002 & 0.526 & 407 & 12203 & 1654777 \\
\hline 79 & 2370 & 12000 & 400.00 & 1644792 & 0.0002 & 0.522 & 404 & 12115 & 1666892 \\
\hline 80 & 2400 & 11469 & 382.30 & 1656261 & 0.0002 & 0.518 & 401 & 12028 & 1678920 \\
\hline 81 & 2430 & 11858 & 395.27 & 1668119 & 0.0002 & 0.514 & 398 & 11943 & 1690863 \\
\hline 82 & 2460 & 11731 & 391.03 & 1679850 & 0.0002 & 0.510 & 395 & 11860 & 1702723 \\
\hline 83 & 2490 & 11994 & 399.80 & 1691844 & 0.0002 & 0.507 & 393 & 11778 & 1714501 \\
\hline 84 & 2520 & 11661 & 388.70 & 1703505 & 0.0002 & 0.503 & 390 & 11697 & 1726199 \\
\hline 85 & 2550 & 11221 & 374.03 & 1714726 & 0.0002 & 0.499 & 387 & 11618 & 1737817 \\
\hline 86 & 2580 & 11637 & 387.90 & 1726363 & 0.0002 & 0.496 & 385 & 11541 & 1749358 \\
\hline 87 & 2610 & 10746 & 358.20 & 1737109 & 0.0002 & 0.492 & 382 & 11464 & 1760822 \\
\hline 88 & 2640 & 10886 & 362.87 & 1747995 & 0.0002 & 0.489 & 380 & 11389 & 1772211 \\
\hline 89 & 2670 & 21386 & 712.87 & 1769381 & 0.0004 & 0.486 & 377 & 11315 & 1783526 \\
\hline 90 & 2700 & 20962 & 698.73 & 1790343 & 0.0004 & 0.482 & 375 & 11242 & 1794768 \\
\hline 91 & 2730 & 22794 & 759.80 & 1813137 & 0.0004 & 0.479 & 372 & 11171 & 1805939 \\
\hline 92 & 2760 & 21550 & 718.33 & 1834687 & 0.0004 & 0.476 & 370 & 11100 & 1817040 \\
\hline 93 & 2790 & 22830 & 761.00 & 1857517 & 0.0004 & 0.473 & 368 & 11031 & 1828071 \\
\hline 94 & 2820 & 10674 & 355.80 & 1868191 & 0.0002 & 0.470 & 365 & 10963 & 1839034 \\
\hline 95 & 2850 & 10503 & 350.10 & 1878694 & 0.0002 & 0.467 & 363 & 10896 & 1849929 \\
\hline 96 & 2880 & 10111 & 337.03 & 1888805 & 0.0002 & 0.464 & 361 & 10830 & 1860759 \\
\hline 97 & 2910 & 10111 & 337.03 & 1898916 & 0.0002 & 0.461 & 359 & 10765 & 1871524 \\
\hline 98 & 2940 & 10951 & 365.03 & 1909867 & 0.0002 & 0.458 & 357 & 10701 & 1882224 \\
\hline 99 & 2970 & 10357 & 345.23 & 1920224 & 0.0002 & 0.455 & 355 & 10637 & 1892862 \\
\hline 100 & 3000 & 10025 & 334.17 & 1930249 & 0.0002 & 0.452 & 353 & 10575 & 1903437 \\
\hline 101 & 3030 & 9657 & 321.90 & 1939906 & 0.0002 & 0.450 & 350 & 10514 & 1913951 \\
\hline 102 & 3060 & 9270 & 309.00 & 1949176 & 0.0002 & 0.447 & 348 & 10454 & 1924405 \\
\hline 103 & 3090 & 10061 & 335.37 & 1959237 & 0.0002 & 0.444 & 346 & 10394 & 1934799 \\
\hline 104 & 3120 & 7703 & 256.77 & 1966940 & 0.0001 & 0.441 & 345 & 10335 & 1945134 \\
\hline 105 & 3150 & 6926 & 230.87 & 1973866 & 0.0001 & 0.439 & 343 & 10278 & 1955412 \\
\hline 106 & 3180 & 10142 & 338.07 & 1984008 & 0.0002 & 0.436 & 341 & 10221 & 1965632 \\
\hline 107 & 3210 & 2864 & 95.47 & 1986872 & 0.0000 & 0.434 & 339 & 10164 & 1975797 \\
\hline 108 & 3240 & 4619 & 153.97 & 1991491 & 0.0001 & 0.431 & 337 & 10109 & 1985905 \\
\hline 109 & 3270 & 9989 & 332.97 & 2001480 & 0.0002 & 0.429 & 335 & 10054 & 1995960 \\
\hline 110 & 3300 & 8405 & 280.17 & 2009885 & 0.0001 & 0.426 & 333 & 10000 & 2005960 \\
\hline 111 & 3330 & 6020 & 200.67 & 2015905 & 0.0001 & 0.424 & 332 & 9947 & 2015907 \\
\hline
\end{tabular}


Well No. 2 Calculations (Duong Model)

\begin{tabular}{|c|c|c|c|c|c|c|c|c|c|}
\hline \multicolumn{2}{|c|}{ Time } & \multirow{2}{*}{$\begin{array}{c}\text { Flow Rate } \\
\text { Mscf/Month }\end{array}$} & \multirow[b]{2}{*}{$\begin{array}{c}q \\
\text { (Mscf/d) }\end{array}$} & \multirow[b]{2}{*}{$\begin{array}{c}\text { Gp } \\
\text { (Mscf) }\end{array}$} & \multirow[b]{2}{*}{$\begin{array}{l}\text { (q/Gp) } \\
\text { (1/day) }\end{array}$} & \multirow[b]{2}{*}{$t(a, m)$} & \multirow[b]{2}{*}{$\begin{array}{l}\text { q-Duong } \\
\text { (Mscf/d) }\end{array}$} & \multirow[b]{2}{*}{$\begin{array}{l}\text { q-Duong } \\
\text { (Mscf/m) }\end{array}$} & \multirow[b]{2}{*}{$\begin{array}{c}\text { Gp_Doung } \\
\text { (Mscf) }\end{array}$} \\
\hline Month & Days & & & & & & & & \\
\hline 1 & 30 & 13662 & 455.40 & 13662 & 0.0333 & 10.3885 & 1167.5507 & 35026.5212 & 35026.5212 \\
\hline 2 & 60 & 44888 & 1496.27 & 58550 & 0.0256 & 10.4560 & 1175.0533 & 35251.5991 & 70278.1204 \\
\hline 3 & 90 & 38747 & 1291.57 & 97297 & 0.0133 & 9.9513 & 1118.9513 & 33568.5379 & 103846.6583 \\
\hline 4 & 120 & 36641 & 1221.37 & 133938 & 0.0091 & 9.4023 & 1057.9188 & 31737.5637 & 135584.2219 \\
\hline 5 & 150 & 32207 & 1073.57 & 166145 & 0.0065 & 8.8935 & 1001.3554 & 30040.6628 & 165624.8848 \\
\hline 6 & 180 & 30926 & 1030.87 & 197071 & 0.0052 & 8.4375 & 950.6598 & 28519.7928 & 194144.6775 \\
\hline 7 & 210 & 28869 & 962.30 & 225940 & 0.0043 & 8.0312 & 905.4924 & 27164.7728 & 221309.4504 \\
\hline 8 & 240 & 24773 & 825.77 & 250713 & 0.0033 & 7.6684 & 865.1607 & 25954.8210 & 247264.2714 \\
\hline 9 & 270 & 26040 & 868.00 & 276753 & 0.0031 & 7.3429 & 828.9722 & 24869.1646 & 272133.4360 \\
\hline 10 & 300 & 23876 & 795.87 & 300629 & 0.0026 & 7.0492 & 796.3211 & 23889.6330 & 296023.0689 \\
\hline 11 & 330 & 24049 & 801.63 & 324678 & 0.0025 & 6.7828 & 766.6992 & 23000.9774 & 319024.0463 \\
\hline 12 & 360 & 22355 & 745.17 & 347033 & 0.0021 & 6.5398 & 739.6854 & 22190.5618 & 341214.6081 \\
\hline 13 & 390 & 22628 & 754.27 & 369661 & 0.0020 & 6.3171 & 714.9310 & 21447.9303 & 362662.5384 \\
\hline 14 & 420 & 21671 & 722.37 & 391332 & 0.0018 & 6.1121 & 692.1467 & 20764.4010 & 383426.9394 \\
\hline 15 & 450 & 20383 & 679.43 & 411715 & 0.0017 & 5.9227 & 671.0908 & 20132.7250 & 403559.6644 \\
\hline 16 & 480 & 20220 & 674.00 & 431935 & 0.0016 & 5.7471 & 651.5604 & 19546.8112 & 423106.4757 \\
\hline 17 & 510 & 18870 & 629.00 & 450805 & 0.0014 & 5.5836 & 633.3836 & 19001.5071 & 442107.9828 \\
\hline 18 & 540 & 19271 & 642.37 & 470076 & 0.0014 & 5.4309 & 616.4142 & 18492.4258 & 460600.4087 \\
\hline 19 & 570 & 18656 & 621.87 & 488732 & 0.0013 & 5.2880 & & 18015.8086 & 478616.2173 \\
\hline 20 & 600 & 16235 & 541.17 & 504967 & 0.0011 & 5.1539 & 585.6139 & 17568.4156 & 496184.6328 \\
\hline 21 & 630 & 17690 & 589.67 & 522657 & 0.0011 & 5.0276 & 571.5813 & 17147.4384 & 513332.0713 \\
\hline 22 & 660 & 16727 & 557.57 & 539384 & 0.0010 & 4.9086 & 558.3477 & 16750.4297 & 530082.5009 \\
\hline 23 & 690 & 17156 & 571.87 & 556540 & 0.0010 & 4.7961 & 545.8415 & 16375.2460 & 546457.7469 \\
\hline 24 & 720 & 15455 & 515.17 & 571995 & 0.0009 & 4.6896 & 534.0000 & 16020.0014 & 562477.7483 \\
\hline 25 & 750 & 9025 & 300.83 & 581020 & 0.0005 & 4.5885 & 522.7676 & 15683.0290 & 578160.7773 \\
\hline 26 & 780 & 17522 & 584.07 & 598542 & 0.0010 & 4.4925 & 512.0950 & 15362.8498 & 593523.6271 \\
\hline 27 & 810 & 15542 & 518.07 & 614084 & 0.0008 & 4.4012 & 501.9382 & 15058.1460 & 608581.7731 \\
\hline 28 & 840 & 16045 & 534.83 & 630129 & 0.0008 & 4.3141 & 492.2580 & 14767.7395 & 623349.5125 \\
\hline 29 & 870 & 15087 & 502.90 & 645216 & 0.0008 & 4.2310 & 483.0191 & 14490.5734 & 637840.0859 \\
\hline 30 & 900 & 15673 & 522.43 & 660889 & 0.0008 & 4.1516 & 474.1899 & 14225.6963 & 652065.7823 \\
\hline 31 & 930 & 15,215 & 507.17 & 676104 & 0.0008 & 4.0756 & 465.7417 & 13972.2496 & 666038.0318 \\
\hline 32 & 960 & 13,311 & 443.70 & 689415 & 0.0006 & 4.0028 & 457.6485 & 13729.4555 & 679767.4874 \\
\hline 33 & 990 & 10,199 & 339.97 & 699614 & 0.0005 & 3.9330 & 449.8869 & 13496.6083 & 693264.0957 \\
\hline 34 & 1020 & 10,204 & 340.13 & 709818 & 0.0005 & 3.8659 & 442.4355 & 13273.0655 & 706537.1611 \\
\hline
\end{tabular}




\begin{tabular}{|c|c|c|c|c|c|c|c|c|c|}
\hline 35 & 1050 & 10,721 & 357.37 & 720539 & 0.0005 & 3.8015 & 435.2747 & 13058.2407 & 719595.4019 \\
\hline 36 & 1080 & 11,510 & 383.67 & 732049 & 0.0005 & 3.7396 & 428.3866 & 12851.5981 & 732447.0000 \\
\hline 37 & 1110 & 12,280 & 409.33 & 744329 & 0.0005 & 3.6799 & 421.7549 & 12652.6461 & 745099.6461 \\
\hline 38 & 1140 & 11,711 & 390.37 & 756040 & 0.0005 & 3.6224 & 415.3645 & 12460.9336 & 757560.5797 \\
\hline 39 & 1170 & 11,076 & 369.20 & 767116 & 0.0005 & 3.5670 & 409.2015 & 12276.0452 & 769836.6249 \\
\hline 40 & 1200 & 11,404 & 380.13 & 778520 & 0.0005 & 3.5135 & 403.2533 & 12097.5979 & 781934.2228 \\
\hline 41 & 1230 & 10,900 & 363.33 & 789420 & 0.0005 & 3.4618 & 397.5079 & 11925.2380 & 793859.4608 \\
\hline 42 & 1260 & 11,100 & 370.00 & 800520 & 0.0005 & 3.4118 & 391.9546 & 11758.6382 & 805618.0990 \\
\hline 43 & 1290 & 10,443 & 348.10 & 810963 & 0.0004 & 3.3635 & 386.5832 & 11597.4951 & 817215.5940 \\
\hline 44 & 1320 & 9,999 & 333.30 & 820962 & 0.0004 & 3.3168 & 381.3842 & 11441.5269 & 828657.1210 \\
\hline 45 & 1350 & 11,114 & 370.47 & 832076 & 0.0004 & 3.2715 & 376.3491 & 11290.4719 & 839947.5929 \\
\hline 46 & 1380 & 9,934 & 331.13 & 842010 & 0.0004 & 3.2276 & 371.4695 & 11144.0861 & 851091.6790 \\
\hline 47 & 1410 & 10,182 & 339.40 & 852192 & 0.0004 & 3.1850 & 366.7381 & 11002.1422 & 862093.8212 \\
\hline 48 & 1440 & 9,791 & 326.37 & 861983 & 0.0004 & 3.1437 & 362.1476 & 10864.4278 & 872958.2490 \\
\hline 49 & 1470 & 10,037 & 334.57 & 872020 & 0.0004 & 3.1036 & 357.6915 & 10730.7444 & 883688.9934 \\
\hline 50 & 1500 & 9,934 & 331.13 & 881954 & 0.0004 & 3.0647 & 353.3635 & 10600.9062 & 894289.8997 \\
\hline 51 & 1530 & 9,527 & 317.57 & 891481 & 0.0004 & 3.0269 & 349.1580 & 10474.7391 & 904764.6388 \\
\hline 52 & 1560 & 9,679 & 322.63 & 901160 & 0.0004 & 2.9901 & 345.0693 & 10352.0795 & 915116.7183 \\
\hline 53 & 1590 & 9,237 & 307.90 & 910397 & 0.0003 & 2.9543 & 341.0925 & 10232.7741 & 925349.4924 \\
\hline 54 & 1620 & 9,581 & 319.37 & 919978 & 0.0003 & 2.9195 & 337.2226 & 10116.6783 & 935466.1707 \\
\hline 55 & 1650 & 9,533 & 317.77 & 929511 & 0.0003 & 2.8856 & 333.4552 & 10003.6564 & 945469.8270 \\
\hline 56 & 1680 & 8,632 & 287.73 & 938143 & 0.0003 & 2.8526 & 329.7860 & 9893.5802 & 955363.4072 \\
\hline 57 & 1710 & 9,691 & 323.03 & 947834 & 0.0003 & 2.8205 & 326.2110 & 9786.3289 & 965149.7361 \\
\hline 58 & 1740 & 9,387 & 312.90 & 957221 & 0.0003 & 2.7891 & 322.7263 & 9681.7885 & 974831.5246 \\
\hline 59 & 1770 & 9,792 & 326.40 & 967013 & 0.0003 & 2.7586 & 319.3284 & 9579.8512 & 984411.3758 \\
\hline 60 & 1800 & 9,027 & 300.90 & 976040 & 0.0003 & 2.7287 & 316.0138 & 9480.4151 & 993891.7909 \\
\hline 61 & 1830 & 9,390 & 313.00 & 985430 & 0.0003 & 2.6996 & 312.7795 & 9383.3836 & 1003275.1745 \\
\hline 62 & 1860 & 9,374 & 312.47 & 994804 & 0.0003 & 2.6712 & 309.6222 & 9288.6654 & 1012563.8399 \\
\hline 63 & 1890 & 9,025 & 300.83 & 1003829 & 0.0003 & 2.6435 & 306.5391 & 9196.1737 & 1021760.0136 \\
\hline 64 & 1920 & 9,180 & 306.00 & 1013009 & 0.0003 & 2.6164 & 303.5275 & 9105.8262 & 1030865.8398 \\
\hline 65 & 1950 & 8,799 & 293.30 & 1021808 & 0.0003 & 2.5900 & 300.5848 & 9017.5449 & 1039883.3846 \\
\hline 66 & 1980 & 8,849 & 294.97 & 1030657 & 0.0003 & 2.5641 & 297.7085 & 8931.2554 & 1048814.6401 \\
\hline 67 & 2010 & 8,511 & 283.70 & 1039168 & 0.0003 & 2.5388 & 294.8962 & 8846.8873 & 1057661.5274 \\
\hline 68 & 2040 & 7,665 & 255.50 & 1046833 & 0.0002 & 2.5140 & 292.1458 & 8764.3732 & 1066425.9006 \\
\hline 69 & 2070 & 8,705 & 290.17 & 1055538 & 0.0003 & 2.4898 & 289.4550 & 8683.6492 & 1075109.5497 \\
\hline 70 & 2100 & 8,402 & 280.07 & 1063940 & 0.0003 & 2.4661 & 286.8218 & 8604.6542 & 1083714.2040 \\
\hline 71 & 2130 & 8,088 & 269.60 & 1072028 & 0.0003 & 2.4430 & 284.2443 & 8527.3302 & 1092241.5342 \\
\hline 72 & 2160 & 8,275 & 275.83 & 1080303 & 0.0003 & 2.4203 & 281.7207 & 8451.6216 & 1100693.1559 \\
\hline 73 & 2190 & 8,685 & 289.50 & 1088988 & 0.0003 & 2.3980 & 279.2492 & 8377.4755 & 1109070.6314 \\
\hline 74 & 2220 & 8,422 & 280.73 & 1097410 & 0.0003 & 2.3763 & 276.8280 & 8304.8412 & 1117375.4726 \\
\hline
\end{tabular}




\begin{tabular}{|c|c|c|c|c|c|c|c|c|c|}
\hline 75 & 2250 & 7,936 & 264.53 & 1105346 & 0.0002 & 2.3549 & 274.4557 & 8233.6704 & 1125609.1431 \\
\hline 76 & 2280 & 8,090 & 269.67 & 1113436 & 0.0002 & 2.3340 & 272.1306 & 8163.9168 & 1133773.0599 \\
\hline 77 & 2310 & 7,675 & 255.83 & 1121111 & 0.0002 & 2.3135 & 269.8512 & 8095.5361 & 1141868.5960 \\
\hline 78 & 2340 & 7,652 & 255.07 & 1128763 & 0.0002 & 2.2934 & 267.6162 & 8028.4859 & 1149897.0819 \\
\hline 79 & 2370 & 7,439 & 247.97 & 1136202 & 0.0002 & 2.2737 & 265.4242 & 7962.7257 & 1157859.8076 \\
\hline 80 & 2400 & 6,462 & 215.40 & 1142664 & 0.0002 & 2.2543 & 263.2739 & 7898.2164 & 1165758.0240 \\
\hline 81 & 2430 & 7,867 & 262.23 & 1150531 & 0.0002 & 2.2354 & 261.1640 & 7834.9208 & 1173592.9449 \\
\hline 82 & 2460 & 7,713 & 257.10 & 1158244 & 0.0002 & 2.2167 & 259.0934 & 7772.8032 & 1181365.7480 \\
\hline 83 & 2490 & 8,128 & 270.93 & 1166372 & 0.0002 & 2.1984 & 257.0610 & 7711.8290 & 1189077.5770 \\
\hline 84 & 2520 & 7,740 & 258.00 & 1174112 & 0.0002 & 2.1805 & 255.0655 & 7651.9653 & 1196729.5423 \\
\hline 85 & 2550 & 7,846 & 261.53 & 1181958 & 0.0002 & 2.1629 & 253.1060 & 7593.1805 & 1204322.7229 \\
\hline 86 & 2580 & 7,662 & 255.40 & 1189620 & 0.0002 & 2.1456 & 251.1815 & 7535.4440 & 1211858.1669 \\
\hline 87 & 2610 & 7,270 & 242.33 & 1196890 & 0.0002 & 2.1285 & 249.2909 & 7478.7266 & 1219336.8936 \\
\hline 88 & 2640 & 7,449 & 248.30 & 1204339 & 0.0002 & 2.1118 & 247.4333 & 7423.0001 & 1226759.8937 \\
\hline 89 & 2670 & 6,966 & 232.20 & 1211305 & 0.0002 & 2.0954 & 245.6079 & 7368.2374 & 1234128.1311 \\
\hline 90 & 2700 & 7,369 & 245.63 & 1218674 & 0.0002 & 2.0793 & 243.8137 & 7314.4123 & 1241442.5433 \\
\hline 91 & 2730 & 14,248 & 474.93 & 1232922 & 0.0004 & 2.0634 & 242.0500 & 7261.4997 & 1248704.0430 \\
\hline 92 & 2760 & 13,208 & 440.27 & 1246130 & 0.0004 & 2.0478 & 240.3158 & 7209.4754 & 1255913.5185 \\
\hline 93 & 2790 & 14,394 & 479.80 & 1260524 & 0.0004 & 2.0325 & 238.6105 & 7158.3161 & 1263071.8346 \\
\hline 94 & 2820 & 13,534 & 451.13 & 1274058 & 0.0004 & 2.0174 & 236.9333 & 7107.9992 & 1270179.8338 \\
\hline 95 & 2850 & 14,438 & 481.27 & 1288496 & 0.0004 & 2.0025 & 235.2834 & 7058.5030 & 1277238.3368 \\
\hline 96 & 2880 & 7,017 & 233.90 & 1295513 & 0.0002 & 1.9879 & 233.6602 & 7009.8066 & 1284248.1434 \\
\hline 97 & 2910 & 7,049 & 234.97 & 1302562 & 0.0002 & 1.9736 & 232.0630 & 6961.8897 & 1291210.0331 \\
\hline 98 & 2940 & 6,638 & 221.27 & 1309200 & 0.0002 & 1.9594 & 230.4911 & 6914.7329 & 1298124.7660 \\
\hline 99 & 2970 & 6,458 & 215.27 & 1315658 & 0.0002 & 1.9455 & 228.9439 & 6868.3172 & 1304993.0833 \\
\hline 100 & 3000 & 6,886 & 229.53 & 1322544 & 0.0002 & 1.9318 & 227.4208 & 6822.6245 & 1311815.7077 \\
\hline 101 & 3030 & 6,889 & 229.63 & 1329433 & 0.0002 & 1.9183 & 225.9212 & 6777.6371 & 1318593.3448 \\
\hline 102 & 3060 & 6,785 & 226.17 & 1336218 & 0.0002 & 1.9051 & 224.4446 & 6733.3380 & 1325326.6828 \\
\hline 103 & 3090 & 6,536 & 217.87 & 1342754 & 0.0002 & 1.8920 & 222.9904 & 6689.7107 & 1332016.3934 \\
\hline 104 & 3120 & 6,111 & 203.70 & 1348865 & 0.0002 & 1.8791 & 221.5580 & 6646.7393 & 1338663.1327 \\
\hline 105 & 3150 & 6,628 & 220.93 & 1355493 & 0.0002 & 1.8664 & 220.1469 & 6604.4084 & 1345267.5411 \\
\hline 106 & 3180 & 5,820 & 194.00 & 1361313 & 0.0001 & 1.8539 & 218.7568 & 6562.7030 & 1351830.2441 \\
\hline 107 & 3210 & 4,844 & 161.47 & 1366157 & 0.0001 & 1.8416 & 217.3870 & 6521.6088 & 1358351.8529 \\
\hline 108 & 3240 & 2,578 & 85.93 & 1368735 & 0.0001 & 1.8294 & 216.0371 & 6481.1117 & 1364832.9646 \\
\hline 109 & 3270 & 1,009 & 33.63 & 1369744 & 0.0000 & 1.8175 & 214.7066 & 6441.1981 & 1371274.1627 \\
\hline 110 & 3300 & 7,538 & 251.27 & 1377282 & 0.0002 & 1.8057 & 213.3952 & 6401.8550 & 1377676.0177 \\
\hline 111 & 3330 & 6,780 & 226.00 & 1384062 & 0.0002 & 1.7940 & 212.1023 & 6363.0695 & 1384039.0872 \\
\hline 112 & 3360 & 5,067 & 168.90 & 1389129 & 0.0001 & 1.7826 & 210.8276 & 6324.8294 & 1390363.9166 \\
\hline
\end{tabular}


Well No. 3 Calculations (Duong Model)

\begin{tabular}{|c|c|c|c|c|c|c|c|c|c|}
\hline \multicolumn{2}{|c|}{ Time } & \multirow{2}{*}{$\begin{array}{c}\text { Flow Rate } \\
\text { Mscf/Month }\end{array}$} & \multirow[b]{2}{*}{$\begin{array}{c}q \\
\text { (Mscf/d) }\end{array}$} & \multirow[b]{2}{*}{$\begin{array}{c}\text { Gp } \\
\text { (Mscf) }\end{array}$} & \multirow[b]{2}{*}{$\begin{array}{l}\text { (q/Gp) } \\
\text { (1/day) }\end{array}$} & \multirow[b]{2}{*}{$t(a, m)$} & \multirow[b]{2}{*}{$\begin{array}{l}\text { q-Duong } \\
\text { (Mscf/d) }\end{array}$} & \multirow[b]{2}{*}{$\begin{array}{l}\text { q-Duong } \\
\text { (Mscf/m) }\end{array}$} & \multirow[b]{2}{*}{$\begin{array}{c}\text { Gp_Doung } \\
\text { (Mscf) }\end{array}$} \\
\hline Month & Days & & & & & & & & \\
\hline 1 & 30 & 139490 & 4650 & 139490 & 0.0333 & 3.62 & 5163.02 & 154890.52 & 154890.52 \\
\hline 2 & 60 & 156342 & 5211 & 295832 & 0.0176 & 3.23 & 4615.54 & 138466.19 & 293356.72 \\
\hline 3 & 90 & 143525 & 4784 & 439357 & 0.0109 & 2.90 & 4141.83 & 124254.79 & 417611.50 \\
\hline 4 & 120 & 123918 & 4131 & 563275 & 0.0073 & 2.64 & 3769.42 & 113082.54 & 530694.05 \\
\hline 5 & 150 & 116830 & 3894 & 680105 & 0.0057 & 2.43 & 3471.28 & 104138.53 & 634832.58 \\
\hline 6 & 180 & 98102 & 3270 & 778207 & 0.0042 & 2.25 & 3226.68 & 96800.36 & 731632.94 \\
\hline 7 & 210 & 88543 & 2951 & 866750 & 0.0034 & 2.11 & 3021.64 & 90649.30 & 822282.23 \\
\hline 8 & 240 & 60454 & 2015 & 927204 & 0.0022 & 1.98 & 2846.70 & 85401.10 & 907683.33 \\
\hline 9 & 270 & 46030 & 1534 & 973234 & 0.0016 & 1.88 & 2695.24 & 80857.24 & 988540.56 \\
\hline 10 & 300 & 58339 & 1945 & 1031573 & 0.0019 & 1.78 & 2562.50 & 76874.93 & 1065415.50 \\
\hline 11 & 330 & 52154 & 1738 & 1083727 & 0.0016 & 1.70 & 2444.96 & 73348.71 & 1138764.21 \\
\hline 12 & 360 & 48045 & 1602 & 1131772 & 0.0014 & 1.63 & 2339.96 & 70198.82 & 1208963.02 \\
\hline 13 & 390 & 41601 & 1387 & 1173373 & 0.0012 & 1.56 & 2245.46 & 67363.70 & 1276326.72 \\
\hline 14 & 420 & 41623 & 1387 & 1214996 & 0.0011 & 1.50 & 2159.83 & 64795.01 & 1341121.73 \\
\hline 15 & 450 & 62059 & 2069 & 1277055 & 0.0016 & 1.44 & 2081.81 & 62454.18 & 1403575.91 \\
\hline 16 & 480 & 59340 & 1978 & 1336395 & 0.0015 & 1.39 & 2010.33 & 60310.00 & 1463885.91 \\
\hline 17 & 510 & 46266 & 1542 & 1382661 & 0.0011 & 1.35 & 1944.56 & 58336.93 & 1522222.84 \\
\hline 18 & 540 & 49801 & 1660 & 1432462 & 0.0012 & 1.30 & 1883.79 & 56513.84 & 1578736.68 \\
\hline 19 & 570 & 36184 & 1206 & 1468646 & 0.0008 & 1.26 & 1827.43 & 54823.04 & 1633559.72 \\
\hline 20 & 600 & 38899 & 1297 & 1507545 & 0.0009 & 1.23 & 1774.99 & 53249.66 & 1686809.38 \\
\hline 21 & 630 & 59044 & 1968 & 1566589 & 0.0013 & 1.19 & 1726.03 & 51781.02 & 1738590.40 \\
\hline 22 & 660 & 27573 & 919 & 1594162 & 0.0006 & 1.16 & 1680.21 & 50406.29 & 1788996.69 \\
\hline 23 & 690 & 49524 & 1651 & 1643686 & 0.0010 & 1.13 & 1637.20 & 49116.14 & 1838112.83 \\
\hline 24 & 720 & 62830 & 2094 & 1706516 & 0.0012 & 1.10 & 1596.75 & 47902.48 & 1886015.31 \\
\hline 25 & 750 & 66977 & 2233 & 1773493 & 0.0013 & 1.07 & 1558.61 & 46758.24 & 1932773.55 \\
\hline 26 & 780 & 57594 & 1920 & 1831087 & 0.0010 & 1.05 & 1522.57 & 45677.24 & 1978450.79 \\
\hline 27 & 810 & 57390 & 1913 & 1888477 & 0.0010 & 1.02 & 1488.47 & 44654.05 & 2023104.84 \\
\hline 28 & 840 & 49702 & 1657 & 1938179 & 0.0009 & 1.00 & 1456.13 & 43683.84 & 2066788.69 \\
\hline 29 & 870 & 48909 & 1630 & 1987088 & 0.0008 & 0.98 & 1425.41 & 42762.35 & 2109551.04 \\
\hline 30 & 900 & 49114 & 1637 & 2036202 & 0.0008 & 0.96 & 1396.19 & 41885.76 & 2151436.80 \\
\hline 31 & 930 & 44564 & 1485 & 2080766 & 0.0007 & 0.94 & 1368.36 & 41050.66 & 2192487.46 \\
\hline 32 & 960 & 44412 & 1480 & 2125178 & 0.0007 & 0.92 & 1341.80 & 40253.99 & 2232741.45 \\
\hline 33 & 990 & 51766 & 1726 & 2176944 & 0.0008 & 0.90 & 1316.43 & 39492.99 & 2272234.44 \\
\hline
\end{tabular}




\begin{tabular}{|c|c|c|c|c|c|c|c|c|c|}
\hline 34 & 1020 & 49955 & 1665 & 2226899 & 0.0007 & 0.88 & 1292.17 & 38765.18 & 2310999.62 \\
\hline 35 & 1050 & 47995 & 1600 & 2274894 & 0.0007 & 0.87 & 1268.94 & 38068.30 & 2349067.92 \\
\hline 36 & 1080 & 43215 & 1441 & 2318109 & 0.0006 & 0.85 & 1246.68 & 37400.29 & 2386468.21 \\
\hline 37 & 1110 & 42290 & 1410 & 2360399 & 0.0006 & 0.84 & 1225.31 & 36759.30 & 2423227.51 \\
\hline 38 & 1140 & 38981 & 1299 & 2399380 & 0.0005 & 0.82 & 1204.79 & 36143.61 & 2459371.12 \\
\hline 39 & 1170 & 38422 & 1281 & 2437802 & 0.0005 & 0.81 & 1185.06 & 35551.68 & 2494922.80 \\
\hline 40 & 1200 & 38034 & 1268 & 2475836 & 0.0005 & 0.80 & 1166.07 & 34982.07 & 2529904.88 \\
\hline 41 & 1230 & 32816 & 1094 & 2508652 & 0.0004 & 0.78 & 1147.78 & 34433.47 & 2564338.35 \\
\hline 42 & 1260 & 35300 & 1177 & 2543952 & 0.0005 & 0.77 & 1130.16 & 33904.68 & 2598243.03 \\
\hline 43 & 1290 & 34784 & 1159 & 2578736 & 0.0004 & 0.76 & 1113.15 & 33394.57 & 2631637.60 \\
\hline 44 & 1320 & 34740 & 1158 & 2613476 & 0.0004 & 0.75 & 1096.74 & 32902.12 & 2664539.72 \\
\hline 45 & 1350 & 33608 & 1120 & 2647084 & 0.0004 & 0.74 & 1080.88 & 32426.37 & 2696966.09 \\
\hline 46 & 1380 & 29345 & 978 & 2676429 & 0.0004 & 0.72 & 1065.55 & 31966.45 & 2728932.54 \\
\hline 47 & 1410 & 30133 & 1004 & 2706562 & 0.0004 & 0.71 & 1050.72 & 31521.53 & 2760454.07 \\
\hline 48 & 1440 & 29324 & 977 & 2735886 & 0.0004 & 0.70 & 1036.36 & 31090.85 & 2791544.92 \\
\hline 49 & 1470 & 30641 & 1021 & 2766527 & 0.0004 & 0.69 & 1022.46 & 30673.69 & 2822218.61 \\
\hline 50 & 1500 & 28732 & 958 & 2795259 & 0.0003 & 0.68 & 1008.98 & 30269.41 & 2852488.01 \\
\hline 51 & 1530 & 28764 & 959 & 2824023 & 0.0003 & 0.68 & 995.91 & 29877.37 & 2882365.38 \\
\hline 52 & 1560 & 27657 & 922 & 2851680 & 0.0003 & 0.67 & 983.23 & 29497.00 & 2911862.38 \\
\hline 53 & 1590 & 27322 & 911 & 2879002 & 0.0003 & 0.66 & 970.93 & 29127.76 & 2940990.13 \\
\hline 54 & 1620 & 25858 & 862 & 2904860 & 0.0003 & 0.65 & 958.97 & 28769.14 & 2969759.27 \\
\hline 55 & 1650 & 25081 & 836 & 2929941 & 0.0003 & 0.64 & 947.36 & 28420.66 & 2998179.93 \\
\hline 56 & 1680 & 25429 & 848 & 2955370 & 0.0003 & 0.63 & 936.06 & 28081.89 & 3026261.82 \\
\hline 57 & 1710 & 25652 & 855 & 2981022 & 0.0003 & 0.63 & 925.08 & 27752.39 & 3054014.22 \\
\hline 58 & 1740 & 23435 & 781 & 3004457 & 0.0003 & 0.62 & 914.39 & 27431.78 & 3081445.99 \\
\hline 59 & 1770 & 26241 & 875 & 3030698 & 0.0003 & 0.61 & 903.99 & 27119.67 & 3108565.67 \\
\hline 60 & 1800 & 25197 & 840 & 3055895 & 0.0003 & 0.60 & 893.86 & 26815.72 & 3135381.39 \\
\hline 61 & 1830 & 23809 & 794 & 3079704 & 0.0003 & 0.60 & 883.99 & 26519.60 & 3161900.99 \\
\hline 62 & 1860 & 17956 & 599 & 3097660 & 0.0002 & 0.59 & 874.37 & 26230.99 & 3188131.98 \\
\hline 63 & 1890 & 24069 & 802 & 3121729 & 0.0003 & 0.58 & 864.99 & 25949.60 & 3214081.58 \\
\hline 64 & 1920 & 23127 & 771 & 3144856 & 0.0002 & 0.58 & 855.84 & 25675.14 & 3239756.72 \\
\hline 65 & 1950 & 20766 & 692 & 3165622 & 0.0002 & 0.57 & 846.91 & 25407.35 & 3265164.06 \\
\hline 66 & 1980 & 23203 & 773 & 3188825 & 0.0002 & 0.56 & 838.20 & 25145.97 & 3290310.04 \\
\hline 67 & 2010 & 21304 & 710 & 3210129 & 0.0002 & 0.56 & 829.69 & 24890.77 & 3315200.81 \\
\hline 68 & 2040 & 23006 & 767 & 3233135 & 0.0002 & 0.55 & 821.38 & 24641.53 & 3339842.33 \\
\hline 69 & 2070 & 25986 & 866 & 3259121 & 0.0003 & 0.55 & 813.27 & 24398.01 & 3364240.35 \\
\hline 70 & 2100 & 19497 & 650 & 3278618 & 0.0002 & 0.54 & 805.33 & 24160.03 & 3388400.37 \\
\hline 71 & 2130 & 20433 & 681 & 3299051 & 0.0002 & 0.54 & 797.58 & 23927.38 & 3412327.75 \\
\hline 72 & 2160 & 18257 & 609 & 3317308 & 0.0002 & 0.53 & 790.00 & 23699.87 & 3436027.62 \\
\hline 73 & 2190 & 16958 & 565 & 3334266 & 0.0002 & 0.52 & 782.58 & 23477.34 & 3459504.97 \\
\hline
\end{tabular}




\begin{tabular}{|c|c|c|c|c|c|c|c|c|c|}
\hline 74 & 2220 & 19084 & 636 & 3353350 & 0.0002 & 0.52 & 775.32 & 23259.61 & 3482764.58 \\
\hline 75 & 2250 & 21110 & 704 & 3374460 & 0.0002 & 0.51 & 768.22 & 23046.52 & 3505811.10 \\
\hline 76 & 2280 & 20551 & 685 & 3395011 & 0.0002 & 0.51 & 761.26 & 22837.92 & 3528649.02 \\
\hline 77 & 2310 & 18159 & 605 & 3413170 & 0.0002 & 0.50 & 754.46 & 22633.65 & 3551282.67 \\
\hline 78 & 2340 & 20413 & 680 & 3433583 & 0.0002 & 0.50 & 747.79 & 22433.59 & 3573716.26 \\
\hline 79 & 2370 & 18643 & 621 & 3452226 & 0.0002 & 0.50 & 741.25 & 22237.59 & 3595953.85 \\
\hline 80 & 2400 & 17841 & 595 & 3470067 & 0.0002 & 0.49 & 734.85 & 22045.53 & 3617999.39 \\
\hline 81 & 2430 & 17827 & 594 & 3487894 & 0.0002 & 0.49 & 728.58 & 21857.28 & 3639856.67 \\
\hline 82 & 2460 & 16309 & 544 & 3504203 & 0.0002 & 0.48 & 722.42 & 21672.73 & 3661529.40 \\
\hline 83 & 2490 & 20280 & 676 & 3524483 & 0.0002 & 0.48 & 716.39 & 21491.77 & 3683021.17 \\
\hline 84 & 2520 & 21320 & 711 & 3545803 & 0.0002 & 0.47 & 710.48 & 21314.28 & 3704335.45 \\
\hline 85 & 2550 & 18637 & 621 & 3564440 & 0.0002 & 0.47 & 704.67 & 21140.16 & 3725475.60 \\
\hline 86 & 2580 & 17431 & 581 & 3581871 & 0.0002 & 0.47 & 698.98 & 20969.31 & 3746444.91 \\
\hline 87 & 2610 & 15456 & 515 & 3597327 & 0.0001 & 0.46 & 693.39 & 20801.64 & 3767246.55 \\
\hline 88 & 2640 & 14987 & 500 & 3612314 & 0.0001 & 0.46 & 687.90 & 20637.05 & 3787883.61 \\
\hline 89 & 2670 & 14257 & 475 & 3626571 & 0.0001 & 0.45 & 682.52 & 20475.46 & 3808359.07 \\
\hline 90 & 2700 & 13557 & 452 & 3640128 & 0.0001 & 0.45 & 677.23 & 20316.79 & 3828675.86 \\
\hline 91 & 2730 & 13851 & 462 & 3653979 & 0.0001 & 0.45 & 672.03 & 20160.94 & 3848836.80 \\
\hline 92 & 2760 & 16898 & 563 & 3670877 & 0.0002 & 0.44 & 666.93 & 20007.85 & 3868844.65 \\
\hline 93 & 2790 & 15711 & 524 & 3686588 & 0.0001 & 0.44 & 661.91 & 19857.43 & 3888702.09 \\
\hline 94 & 2820 & 17055 & 569 & 3703643 & 0.0002 & 0.44 & 656.99 & 19709.62 & 3908411.71 \\
\hline 95 & 2850 & 13931 & 464 & 3717574 & 0.0001 & 0.43 & 652.14 & 19564.34 & 3927976.05 \\
\hline 96 & 2880 & 14271 & 476 & 3731845 & 0.0001 & 0.43 & 647.38 & 19421.53 & 3947397.58 \\
\hline 97 & 2910 & 13153 & 438 & 3744998 & 0.0001 & 0.43 & 642.70 & 19281.12 & 3966678.71 \\
\hline 98 & 2940 & 11133 & 371 & 3756131 & 0.0001 & 0.42 & 638.10 & 19143.05 & 3985821.76 \\
\hline 99 & 2970 & 14595 & 487 & 3770726 & 0.0001 & 0.42 & 633.58 & 19007.26 & 4004829.02 \\
\hline 100 & 3000 & 13221 & 441 & 3783947 & 0.0001 & 0.42 & 629.12 & 18873.69 & 4023702.71 \\
\hline 101 & 3030 & 12720 & 424 & 3796667 & 0.0001 & 0.41 & 624.74 & 18742.28 & 4042444.99 \\
\hline 102 & 3060 & 15617 & 521 & 3812284 & 0.0001 & 0.41 & 620.43 & 18612.98 & 4061057.97 \\
\hline 103 & 3090 & 15921 & 531 & 3828205 & 0.0001 & 0.41 & 616.19 & 18485.74 & 4079543.72 \\
\hline 104 & 3120 & 14757 & 492 & 3842962 & 0.0001 & 0.40 & 612.02 & 18360.51 & 4097904.23 \\
\hline 105 & 3150 & 13796 & 460 & 3856758 & 0.0001 & 0.40 & 607.91 & 18237.23 & 4116141.46 \\
\hline 106 & 3180 & 13553 & 452 & 3870311 & 0.0001 & 0.40 & 603.86 & 18115.87 & 4134257.33 \\
\hline 107 & 3210 & 13822 & 461 & 3884133 & 0.0001 & 0.40 & 599.88 & 17996.36 & 4152253.69 \\
\hline 108 & 3240 & 14266 & 476 & 3898399 & 0.0001 & 0.39 & 595.96 & 17878.68 & 4170132.37 \\
\hline 109 & 3270 & 14104 & 470 & 3912503 & 0.0001 & 0.39 & 592.09 & 17762.77 & 4187895.14 \\
\hline 110 & 3300 & 15514 & 517 & 3928017 & 0.0001 & 0.39 & 588.29 & 17648.60 & 4205543.74 \\
\hline 111 & 3330 & 16713 & 557 & 3944730 & 0.0001 & 0.38 & 584.54 & 17536.12 & 4223079.86 \\
\hline 112 & 3360 & 16966 & 566 & 3961696 & 0.0001 & 0.38 & 580.84 & 17425.30 & 4240505.16 \\
\hline 113 & 3390 & 17365 & 579 & 3979061 & 0.0001 & 0.38 & 577.20 & 17316.10 & 4257821.26 \\
\hline
\end{tabular}




\begin{tabular}{|l|l|l|l|l|l|l|l|l|l|}
114 & 3420 & 15965 & 532 & 3995026 & 0.0001 & 0.38 & 573.62 & 17208.48 & 4275029.73 \\
\hline 115 & 3450 & 16133 & 538 & 4011159 & 0.0001 & 0.37 & 570.08 & 17102.40 & 4292132.13 \\
\hline 116 & 3450 & 16133 & 538 & 4027292 & 0.0001 & 0.37 & 570.08 & 17102.40 & 4309234.53 \\
\hline 117 & 3450 & 16133 & 538 & 4043425 & 0.0001 & 0.37 & 570.08 & 17102.40 & 4326336.93 \\
\hline 118 & 3450 & 16133 & 538 & 4059558 & 0.0001 & 0.37 & 570.08 & 17102.40 & 4343439.33 \\
\hline 119 & 3450 & 16133 & 538 & 4075691 & 0.0001 & 0.37 & 570.08 & 17102.40 & 4360541.73 \\
\hline 120 & 3450 & 16133 & 538 & 4091824 & 0.0001 & 0.37 & 570.08 & 17102.40 & 4377644.13 \\
\hline 121 & 3450 & 16133 & 538 & 4107957 & 0.0001 & 0.37 & 570.08 & 17102.40 & 4394746.53 \\
\hline 122 & 3450 & 16133 & 538 & 4124090 & 0.0001 & 0.37 & 570.08 & 17102.40 & 4411848.92 \\
\hline 123 & 3450 & 16133 & 538 & 4140223 & 0.0001 & 0.37 & 570.08 & 17102.40 & 4428951.32 \\
\hline
\end{tabular}


Well No. 4H Calculations (Duong Model)

\begin{tabular}{|c|c|c|c|c|c|c|c|c|c|}
\hline \multicolumn{2}{|c|}{ Time } & \multirow{2}{*}{$\begin{array}{c}\text { Flow Rate } \\
\text { Mscf/Month }\end{array}$} & \multirow[b]{2}{*}{$\begin{array}{c}q \\
(\mathrm{Mscf} / \mathrm{d})\end{array}$} & \multirow[b]{2}{*}{$\begin{array}{c}\text { Gp } \\
\text { (Mscf) }\end{array}$} & \multirow[b]{2}{*}{$\begin{array}{l}\text { (q/Gp) } \\
\text { (1/day) }\end{array}$} & \multirow[b]{2}{*}{$t(a, m)$} & \multirow[b]{2}{*}{$\begin{array}{l}\text { q-Duong } \\
\text { (Mscf/d) }\end{array}$} & \multirow[b]{2}{*}{$\begin{array}{l}\text { q-Duong } \\
\text { (Mscf/m) }\end{array}$} & \multirow[b]{2}{*}{$\begin{array}{c}\text { Gp_Doung } \\
\text { (Mscf) }\end{array}$} \\
\hline Month & Days & & & & & & & & \\
\hline 1 & 30 & 37,032 & 1234.40 & 37032 & 0.0333 & 13.8020 & 2437.90 & 73136.92 & 73136.92 \\
\hline 2 & 60 & 82,849 & 2761.63 & 119881 & 0.0230 & 14.3221 & 2528.03 & 75840.84 & 148977.76 \\
\hline 3 & 90 & 77,704 & 2590.13 & 197585 & 0.0131 & 13.8373 & 2444.02 & 73320.51 & 222298.27 \\
\hline 4 & 120 & 76,042 & 2534.73 & 273627 & 0.0093 & 13.1994 & 2333.47 & 70004.20 & 292302.47 \\
\hline 5 & 150 & 71,697 & 2389.90 & 345324 & 0.0069 & 12.5706 & 2224.51 & 66735.23 & 359037.70 \\
\hline 6 & 180 & 68,230 & 2274.33 & 413554 & 0.0055 & 11.9884 & 2123.62 & 63708.65 & 422746.36 \\
\hline 7 & 210 & 65,162 & 2172.07 & 478716 & 0.0045 & 11.4588 & 2031.85 & 60955.62 & 483701.98 \\
\hline 8 & 240 & 61,609 & 2053.63 & 540325 & 0.0038 & 10.9789 & 1948.70 & 58460.94 & 542162.92 \\
\hline 9 & 270 & 64,212 & 2140.40 & 604537 & 0.0035 & 10.5436 & 1873.25 & 56197.52 & 598360.44 \\
\hline 10 & 300 & 57,347 & 1911.57 & 661884 & 0.0029 & 10.1473 & 1804.58 & 54137.32 & 652497.75 \\
\hline 11 & 330 & 56,201 & 1873.37 & 718085 & 0.0026 & 9.7852 & 1741.83 & 52254.78 & 704752.53 \\
\hline 12 & 360 & 52,352 & 1745.07 & 770437 & 0.0023 & 9.4529 & 1684.25 & 50527.63 & 755280.16 \\
\hline 13 & 390 & 51,359 & 1711.97 & 821796 & 0.0021 & 9.1469 & 1631.23 & 48936.79 & 804216.95 \\
\hline 14 & 420 & 49,412 & 1647.07 & 871208 & 0.0019 & 8.8640 & 1582.20 & 47466.03 & 851682.97 \\
\hline 15 & 450 & 43,644 & 1454.80 & 914852 & 0.0016 & 8.6015 & 1536.72 & 46101.51 & 897784.48 \\
\hline 16 & 480 & 45,445 & 1514.83 & 960297 & 0.0016 & 8.3572 & 1494.38 & 44831.45 & 942615.93 \\
\hline 17 & 510 & 43,723 & 1457.43 & 1004020 & 0.0015 & 8.1292 & 1454.86 & 43645.74 & 986261.67 \\
\hline 18 & 540 & 43,848 & 1461.60 & 1047868 & 0.0014 & 7.9156 & 1417.86 & 42535.68 & 1028797.35 \\
\hline 19 & 570 & 41,271 & 1375.70 & 1089139 & 0.0013 & 7.7152 & 1383.13 & 41493.75 & 1070291.10 \\
\hline 20 & 600 & 41,481 & 1382.70 & 1130620 & 0.0012 & 7.5266 & 1350.45 & 40513.43 & 1110804.54 \\
\hline 21 & 630 & 40,164 & 1338.80 & 1170784 & 0.0011 & 7.3488 & 1319.63 & 39589.01 & 1150393.55 \\
\hline 22 & 660 & 38,148 & 1271.60 & 1208932 & 0.0011 & 7.1808 & 1290.52 & 38715.50 & 1189109.05 \\
\hline 23 & 690 & 38,605 & 1286.83 & 1247537 & 0.0010 & 7.0217 & 1262.95 & 37888.49 & 1226997.54 \\
\hline 24 & 720 & 36,131 & 1204.37 & 1283668 & 0.0009 & 6.8708 & 1236.80 & 37104.10 & 1264101.64 \\
\hline 25 & 750 & 36,390 & 1213.00 & 1320058 & 0.0009 & 6.7275 & 1211.96 & 36358.86 & 1300460.50 \\
\hline 26 & 780 & 32,047 & 1068.23 & 1352105 & 0.0008 & 6.5911 & 1188.32 & 35649.71 & 1336110.21 \\
\hline 27 & 810 & 31,288 & 1042.93 & 1383393 & 0.0008 & 6.4611 & 1165.80 & 34973.88 & 1371084.09 \\
\hline 28 & 840 & 34,027 & 1134.23 & 1417420 & 0.0008 & 6.3370 & 1144.30 & 34328.91 & 1405413.00 \\
\hline 29 & 870 & 32,340 & 1078.00 & 1449760 & 0.0007 & 6.2185 & 1123.75 & 33712.58 & 1439125.58 \\
\hline 30 & 900 & 32,546 & 1084.87 & 1482306 & 0.0007 & 6.1050 & 1104.10 & 33122.88 & 1472248.46 \\
\hline 31 & 930 & 30,729 & 1024.30 & 1513035 & 0.0007 & 5.9964 & 1085.27 & 32558.00 & 1504806.46 \\
\hline 32 & 960 & 31,050 & 1035.00 & 1544085 & 0.0007 & 5.8922 & 1067.21 & 32016.28 & 1536822.74 \\
\hline 33 & 990 & 30,303 & 1010.10 & 1574388 & 0.0006 & 5.7921 & 1049.87 & 31496.24 & 1568318.98 \\
\hline
\end{tabular}




\begin{tabular}{|c|c|c|c|c|c|c|c|c|c|}
\hline 34 & 1020 & 28,372 & 945.73 & 1602760 & 0.0006 & 5.6960 & 1033.22 & 30996.49 & 1599315.47 \\
\hline 35 & 1050 & 29,436 & 981.20 & 1632196 & 0.0006 & 5.6035 & 1017.19 & 30515.79 & 1629831.25 \\
\hline 36 & 1080 & 21,991 & 733.03 & 1654187 & 0.0004 & 5.5145 & 1001.77 & 30052.99 & 1659884.24 \\
\hline 37 & 1110 & 29,717 & 990.57 & 1683904 & 0.0006 & 5.4287 & 986.90 & 29607.04 & 1689491.27 \\
\hline 38 & 1140 & 26,858 & 895.27 & 1710762 & 0.0005 & 5.3460 & 972.57 & 29176.96 & 1718668.24 \\
\hline 39 & 1170 & 22,476 & 749.20 & 1733238 & 0.0004 & 5.2662 & 958.73 & 28761.88 & 1747430.12 \\
\hline 40 & 1200 & 25,492 & 849.73 & 1758730 & 0.0005 & 5.1890 & 945.37 & 28360.96 & 1775791.08 \\
\hline 41 & 1230 & 22,674 & 755.80 & 1781404 & 0.0004 & 5.1145 & 932.45 & 27973.44 & 1803764.52 \\
\hline 42 & 1260 & 21,505 & 716.83 & 1802909 & 0.0004 & 5.0424 & 919.95 & 27598.61 & 1831363.13 \\
\hline 43 & 1290 & 24,698 & 823.27 & 1827607 & 0.0005 & 4.9726 & 907.86 & 27235.82 & 1858598.94 \\
\hline 44 & 1320 & 24,466 & 815.53 & 1852073 & 0.0004 & 4.9050 & 896.15 & 26884.45 & 1885483.39 \\
\hline 45 & 1350 & 22,864 & 762.13 & 1874937 & 0.0004 & 4.8395 & 884.80 & 26543.94 & 1912027.33 \\
\hline 46 & 1380 & 22,315 & 743.83 & 1897252 & 0.0004 & 4.7760 & 873.79 & 26213.76 & 1938241.08 \\
\hline 47 & 1410 & 19,978 & 665.93 & 1917230 & 0.0003 & 4.7144 & 863.11 & 25893.41 & 1964134.49 \\
\hline 48 & 1440 & 24,971 & 832.37 & 1942201 & 0.0004 & 4.6546 & 852.75 & 25582.43 & 1989716.92 \\
\hline 49 & 1470 & 9,322 & 310.73 & 1951523 & 0.0002 & 4.5965 & 842.68 & 25280.40 & 2014997.32 \\
\hline 50 & 1500 & 23,565 & 785.50 & 1975088 & 0.0004 & 4.5400 & 832.90 & 24986.89 & 2039984.21 \\
\hline 51 & 1530 & 48,351 & 1611.70 & 2023439 & 0.0008 & 4.4851 & 823.38 & 24701.54 & 2064685.75 \\
\hline 52 & 1560 & 37,557 & 1251.90 & 2060996 & 0.0006 & 4.4317 & 814.13 & 24423.99 & 2089109.74 \\
\hline 53 & 1590 & 31,858 & 1061.93 & 2092854 & 0.0005 & 4.3798 & 805.13 & 24153.89 & 2113263.63 \\
\hline 54 & 1620 & 26,764 & 892.13 & 2119618 & 0.0004 & 4.3292 & 796.36 & 23890.94 & 2137154.58 \\
\hline 55 & 1650 & 25,562 & 852.07 & 2145180 & 0.0004 & 4.2799 & 787.83 & 23634.84 & 2160789.41 \\
\hline 56 & 1680 & 25,062 & 835.40 & 2170242 & 0.0004 & 4.2319 & 779.51 & 23385.30 & 2184174.71 \\
\hline 57 & 1710 & 14,130 & 471.00 & 2184372 & 0.0002 & 4.1852 & 771.40 & 23142.06 & 2207316.78 \\
\hline 58 & 1740 & 44,225 & 1474.17 & 2228597 & 0.0007 & 4.1395 & 763.50 & 22904.87 & 2230221.65 \\
\hline 59 & 1770 & 32,789 & 1092.97 & 2261386 & 0.0005 & 4.0950 & 755.78 & 22673.49 & 2252895.14 \\
\hline 60 & 1800 & 25,795 & 859.83 & 2287181 & 0.0004 & 4.0516 & 748.26 & 22447.70 & 2275342.84 \\
\hline 61 & 1830 & 23,834 & 794.47 & 2311015 & 0.0003 & 4.0092 & 740.91 & 22227.29 & 2297570.13 \\
\hline 62 & 1860 & 20,447 & 681.57 & 2331462 & 0.0003 & 3.9678 & 733.73 & 22012.05 & 2319582.18 \\
\hline 63 & 1890 & 22,173 & 739.10 & 2353635 & 0.0003 & 3.9273 & 726.73 & 21801.79 & 2341383.96 \\
\hline 64 & 1920 & 18,936 & 631.20 & 2372571 & 0.0003 & 3.8878 & 719.88 & 21596.33 & 2362980.29 \\
\hline 65 & 1950 & 19,175 & 639.17 & 2391746 & 0.0003 & 3.8492 & 713.18 & 21395.50 & 2384375.79 \\
\hline 66 & 1980 & 18,126 & 604.20 & 2409872 & 0.0003 & 3.8114 & 706.64 & 21199.13 & 2405574.93 \\
\hline 67 & 2010 & 20,781 & 692.70 & 2430653 & 0.0003 & 3.7745 & 700.24 & 21007.08 & 2426582.00 \\
\hline 68 & 2040 & 20,785 & 692.83 & 2451438 & 0.0003 & 3.7383 & 693.97 & 20819.18 & 2447401.18 \\
\hline 69 & 2070 & 16,646 & 554.87 & 2468084 & 0.0002 & 3.7030 & 687.84 & 20635.30 & 2468036.48 \\
\hline
\end{tabular}


Well No. 6H Calculations (Duong Model)

\begin{tabular}{|c|c|c|c|c|c|c|c|c|c|}
\hline \multicolumn{2}{|c|}{ Time } & \multirow{2}{*}{$\begin{array}{c}\text { Flow Rate } \\
\text { Mscf/Month }\end{array}$} & \multirow[b]{2}{*}{$\begin{array}{c}q \\
\text { (Mscf/d) }\end{array}$} & \multirow[b]{2}{*}{$\begin{array}{c}\text { Gp } \\
\text { (Mscf) }\end{array}$} & \multirow[b]{2}{*}{$\begin{array}{l}\text { (q/Gp) } \\
\text { (1/day) }\end{array}$} & \multirow[b]{2}{*}{$t(a, m)$} & \multirow[b]{2}{*}{$\begin{array}{l}\text { q-Duong } \\
\text { (Mscf/d) }\end{array}$} & \multirow[b]{2}{*}{$\begin{array}{l}\text { q-Duong } \\
\text { (Mscf/m) }\end{array}$} & \multirow[b]{2}{*}{$\begin{array}{c}\text { Gp_Doung } \\
\text { (Mscf) }\end{array}$} \\
\hline Month & Days & & & & & & & & \\
\hline 1 & 30 & 22,174 & 739.13 & 22174 & 0.0333 & 5.3750 & 1476.23 & 44286.79 & 44286.79 \\
\hline 2 & 60 & 44,127 & 1470.90 & 66301 & 0.0222 & 5.2323 & 1437.19 & 43115.80 & 87402.59 \\
\hline 3 & 90 & 42,766 & 1425.53 & 109067 & 0.0131 & 4.9302 & 1354.52 & 40635.52 & 128038.11 \\
\hline 4 & 120 & 42,256 & 1408.53 & 151323 & 0.0093 & 4.6422 & 1275.68 & 38270.53 & 166308.64 \\
\hline 5 & 150 & 39,435 & 1314.50 & 190758 & 0.0069 & 4.3874 & 1205.97 & 36179.06 & 202487.70 \\
\hline 6 & 180 & 34,535 & 1151.17 & 225293 & 0.0051 & 4.1643 & 1144.93 & 34347.87 & 236835.56 \\
\hline 7 & 210 & 28,721 & 957.37 & 254014 & 0.0038 & 3.9683 & 1091.27 & 32738.21 & 269573.78 \\
\hline 8 & 240 & 28,523 & 950.77 & 282537 & 0.0034 & 3.7947 & 1043.76 & 31312.89 & 300886.67 \\
\hline 9 & 270 & 34,379 & 1145.97 & 316916 & 0.0036 & 3.6397 & 1001.36 & 30040.92 & 330927.58 \\
\hline 10 & 300 & 28,047 & 934.90 & 344963 & 0.0027 & 3.5005 & 963.25 & 28897.39 & 359824.97 \\
\hline 11 & 330 & 23,798 & 793.27 & 368761 & 0.0022 & 3.3744 & 928.75 & 27862.39 & 387687.35 \\
\hline 12 & 360 & 26,677 & 889.23 & 395438 & 0.0022 & 3.2596 & 897.33 & 26919.93 & 414607.29 \\
\hline 13 & 390 & 29,117 & 970.57 & 424555 & 0.0023 & 3.1545 & 868.57 & 26057.09 & 440664.38 \\
\hline 14 & 420 & 24,991 & 833.03 & 449546 & 0.0019 & 3.0578 & 842.11 & 25263.29 & 465927.67 \\
\hline 15 & 450 & 23,080 & 769.33 & 472626 & 0.0016 & 2.9685 & 817.66 & 24529.80 & 490457.47 \\
\hline 16 & 480 & 24,152 & 805.07 & 496778 & 0.0016 & 2.8856 & 794.98 & 23849.38 & 514306.85 \\
\hline 17 & 510 & 22,149 & 738.30 & 518927 & 0.0014 & 2.8084 & 773.86 & 23215.92 & 537522.77 \\
\hline 18 & 540 & 22,123 & 737.43 & 541050 & 0.0014 & 2.7364 & 754.14 & 22624.28 & 560147.05 \\
\hline 19 & 570 & 20,913 & 697.10 & 561963 & 0.0012 & 2.6688 & 735.67 & 22070.05 & 582217.10 \\
\hline 20 & 600 & 19,196 & 639.87 & 581159 & 0.0011 & 2.6054 & 718.32 & 21549.47 & 603766.57 \\
\hline 21 & 630 & 18,394 & 613.13 & 599553 & 0.0010 & 2.5457 & 701.98 & 21059.27 & 624825.84 \\
\hline 22 & 660 & 19,508 & 650.27 & 619061 & 0.0011 & 2.4894 & 686.55 & 20596.62 & 645422.46 \\
\hline 23 & 690 & 19,220 & 640.67 & 638281 & 0.0010 & 2.4361 & 671.97 & 20159.04 & 665581.50 \\
\hline 24 & 720 & 17,947 & 598.23 & 656228 & 0.0009 & 2.3856 & 658.15 & 19744.37 & 685325.87 \\
\hline 25 & 750 & 17,785 & 592.83 & 674013 & 0.0009 & 2.3376 & 645.02 & 19350.67 & 704676.54 \\
\hline 26 & 780 & 14,647 & 488.23 & 688660 & 0.0007 & 2.2920 & 632.54 & 18976.26 & 723652.80 \\
\hline 27 & 810 & 15,460 & 515.33 & 704120 & 0.0007 & 2.2486 & 620.65 & 18619.62 & 742272.43 \\
\hline 28 & 840 & 17,141 & 571.37 & 721261 & 0.0008 & 2.2071 & 609.31 & 18279.41 & 760551.83 \\
\hline 29 & 870 & 15,846 & 528.20 & 737107 & 0.0007 & 2.1675 & 598.48 & 17954.40 & 778506.23 \\
\hline 30 & 900 & 15,310 & 510.33 & 752417 & 0.0007 & 2.1297 & 588.12 & 17643.50 & 796149.73 \\
\hline 31 & 930 & 14,422 & 480.73 & 766839 & 0.0006 & 2.0934 & 578.19 & 17345.75 & 813495.48 \\
\hline 32 & 960 & 14,126 & 470.87 & 780965 & 0.0006 & 2.0586 & 568.67 & 17060.24 & 830555.73 \\
\hline 33 & 990 & 13,042 & 434.73 & 794007 & 0.0005 & 2.0252 & 559.54 & 16786.18 & 847341.90 \\
\hline
\end{tabular}




\begin{tabular}{|c|c|c|c|c|c|c|c|c|c|}
\hline 34 & 1020 & 12,835 & 427.83 & 806842 & 0.0005 & 1.9932 & 550.76 & 16522.82 & 863864.72 \\
\hline 35 & 1050 & 13,497 & 449.90 & 820339 & 0.0005 & 1.9623 & 542.32 & 16269.49 & 880134.22 \\
\hline 36 & 1080 & 13,073 & 435.77 & 833412 & 0.0005 & 1.9326 & 534.19 & 16025.59 & 896159.81 \\
\hline 37 & 1110 & 13,139 & 437.97 & 846551 & 0.0005 & 1.9040 & 526.35 & 15790.55 & 911950.36 \\
\hline 38 & 1140 & 12,893 & 429.77 & 859444 & 0.0005 & 1.8764 & 518.80 & 15563.86 & 927514.22 \\
\hline 39 & 1170 & 11,840 & 394.67 & 871284 & 0.0005 & 1.8497 & 511.50 & 15345.04 & 942859.26 \\
\hline 40 & 1200 & 12,899 & 429.97 & 884183 & 0.0005 & 1.8239 & 504.45 & 15133.65 & 957992.90 \\
\hline 41 & 1230 & 10,913 & 363.77 & 895096 & 0.0004 & 1.7991 & 497.64 & 14929.29 & 972922.19 \\
\hline 42 & 1260 & 9,747 & 324.90 & 904843 & 0.0004 & 1.7750 & 491.05 & 14731.58 & 987653.77 \\
\hline 43 & 1290 & 13,496 & 449.87 & 918339 & 0.0005 & 1.7517 & 484.67 & 14540.18 & 1002193.95 \\
\hline 44 & 1320 & 12,613 & 420.43 & 930952 & 0.0005 & 1.7291 & 478.49 & 14354.77 & 1016548.72 \\
\hline 45 & 1350 & 13,379 & 445.97 & 944331 & 0.0005 & 1.7072 & 472.50 & 14175.04 & 1030723.77 \\
\hline 46 & 1380 & 11,384 & 379.47 & 955715 & 0.0004 & 1.6860 & 466.69 & 14000.73 & 1044724.49 \\
\hline 47 & 1410 & 9,501 & 316.70 & 965216 & 0.0003 & 1.6653 & 461.05 & 13831.55 & 1058556.05 \\
\hline 48 & 1440 & 5,899 & 196.63 & 971115 & 0.0002 & 1.6453 & 455.58 & 13667.28 & 1072223.33 \\
\hline 49 & 1470 & 4,629 & 154.30 & 975744 & 0.0002 & 1.6259 & 450.26 & 13507.69 & 1085731.02 \\
\hline 50 & 1500 & 17,258 & 575.27 & 993002 & 0.0006 & 1.6070 & 445.09 & 13352.56 & 1099083.58 \\
\hline 51 & 1530 & 32,735 & 1091.17 & 1025737 & 0.0011 & 1.5886 & 440.06 & 13201.70 & 1112285.28 \\
\hline 52 & 1560 & 20,991 & 699.70 & 1046728 & 0.0007 & 1.5707 & 435.16 & 13054.91 & 1125340.18 \\
\hline 53 & 1590 & 18,693 & 623.10 & 1065421 & 0.0006 & 1.5533 & 430.40 & 12912.02 & 1138252.20 \\
\hline 54 & 1620 & 15,782 & 526.07 & 1081203 & 0.0005 & 1.5364 & 425.76 & 12772.86 & 1151025.06 \\
\hline 55 & 1650 & 14,791 & 493.03 & 1095994 & 0.0004 & 1.5199 & 421.24 & 12637.29 & 1163662.35 \\
\hline 56 & 1680 & 12,137 & 404.57 & 1108131 & 0.0004 & 1.5038 & 416.84 & 12505.14 & 1176167.49 \\
\hline 57 & 1710 & 5,851 & 195.03 & 1113982 & 0.0002 & 1.4881 & 412.54 & 12376.29 & 1188543.78 \\
\hline 58 & 1740 & 18,065 & 602.17 & 1132047 & 0.0005 & 1.4728 & 408.35 & 12250.60 & 1200794.37 \\
\hline 59 & 1770 & 17,102 & 570.07 & 1149149 & 0.0005 & 1.4578 & 404.26 & 12127.94 & 1212922.32 \\
\hline 60 & 1800 & 11,324 & 377.47 & 1160473 & 0.0003 & 1.4433 & 400.27 & 12008.21 & 1224930.53 \\
\hline 61 & 1830 & 13,923 & 464.10 & 1174396 & 0.0004 & 1.4290 & 396.38 & 11891.29 & 1236821.82 \\
\hline 62 & 1860 & 12,071 & 402.37 & 1186467 & 0.0003 & 1.4151 & 392.57 & 11777.07 & 1248598.89 \\
\hline 63 & 1890 & 13,287 & 442.90 & 1199754 & 0.0004 & 1.4015 & 388.85 & 11665.46 & 1260264.35 \\
\hline 64 & 1920 & 9,908 & 330.27 & 1209662 & 0.0003 & 1.3882 & 385.21 & 11556.35 & 1271820.70 \\
\hline 65 & 1950 & 14,412 & 480.40 & 1224074 & 0.0004 & 1.3752 & 381.66 & 11449.67 & 1283270.37 \\
\hline 66 & 1980 & 10,569 & 352.30 & 1234643 & 0.0003 & 1.3625 & 378.18 & 11345.32 & 1294615.68 \\
\hline 67 & 2010 & 11,837 & 394.57 & 1246480 & 0.0003 & 1.3501 & 374.77 & 11243.22 & 1305858.90 \\
\hline 68 & 2040 & 14,078 & 469.27 & 1260558 & 0.0004 & 1.3379 & 371.44 & 11143.29 & 1317002.20 \\
\hline 69 & 2070 & 7,603 & 253.43 & 1268161 & 0.0002 & 1.3260 & 368.18 & 11045.47 & 1328047.67 \\
\hline
\end{tabular}


Well No. 1 Calculations (ARP's Model)

\begin{tabular}{|c|c|c|c|c|c|c|c|c|}
\hline & Field & $q^{\wedge}-b$ & q-ARPs & SD-q & ST-q & Gp-ARPs & SD-Gp & ST-Gp \\
\hline (DAYS) & $\begin{array}{c}q \\
\text { (MSCF/DAY) }\end{array}$ & & & & & & & \\
\hline 30 & 1935 & 0.017507 & 1389.494 & 161930.5 & 836650.8 & 84759.82 & $\begin{array}{c}731874680 . \\
3 \\
\end{array}$ & $3.70036 \mathrm{E}+11$ \\
\hline 60 & 1792 & 0.018619 & 1345.419 & 64168.32 & 520590.4 & 125122.4 & 1200804243 & $3.2256 \mathrm{E}+11$ \\
\hline 90 & 1599 & 0.019659 & 1303.46 & 20222.74 & 323138.6 & 164226.2 & 1514674767 & $2.79671 \mathrm{E}+11$ \\
\hline 120 & 1446 & 0.019959 & 1263.483 & 20235.14 & 279332.9 & 202130.7 & 1865059221 & $2.41017 \mathrm{E}+11$ \\
\hline 150 & 1406 & 0.020615 & 1225.363 & 9716.016 & 199558.3 & 238891.6 & $\begin{array}{c}2,129,215,8 \\
55\end{array}$ & $\begin{array}{c}206,274,258,7 \\
47\end{array}$ \\
\hline 180 & 1324 & 0.0224 & 1188.987 & 2889.415 & 66574.07 & 274561.2 & $\begin{array}{c}1,982,994,7 \\
10 \\
\end{array}$ & $\begin{array}{c}175,146,152,1 \\
93\end{array}$ \\
\hline 210 & 1135 & 0.021723 & 1154.247 & 2242.352 & 105226.4 & 309188.6 & 2111534205 & $1.47362 \mathrm{E}+11$ \\
\hline 240 & 1202 & 0.022643 & 1121.045 & 67.43633 & 55516.55 & 342819.9 & 2088953810 & $1.22672 \mathrm{E}+11$ \\
\hline 270 & 1113 & 0.022893 & 1089.292 & 1.227059 & 45448.35 & 375498.7 & 2091992639 & $1.00849 \mathrm{E}+11$ \\
\hline 300 & 1090 & 0.022971 & 1058.903 & 608.2758 & 42581.5 & 407265.8 & 2160223396 & 81681661026 \\
\hline 330 & 1084 & 0.023191 & 1029.801 & 1210.988 & 35113.58 & 438159.8 & 2258357672 & 64977076062 \\
\hline 360 & 1065 & 0.02353 & 1001.912 & 1187.136 & 25329.63 & 468217.2 & 2357668242 & 50556919622 \\
\hline 390 & 1036 & 0.024291 & 975.1693 & 3.600169 & 9970.591 & 497472.3 & 2363199304 & 38256849203 \\
\hline 420 & 977 & 0.024442 & 949.5103 & 269.7159 & 7871.152 & 525957.6 & 2411344167 & 27925174776 \\
\hline 450 & 966 & 0.02528 & 924.8765 & 303.1021 & 915.2347 & 553703.9 & 2360321923 & 19421760847 \\
\hline 480 & 907 & 0.024871 & 901.2133 & 1161.905 & 3374.004 & 580740.3 & 2460730046 & 12617035795 \\
\hline 510 & 935 & 0.025323 & 878.4697 & 682.7923 & 750.0028 & 607094.3 & 2539117327 & 7391096799 \\
\hline 540 & 905 & 0.02726 & 856.5982 & 4542.511 & 7746.433 & 632792.3 & 2339435411 & 3632900076 \\
\hline 570 & 789 & 0.026111 & 835.5541 & 366.567 & 506.8721 & 657858.9 & 2395328040 & 1239527354 \\
\hline 600 & 855 & 0.027246 & 815.2957 & 643.2497 & 7617.884 & 682317.8 & 2321429729 & 115520598.8 \\
\hline 630 & 790 & 0.027572 & 795.784 & 532.8694 & 10923.14 & 706191.3 & 2255176503 & 172277909.8 \\
\hline 660 & 773 & 0.027879 & 776.9822 & 397.9556 & 14443.35 & 729500.8 & 2198694041 & 1327504321 \\
\hline 690 & 757 & 0.036836 & 758.8558 & 94221.88 & 180891.8 & 752266.4 & 1419899887 & 3504711950 \\
\hline 720 & 452 & 0.026633 & 741.3726 & 6816.271 & 2838.81 & 774507.6 & 1612695511 & 6632764561 \\
\hline 750 & 824 & 0.027579 & 724.5021 & 2287.828 & 10999.92 & 796242.7 & 1730004086 & 10645462141 \\
\hline 780 & 772 & 0.028108 & 708.2156 & 1402.584 & 17304.65 & 817489.1 & 1824729322 & 15481161591 \\
\hline 810 & 746 & 0.029981 & 692.4861 & 947.7848 & 46446.21 & 838263.7 & 1746677181 & 21082430019 \\
\hline 840 & 662 & 0.029958 & 677.2883 & 214.7672 & 46044.79 & 858582.4 & 1710121808 & 27395727538 \\
\hline 870 & 663 & 0.030132 & 662.5981 & 49.44056 & 49127.46 & 878460.3 & 1692719878 & 34371116764 \\
\hline 900 & 656 & 0.031485 & 648.3929 & 1938.306 & 74445.57 & 897912.1 & 1585783029 & 41961996521 \\
\hline 930 & 604 & 0.044155 & 634.6513 & 97084.97 & 307079.1 & 916951.6 & $\begin{array}{c}928686198 . \\
1\end{array}$ & 50124857507 \\
\hline 960 & 323 & 0.031173 & 621.3531 & 33.09819 & 68441.79 & 935592.2 & 918196661 & 58819057915 \\
\hline 990 & 616 & 0.025709 & 608.4792 & 73524.54 & 5.854039 & 953846.6 & 1477355529 & 68006617192 \\
\hline
\end{tabular}




\begin{tabular}{|c|c|c|c|c|c|c|c|c|}
\hline 1020 & 880 & 0.030176 & 596.0116 & 3339.5 & 49913.74 & 971727 & 1613631711 & 77652026309 \\
\hline 1050 & 654 & 0.03098 & 583.9331 & 1505.462 & 64760.32 & 989245 & 1708503140 & 87722073088 \\
\hline 1080 & 623 & 0.03219 & 572.2274 & 61.45428 & 88296.43 & 1006412 & 1728000192 & 98185681232 \\
\hline 1110 & 580 & 0.031184 & 560.8792 & 2950.747 & 68651.24 & 1023238 & 1866140222 & $1.09014 \mathrm{E}+11$ \\
\hline 1140 & 615 & 0.031803 & 549.874 & 1877.146 & 80663.85 & 1039734 & 1980127766 & $1.20179 \mathrm{E}+11$ \\
\hline 1170 & 593 & 0.031781 & 539.1977 & 2999.635 & 80228.95 & 1055910 & 2129055945 & $1.31656 \mathrm{E}+11$ \\
\hline 1200 & 594 & 0.031813 & 528.8374 & 4099.741 & 80853.3 & 1071775 & 2310010722 & $1.43421 \mathrm{E}+11$ \\
\hline 1230 & 593 & 0.032403 & 518.7806 & 2943.364 & 92525.77 & 1087339 & 2469111434 & $1.55451 \mathrm{E}+11$ \\
\hline 1260 & 573 & 0.032143 & 509.0152 & 5273.394 & 87367.82 & 1102609 & 2690361880 & $1.67726 \mathrm{E}+11$ \\
\hline 1290 & 582 & 0.034922 & 499.5301 & 0.485439 & 143171.8 & 1117595 & 2688193993 & $1.80225 \mathrm{E}+11$ \\
\hline 1320 & 499 & 0.034246 & 490.3144 & 724.6279 & 129586 & 1132305 & 2772587337 & $1.92931 \mathrm{E}+11$ \\
\hline 1350 & 517 & 0.034836 & 481.358 & 391.0633 & 141436.5 & 1146745 & 2835415893 & $2.05825 \mathrm{E}+11$ \\
\hline 1380 & 501 & 0.03427 & 472.6511 & 1928.578 & 130066.4 & 1160925 & 2977458242 & $2.18892 E+11$ \\
\hline 1410 & 517 & 0.03419 & 464.1844 & 2982.868 & 128460.5 & 1174850 & 3158952526 & $2.32116 \mathrm{E}+11$ \\
\hline 1440 & 519 & 0.034595 & 455.949 & 2667.83 & 136614.4 & 1188529 & 3335534998 & $2.45484 \mathrm{E}+11$ \\
\hline 1470 & 508 & 0.035124 & 447.9364 & 2079.077 & 147210.7 & 1201967 & 3495410613 & $2.5898 \mathrm{E}+11$ \\
\hline 1500 & 494 & 0.035855 & 440.1387 & 1219.962 & 161722.3 & 1215171 & 3620409220 & $2.72594 \mathrm{E}+11$ \\
\hline 1530 & 475 & 0.035052 & 432.5481 & 3954.555 & 145756.3 & 1228148 & 3850996048 & $2.86313 \mathrm{E}+11$ \\
\hline 1560 & 495 & 0.03402 & 425.1572 & 9690.988 & 125042.7 & 1240902 & 4226258299 & $3.00125 \mathrm{E}+11$ \\
\hline 1590 & 524 & 0.03718 & 417.959 & 688.5902 & 187501 & 1253441 & 4329233179 & $3.1402 \mathrm{E}+11$ \\
\hline 1620 & 444 & 0.034414 & 410.9468 & 10326.6 & 132967.5 & 1265769 & 4739703557 & $3.27989 \mathrm{E}+11$ \\
\hline 1650 & 513 & 0.034524 & 404.1141 & 11113.21 & 135188.9 & 1277893 & 5185163774 & $3.42023 \mathrm{E}+11$ \\
\hline 1680 & 510 & 0.033558 & 397.4549 & 19482.15 & 115722.8 & 1289817 & 5805744433 & $3.56111 \mathrm{E}+11$ \\
\hline 1710 & 537 & 0.036271 & 390.9631 & 5486.402 & 169892.8 & 1301545 & 6149311099 & $3.70247 E+11$ \\
\hline 1740 & 465 & 0.036237 & 384.6332 & 6593.466 & 169233.9 & 1313084 & 6537296238 & $3.84423 \mathrm{E}+11$ \\
\hline 1770 & 466 & 0.03797 & 378.4597 & 2378.865 & 202482.4 & 1324438 & 6776048379 & $3.98631 \mathrm{E}+11$ \\
\hline 1800 & 427 & 0.03833 & 372.4376 & 2246.36 & 209196.9 & 1335611 & 7012158116 & $4.12865 \mathrm{E}+11$ \\
\hline 1830 & 420 & 0.037748 & 366.5617 & 4269.094 & 198304.4 & 1346608 & 7344280695 & $4.27118 \mathrm{E}+11$ \\
\hline 1860 & 432 & 0.038467 & 360.8274 & 3162.856 & 211735.4 & 1357433 & 7636305474 & $4.41384 \mathrm{E}+11$ \\
\hline 1890 & 417 & 0.037944 & 355.2301 & 5261.556 & 202002.7 & 1368090 & 8021361608 & $4.55658 \mathrm{E}+11$ \\
\hline 1920 & 428 & 0.038049 & 349.7654 & 5750.891 & 203955 & 1378583 & 8434051721 & $4.69934 \mathrm{E}+11$ \\
\hline 1950 & 426 & 0.04032 & 344.4291 & 1431.685 & 244972.7 & 1388916 & 8643834009 & $4.84207 \mathrm{E}+11$ \\
\hline 1980 & 382 & 0.037757 & 339.2171 & 8553.088 & 198482.6 & 1399092 & 9167432255 & $4.98473 \mathrm{E}+11$ \\
\hline 2010 & 432 & 0.037442 & 334.1256 & 10887.06 & 192499.1 & 1409116 & 9776649390 & $5.12728 \mathrm{E}+11$ \\
\hline 2040 & 438 & 0.037392 & 329.1509 & 12184.29 & 191564.2 & 1418991 & $\begin{array}{c}1044247212 \\
9 \\
\end{array}$ & $5.26967 \mathrm{E}+11$ \\
\hline 2070 & 440 & 0.037765 & 324.2893 & 11501.29 & 198631.1 & 1428719 & $\begin{array}{c}1111036935 \\
3 \\
\end{array}$ & $5.41186 \mathrm{E}+11$ \\
\hline 2100 & 432 & 0.037202 & 319.5374 & 15416.36 & 187934.2 & 1438305 & $\begin{array}{c}1190949121 \\
0 \\
\end{array}$ & $5.55382 \mathrm{E}+11$ \\
\hline 2130 & 444 & 0.037691 & 314.8919 & 13973.16 & 197237.1 & 1447752 & $\begin{array}{c}1269607451 \\
9\end{array}$ & $5.69551 \mathrm{E}+11$ \\
\hline
\end{tabular}




\begin{tabular}{|c|c|c|c|c|c|c|c|c|}
\hline 2160 & 433 & 0.038374 & 310.3496 & 11790.43 & 210021 & 1457063 & $\begin{array}{c}1344077845 \\
9\end{array}$ & $5.83691 \mathrm{E}+11$ \\
\hline 2190 & 419 & 0.037862 & 305.9074 & 15275.13 & 200447.7 & 1466240 & $\begin{array}{c}1431424385 \\
1\end{array}$ & $5.97798 \mathrm{E}+11$ \\
\hline 2220 & 430 & 0.038514 & 301.5624 & 13126.5 & 212595.2 & 1475287 & $\begin{array}{c}1514850848 \\
7 \\
\end{array}$ & $6.1187 \mathrm{E}+11$ \\
\hline 2250 & 416 & 0.038469 & 297.3117 & 14333.27 & 211766.1 & 1484206 & $\begin{array}{c}1604552352 \\
7\end{array}$ & $6.25903 E+11$ \\
\hline 2280 & 417 & 0.039609 & 293.1526 & 10386.48 & 232465.9 & 1493001 & $\begin{array}{c}1682944443 \\
3\end{array}$ & $6.39896 \mathrm{E}+11$ \\
\hline 2310 & 395 & 0.043269 & 289.0824 & 2148.41 & 293526.1 & 1501673 & $\begin{array}{c}1719215985 \\
9\end{array}$ & $6.53846 \mathrm{E}+11$ \\
\hline 2340 & 335 & 0.039345 & 285.0986 & 13202.34 & 227733 & 1510226 & $\begin{array}{c}1810798665 \\
8\end{array}$ & $6.67751 \mathrm{E}+11$ \\
\hline 2370 & 400 & 0.040318 & 281.1987 & 10221.47 & 244939.7 & 1518662 & $\begin{array}{c}1893347341 \\
4 \\
\end{array}$ & $6.81609 \mathrm{E}+11$ \\
\hline 2400 & 382 & 0.039599 & 277.3804 & 13897.18 & 232273.1 & 1526983 & $\begin{array}{c}1991924291 \\
3\end{array}$ & $6.95419 \mathrm{E}+11$ \\
\hline 2430 & 395 & 0.039829 & 273.6413 & 13780.89 & 236371.5 & 1535193 & $\begin{array}{c}2092573721 \\
8\end{array}$ & $7.09178 \mathrm{E}+11$ \\
\hline 2460 & 391 & 0.039355 & 269.9793 & 16853.42 & 227924 & 1543292 & $\begin{array}{c}2206767638 \\
3\end{array}$ & $7.22885 E+11$ \\
\hline 2490 & 400 & 0.039958 & 266.3922 & 14959.2 & 238645.8 & 1551284 & $\begin{array}{c}2317128343 \\
1\end{array}$ & $7.36538 \mathrm{E}+11$ \\
\hline 2520 & 389 & 0.040797 & 262.878 & 12355.52 & 253190.6 & 1559170 & $\begin{array}{c}2419761534 \\
7 \\
\end{array}$ & $7.50137 \mathrm{E}+11$ \\
\hline 2550 & 374 & 0.040003 & 259.4346 & 16503.35 & 239428 & 1566953 & $\begin{array}{c}2541148043 \\
9\end{array}$ & $7.63679 E+11$ \\
\hline 2580 & 388 & 0.041761 & 256.0603 & 10432.52 & 269375.3 & 1574635 & $\begin{array}{c}2639779387 \\
9 \\
\end{array}$ & $7.77164 \mathrm{E}+11$ \\
\hline 2610 & 358 & 0.04147 & 252.7531 & 12125.01 & 264553 & 1582218 & $\begin{array}{c}2748214221 \\
0\end{array}$ & $7.90591 E+11$ \\
\hline 2640 & 363 & 0.028799 & 249.5111 & 214698.3 & 27009.99 & 1589703 & $\begin{array}{c}3228420282 \\
7 \\
\end{array}$ & $8.03958 \mathrm{E}+11$ \\
\hline 2670 & 713 & 0.029112 & 246.3328 & 204666.2 & 31855.28 & 1597093 & $\begin{array}{c}3734558897 \\
1\end{array}$ & $8.17265 E+11$ \\
\hline 2700 & 699 & 0.027824 & 243.2164 & 266858.6 & 13786.01 & 1604389 & $\begin{array}{c}4357555027 \\
9 \\
\end{array}$ & $8.30511 \mathrm{E}+11$ \\
\hline 2730 & 760 & 0.02868 & 240.1603 & 228649.4 & 25243.01 & 1611594 & $\begin{array}{c}4977038188 \\
4\end{array}$ & $8.43694 E+11$ \\
\hline 2760 & 718 & 0.0278 & 237.163 & 274405.2 & 13505.65 & 1618709 & $\begin{array}{c}5702920136 \\
4\end{array}$ & $8.56816 E+11$ \\
\hline 2790 & 761 & 0.041913 & 234.2229 & 14781 & 271872.4 & 1625736 & $\begin{array}{c}5878451890 \\
0 \\
\end{array}$ & $8.69873 E+11$ \\
\hline 2820 & 356 & 0.04228 & 231.3385 & 14104.29 & 277849 & 1632676 & $\begin{array}{c}6052487334 \\
4\end{array}$ & $8.82867 \mathrm{E}+11$ \\
\hline 2850 & 350 & 0.043158 & 228.5085 & 11777.65 & 291795 & 1639531 & $\begin{array}{c}6213741770 \\
4\end{array}$ & $8.95797 \mathrm{E}+11$ \\
\hline 2880 & 337 & 0.043158 & 225.7314 & 12388.12 & 291795 & 1646303 & $\begin{array}{c}6381324621 \\
5 \\
\end{array}$ & $9.08661 E+11$ \\
\hline
\end{tabular}




\begin{tabular}{|c|c|c|c|c|c|c|c|c|}
\hline 2910 & 337 & 0.041337 & 223.006 & 20171.77 & 262328.9 & 1652993 & $\begin{array}{c}6598407689 \\
9 \\
\end{array}$ & $9.21461 \mathrm{E}+11$ \\
\hline 2940 & 365 & 0.042601 & 220.3309 & 15600.62 & 283003.2 & 1659603 & $\begin{array}{c}6792316631 \\
2 \\
\end{array}$ & $9.34195 \mathrm{E}+11$ \\
\hline 2970 & 345 & 0.043357 & 217.7049 & 13563.34 & 294900.2 & 1666134 & $\begin{array}{c}6975651425 \\
7\end{array}$ & $9.46862 E+11$ \\
\hline 3000 & 334 & 0.044242 & 215.1268 & 11400.51 & 308373.4 & 1672588 & $\begin{array}{c}7145879605 \\
7 \\
\end{array}$ & $9.59464 E+11$ \\
\hline 3030 & 322 & 0.04523 & 212.5954 & 9293.839 & 322866.9 & 1678966 & $\begin{array}{c}7301339971 \\
5 \\
\end{array}$ & $9.71999 \mathrm{E}+11$ \\
\hline 3060 & 309 & 0.043273 & 210.1096 & 15689.32 & 293598.3 & 1685269 & $\begin{array}{c}7505826148 \\
2\end{array}$ & $9.84468 \mathrm{E}+11$ \\
\hline 3090 & 335 & 0.049986 & 207.6683 & 2410.647 & 384954.7 & 1691499 & $\begin{array}{c}7586751235 \\
4\end{array}$ & $9.9687 E+11$ \\
\hline 3120 & 257 & 0.05294 & 205.2704 & 655.1675 & 417764.6 & 1697658 & $\begin{array}{c}7629111658 \\
6\end{array}$ & $1.0092 \mathrm{E}+12$ \\
\hline 3150 & 231 & 0.043086 & 202.9149 & 18266 & 290679.7 & 1703745 & $\begin{array}{c}7854735972 \\
3 \\
\end{array}$ & $1.02147 \mathrm{E}+12$ \\
\hline 3180 & 338 & 0.085286 & 200.6007 & 11053.17 & 611128.6 & 1709763 & $\begin{array}{c}7678939598 \\
2 \\
\end{array}$ & $1.03367 \mathrm{E}+12$ \\
\hline 3210 & 95 & 0.065885 & 198.327 & 1967.837 & 523086.4 & 1715713 & $\begin{array}{c}7605360853 \\
6 \\
\end{array}$ & $1.04581 \mathrm{E}+12$ \\
\hline 3240 & 154 & 0.043441 & 196.0927 & 18734.49 & 296205 & 1721596 & $\begin{array}{c}7833528143 \\
2\end{array}$ & $1.05787 \mathrm{E}+12$ \\
\hline 3270 & 333 & 0.047686 & 193.8969 & 7442.476 & 356465.3 & 1727412 & $\begin{array}{c}7979071375 \\
9\end{array}$ & $1.06987 \mathrm{E}+12$ \\
\hline 3300 & 280 & 0.057104 & 191.7387 & 79.70803 & 457716.1 & 1733165 & $\begin{array}{c}7994209926 \\
7\end{array}$ & $1.08181 \mathrm{E}+12$ \\
\hline 3330 & 201 & & & & & & & \\
\hline
\end{tabular}

